\title{
Active Control of Tip Clearance Flow in Axial Compressors
}

by

\author{
Jinwoo Bae \\ B.S., Aerospace Engineering, Seoul National University, Korea (1995) \\ S.M., Aeronautics and Astronautics, Massachusetts Institute of Technology (1998) \\ Submitted to the Department of Aeronautics and Astronautics \\ in Partial Fulfillment of the Requirements for the Degree of

\section{Doctor of Philosophy} \\ at the \\ Massachusetts Institute of Technology
}

June 2001

(C) 2001 Massachusetts Institute of Technology. All rights reserved.

Signature of Author

Department of Aeronautics and Astronautics May 8, 2001

Certified by

Kenneth S. Breuer, Ph.D.

Visiting Associate Professor of Aeronautics and Astronautics

7 Associate Professor of Division of Engineering, Brown University

Thesis Supervisor

Certified by

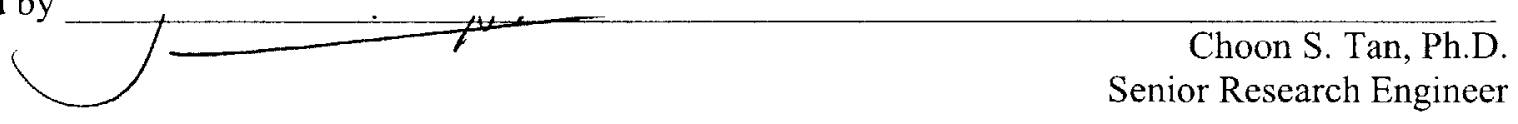

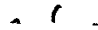

Certified by

Edward M. Greitzer, Ph.D

H. Nelson Slater Professor of Aeronautics and Astronautics

Accepted by

Wallace E. Vander Velde, Sc.D

MASSACHUSETTS INSTITUTE

OF TECHNOLOGY

SEP 112001 


\title{
Active Control of Tip Clearance Flow in Axial Compressors
}

\author{
By \\ Jinwoo Bae \\ Submitted to the Department of Aeronautics and Astronautics \\ on May 8, 2001 in Partial Fulfillment of the \\ Requirements for the Degree of \\ Doctor of Philosophy
}

\begin{abstract}
Control of compressor tip clearance flows is explored in a linear cascade using three types of fluidic actuators; Normal Synthetic Jet (NSJ; unsteady jet normal to the mean flow with zero net mass flux), Directed Synthetic Jet (DSJ; injection roughly aligned with the mean flow), and Steady Directed Jet (SDJ), mounted on the casing wall. The objective is to affect the following measures: (1) reduction of tip leakage flow rate, (2) mixing enhancement between tip leakage and core flow, and (3) increase in streamwise momentum of the flow in the endwall region. The measurements show that the NSJ provides mixing enhancement only, or both mixing enhancement and leakage flow reduction, depending on its pitchwise location. The DSJ and SDJ actuators provide streamwise momentum enhancement with a consequent reduction of clearance-related blockage.

The blockage reduction associated with the use of NSJ is sensitive to actuator frequency, whereas that with the use of DSJ is not. For a given actuation amplitude, DSJ and SDJ are about twice as effective as NSJ in reducing clearance-related blockage. Further the DSJ and SDJ can eliminate clearance-related blockage with a time-averaged momentum flux roughly $16 \%$ of the momentum flux of the leakage flow. However, achieving overall gain in efficiency appears to be hard; the decrease in loss is only about $30 \%$ of the expended flow power from the present SDJ actuator, which is the best among the actuators considered. Guidelines for improving the efficiency of the directed jet actuation are presented.

Time-resolved measurements show periodic unsteadiness of the tip clearance vortex with the peak frequency corresponding to the optimum condition for blockage reduction with the NSJ. A physical explanation of the source of the observed periodic unsteadiness is suggested based on trailing vortex instability theory. Observations of the time scale for the unsteadiness from different compressor geometries and flow conditions are shown to scale with a reduced frequency based on convective time through the blade passage.
\end{abstract}

Thesis Supervisor: Kenneth S. Breuer, Ph.D.

Title: Visiting Associate Professor of Aeronautics and Astronautics Associate Professor of Division of Engineering, Brown University 


\section{ACKNOWLEDGEMENT}

I would like to express my deep gratitude to those who made it possible for me to make this accomplishment. First, I would like to thank my thesis committee members: my advisor, Prof. Kenny Breuer for his support, guidance, and encouragement throughout this project, Dr. C. S. Tan for his support and technical advice, Prof. E. M. Greitzer for many helpful comments and questions, and Prof. M. A. Schmidt for guiding me though my minor field, MEMS. I also owe thanks to the following individuals for their insightful comments and help during the project: Prof. F. E. Marble, Prof. N. A. Cumpsty, Prof. A. H. Epstein, Prof. E. Covert, Prof. M. Drela, Dr. J. D. Paduano, Dr. G. Guenette, Dr. T. Beutner, and Prof. Waitz.

The cascade tunnel test section could not have been built without the support and assistance of Bill Ames, Viktor Dubrowski, Jimmy Letendre, Paul Warren, and Richard Perdichizzi. Their assistance and friendliness are appreciated. I also would like to thank Holly Aderson, Lori Martinez, and Marie Stuppard for their administrative support, and Diana Park for her help with graphics. Many fellow students helped me carrying out this research and I would especially like to thank Duc Vo, Eugene Kang, Shangfang Liao, Rory Keogh, Yifang Gong, Zolti Spakovszky, Luc Frechette, Patrick Shum, Asif Khalak, Chris Spadaccini, Taek Jin Choi, Ashish Verma and Boris Sirakov. I also thank my friends of GTL and MIT Korean Graduate Students Association for sharing the fun at MIT.

I am indebted to my parents, sister and parents-in-law for their continued support. Thanks to my wife, Jung Yoon Ok not only for her loving encouragement but also for helping me with her jeweler's talent in casting the blades and fixing some of the probes. This thesis is dedicated to her.

This work was funded by DARPA/AFOSR under Grant F49620-97-1-0526 monitored by Dr. W. Tang and Dr. T. J. Beutner whose support is gratefully acknowledged. I am especially grateful to Dr. Beutner for his patience and additional support to complete this research.

I would also like to thank Dean B.E. Staton for her financial support for the last semester of the endeavor. 


\section{CONTENTS}

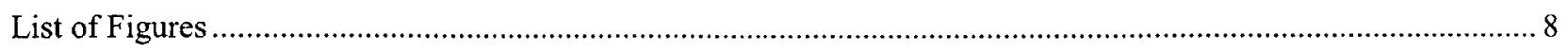

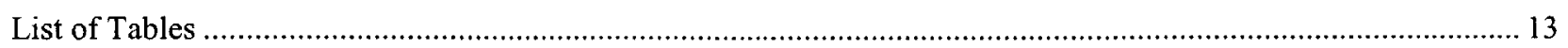

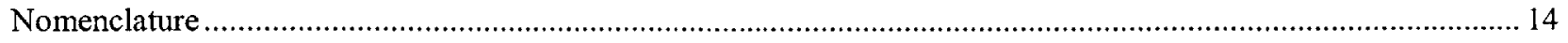

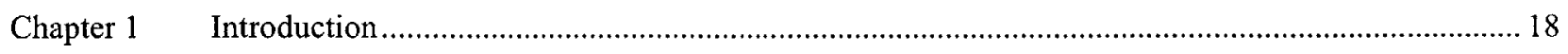

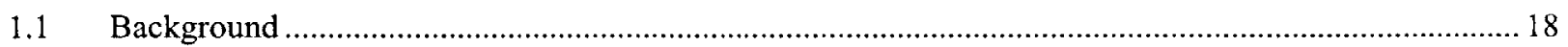

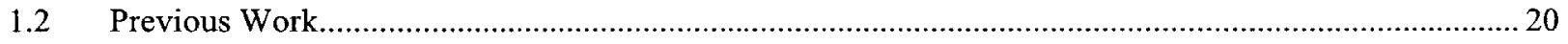

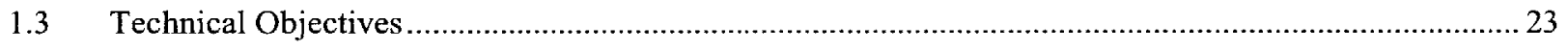

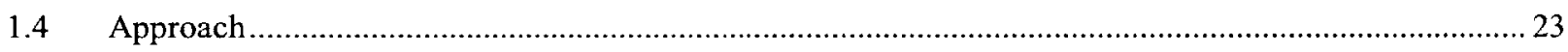

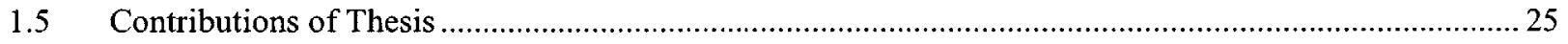

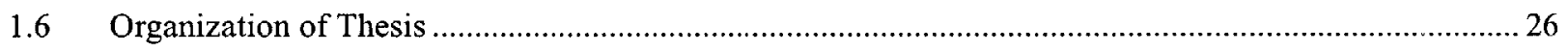

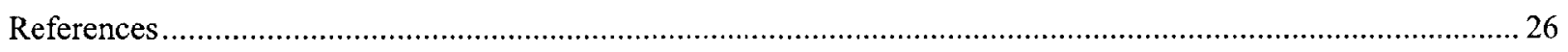

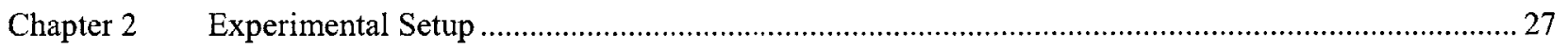

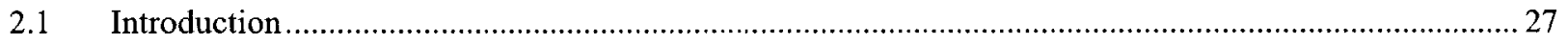

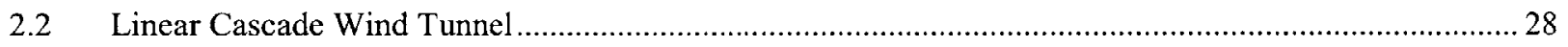

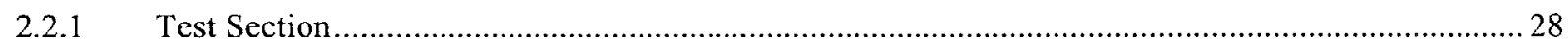

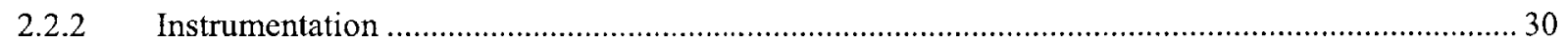

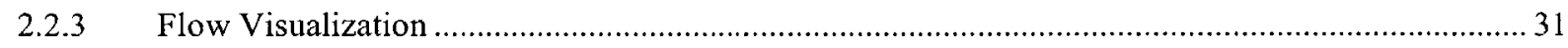

$2.3 \quad$ Actuator

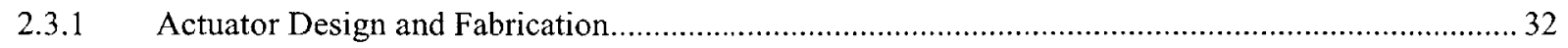

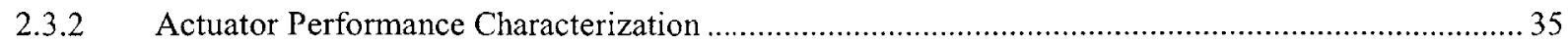

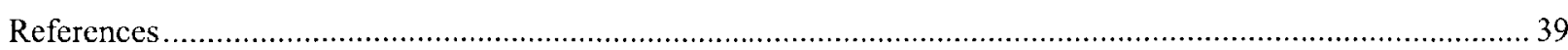

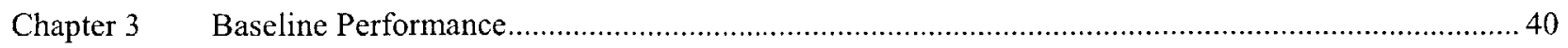

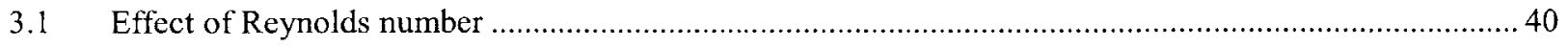

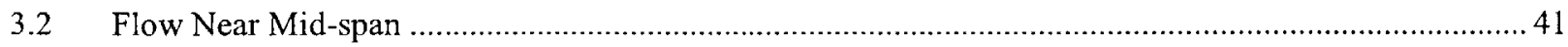

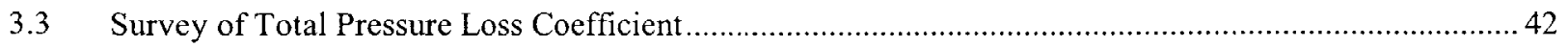

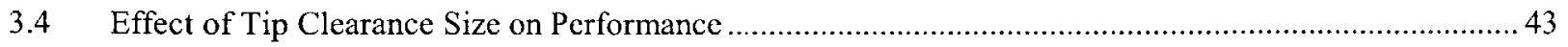




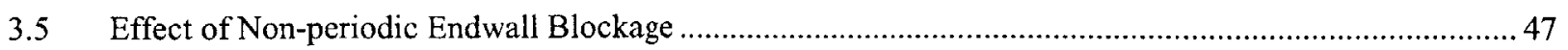

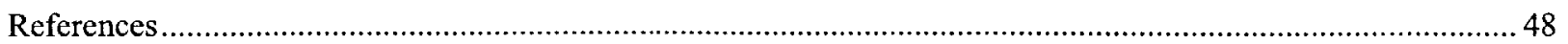

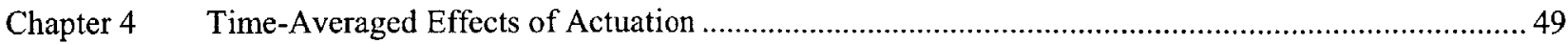

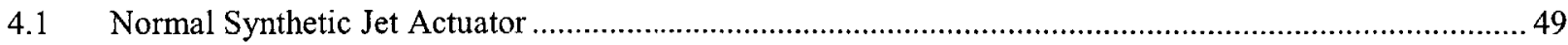

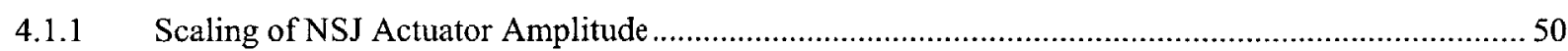

4.1.2 Dependence on Forcing Frequency of NSJ ................................................................................. 52

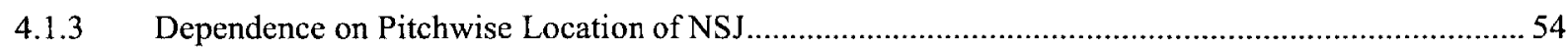

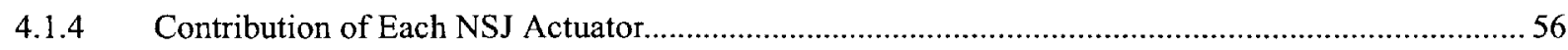

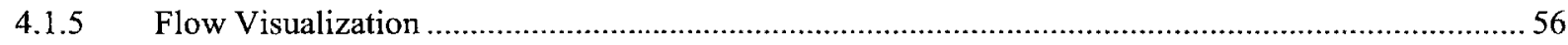

4.1.6 Bulk Flow Process Associated with NSJ Actuation....................................................................... 58

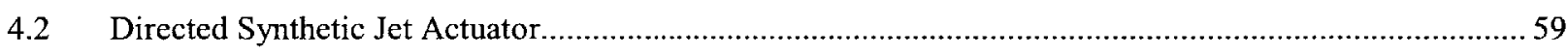

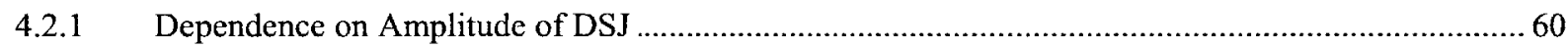

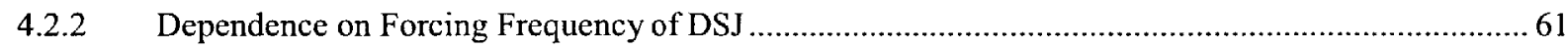

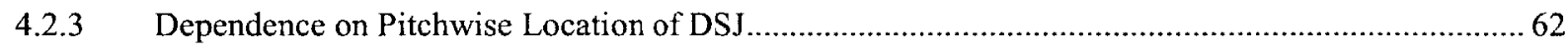

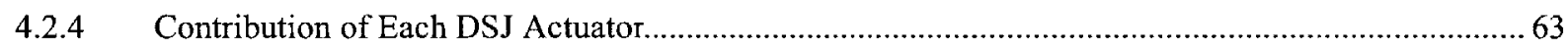

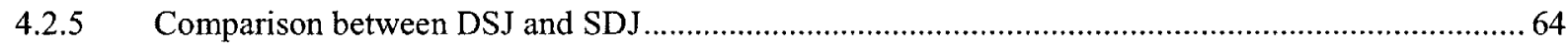

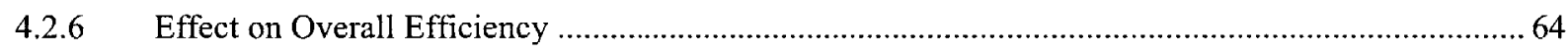

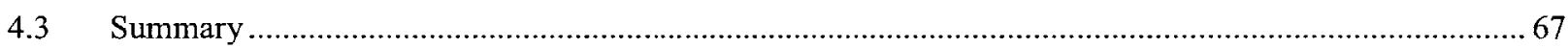

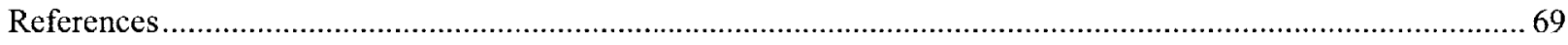

Chapter 5 Unsteadiness in Tip Clearance Vortex ................................................................................... 70

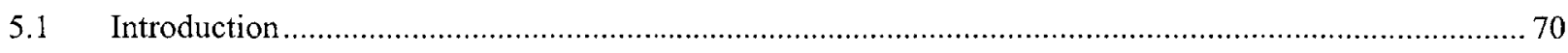

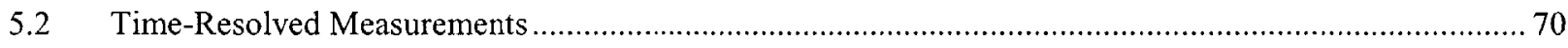

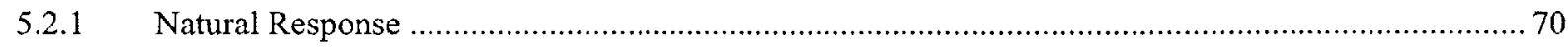

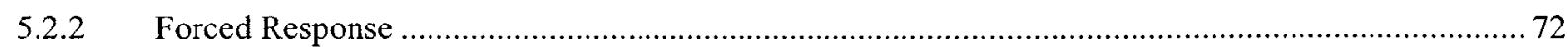

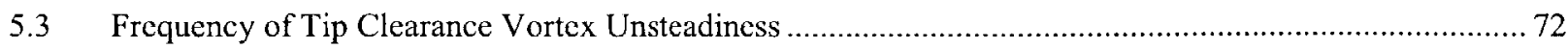

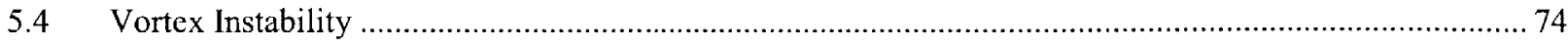

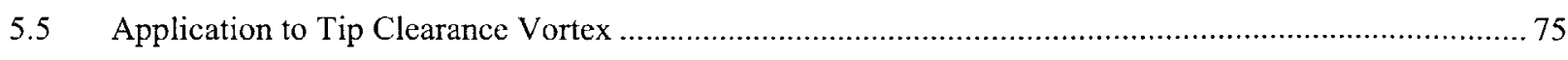

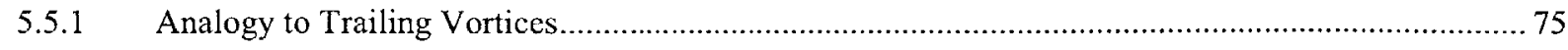




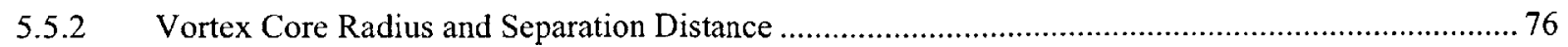

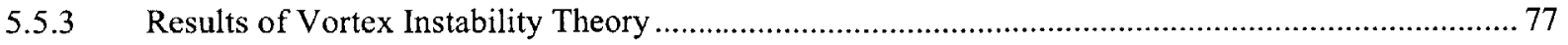

5.5.4 Relevance of Blade Chord: A Thought Experiment.................................................................... 79

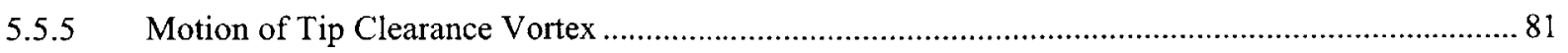

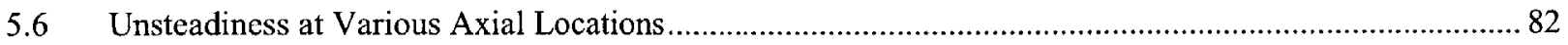

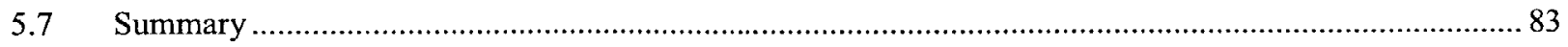

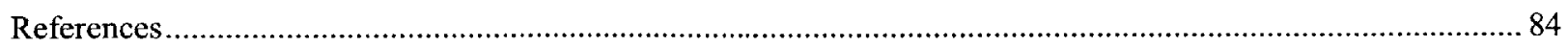

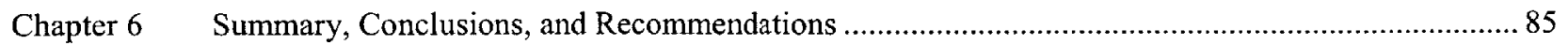

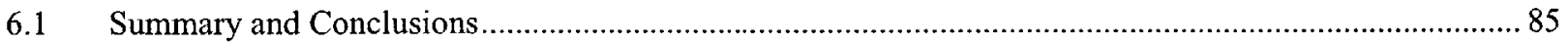

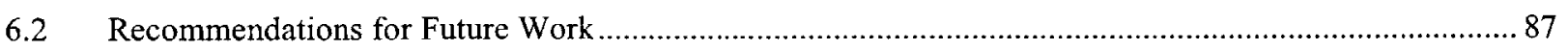

Appendix A $\quad$ Two-dimensional Leakage Jet Experiment ……........................................................................ 88

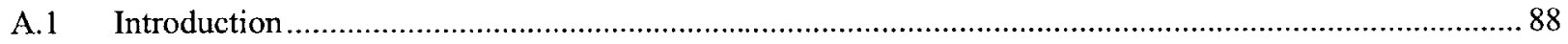

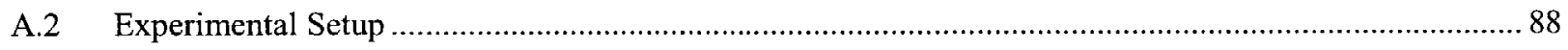

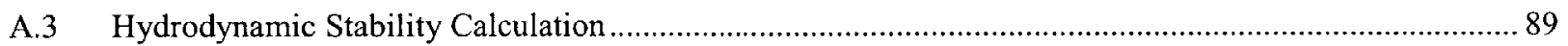

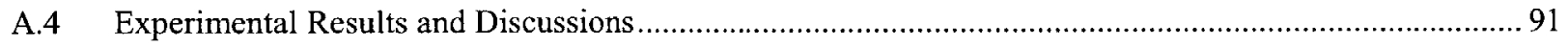

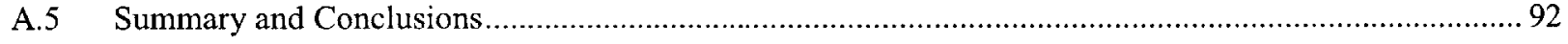

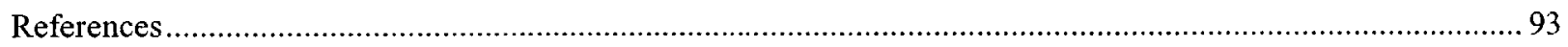




\section{LIST OF FIGURES}

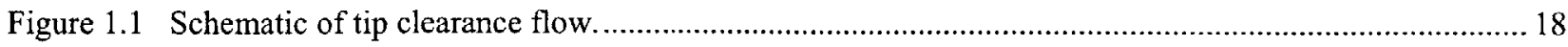

Figure 1.2 Effect of increased tip clearance on overall compressor performance (Wisler [1.1]).......................... 19

Figure 1.3 Effect of tip clearance on peak pressure rise (Smith [1.2]) .........................................................22

Figure 1.4 Relation between rotor maximum efficiency and stall margin improvement for various casing treatment

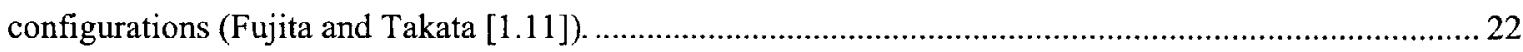

Figure 1.5 Illustration of tip leakage flow rate reduction scheme

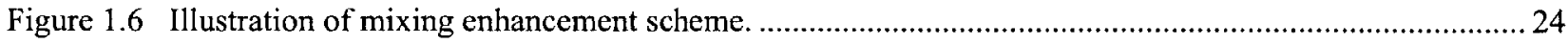

Figure 1.7 Illustration of streamwise momentum injection scheme................................................................2 25

Figure 2.1 Effect of tip clearance on endwall flow structure. Trailing edge total pressure loss coefficient $\omega$ contours of cantilevered stator with (a) moving wall, skewed boundary layer, and clearance; (b) stationary wall, skewed boundary layer, and clearance; (c) stationary wall, collateral boundary layer, and clearance; (d) stationary wall, collateral boundary layer, and no clearance. CFD simulation (Khalid [2.5]) …................28

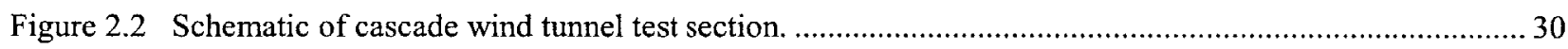

Figure 2.3 Schematic of synthetic jet actuator used in cascade rig. Configuration of Normal Synthetic Jet actuator with slit. 32

Figure 2.4 Schematic of DSJ actuator mounted on casing wall. 33

Figure 2.5 Schematic of Directed Synthetic Jet (DSJ) actuator mounted on casing wall showing the direction of the jet. 34

Figure 2.6 Amplitude/frequency response of NSJ actuator: (a) comparing pre- and post-calibration on linear scale abscissa; (b) calibration taken over larger frequency range shown on log scale abscissa. Actuator slit size $d$ $=0.25 \mathrm{~mm} . \mathrm{Vpp}$ is peak-to-peak driving voltage. Data of two different membranes. .36

Figure 2.7 Comparison between measured and simulated impulse responses of voice coil and membrane system. 37

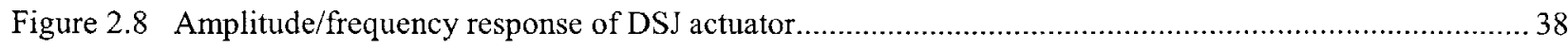

Figure 2.9 Time-trace of DSJ: (a) velocity; (b) momentum. Ensemble-average of 20 cycles............................... 38

Figure 2.10 Calibration of Steady Directed Jet (SDJ) actuator.................................................................... 39

Figure 3.1 Contours of total pressure loss coefficient with various Reynolds numbers. $3 \% C$ tip clearance. ........... 41 
Figure 3.2 Reynolds number dependence of flow blockage. All quantitative measurements are done with $R e_{C}$ above $100,000$. .41

Figure 3.3 Mid-span $C_{P}$ distribution. Comaprison between measurements on center blade and CFD prediction using MISES [3.1]. .42

Figure 3.4 Contours of total pressure loss coefficient, $\omega$ measured $5 \% \mathrm{C}$ downstream of trailing edge plane.......... 43

Figure 3.5 Endwall blockage measured $5 \% \mathrm{C}$ downstream of trailing edge plane vs. tip clearance size. 44

Figure 3.6 Static pressure measured 5\%C downstream of trailing edge plane using six pressure taps on endwall. . 45

Figure 3.7 Mass-averaged endwall total pressure loss coefficient vs. tip clearance size. .46

Figure 3.8 Stream thrust-averaged total pressure loss coefficient vs. tip clearance size. .46

Figure 3.9 Measurements with non-periodic tip clearances compared with measurements with uniform tip clearances: (a) endwall blockage; (b) mass-averaged endwall loss. .48

Figure 4.1 Contours of $\omega$ measured $5 \% C$ downstream of trailing edge plane: (a) baseline without actuation, $\tau=$ $3 \% C$; (b) NSJ directly over blade tip (at $0 \%$ pitch), $C_{\mu, \tau}=0.88, F_{C}{ }^{+}=1.0 ;$ (c) NSJ over vortex core (at $25 \%$ pitch), $C_{\mu, \tau}=0.88, F_{C}^{+}=1.0$. Arrows indicate pitchwise locations of NSJ slits. 49

Figure 4.2 Exit static pressure with and without NSJ actuation. $\triangleleft$ : NSJ directly over blade tip (at $0 \%$ pitch), $C_{\mu, \tau}=$

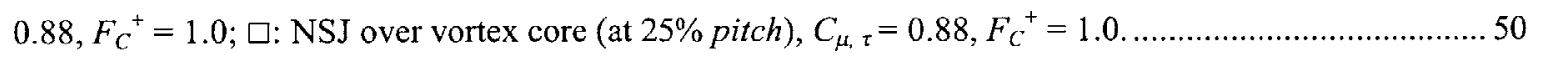

Figure 4.3 Magnitude of leakage jet velocity leaving tip clearance. 51

Figure 4.4 Tip clearance-related blockage vs. NSJ actuator amplitude. Actuator near vortex core. $F_{C}{ }^{+}=1.0 \ldots \ldots \ldots .51$

Figure 4.5 Contours of $\omega$ measured $5 \% \mathrm{C}$ downstream of trailing edge plane: (a) baseline without actuation, $\tau=$ $2 \% C$; (b) NSJ over vortex core (at $25 \%$ pitch), $C_{\mu, \tau}=0.44,{F_{C}}^{+}=1.0$; (c) NSJ over vortex core, $C_{\mu, \tau}=$

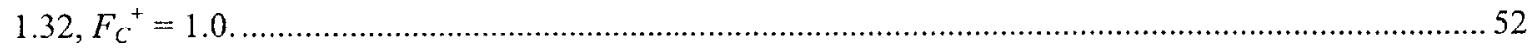

Figure 4.6 Endwall blockage vs. $C_{\mu, \tau}$ of NSJ direcly over blade tip. Data sets taken for two upstream velocities. $\tau=$ $3 \% C, F_{C}{ }^{+}=1.0$.

Figure 4.7 Frequency dependence of blockage reduction with NSJ actuator over vortex core: (a) using clearance size $\tau$, (b) using blade chord $C$ as length scale. 53

Figure 4.8 Frequency dependence of blockage reduction with NSJ actuator over blade tip: (a) using clearance size $\tau$, (b) using blade chord $C$ as length scale. 54 
Figure 4.9 Endwall blockage vs. pitchwise location of NSJ actuator. $\tau / C=3 \%, C_{\mu, \tau}=0.88$ and $F_{C}{ }^{+}=1.0 \ldots \ldots \ldots . .55$

Figure 4.10 Endwall total pressure loss coefficient vs. pitchwise location of NSJ actuator. $\tau / C=3 \%, C_{\mu, \tau}=0.88$ and $F_{C}{ }^{+}=1.0$ : (a) mass-averaged $\omega$, (b) stream thrust-averaged (or fully mixed-out) $\omega$.

Figure 4.11 Contribution of each NSJ actuator placed directly over blade tip. Amplitude is total $C_{\mu, \tau}$ from all working actuators. All data taken with $F_{C}{ }^{+}=1.0$ and $\tau / C=3 \%$. 56

Figure 4.12 Images from flow visualization and computer-processed intensity contour lines: (a) baseline without actuation $(\tau=3 \% C)$; (b) with NSJ actuation over blade tip $\left(F_{C}^{+}=1.0\right.$ and $\left.C_{\mu, \tau}=0.88\right)$. .57

Figure 4.13 Average magnitude of gradients of intensity in flow visualization images indicating mixing level. .... 58

Figure 4.14 Contours of $\omega$ measured $5 \% C$ downstream of trailing edge plane: (a) baseline without actuation, $\tau=$ $3 \% C$; (b) DSJ directly over blade PS $(y=-0.04$ pitch $), C_{\mu, \tau}=0.88, F_{C}{ }^{+}=1.0$. Arrows indicate locations of DSJ holes. 60

Figure 4.15 Exit static pressure with and without NSJ actuation. $\triangleleft$ : DSJ directly over blade PS $(-0.04$ pitch $), C_{\mu, \tau}=$ $0.88, F_{C}^{+}=1.0$ 60

Figure 4.16 Comparison between DSJ (placed near pressure surface of blade) and NSJ (placed near vortex core). $\tau$ $=3 \% C$. Both actuators at $F_{C}{ }^{+}=1.0$. 61

Figure 4.17 Frequency dependence of blockage reduction with DSJ over pressure surface of blade. $\tau=3 \% C \ldots \ldots .62$

Figure 4.18 Endwall blockage vs. pitchwise location of DSJ actuator. $\tau=3 \% C$.

Figure 4.19 Endwall total pressure loss coefficient vs. pitchwise location of DSJ actuator $(\tau=3 \% C$ ): (a) massaveraged $\omega$, (b) stream thrust-averaged (or fully mixed-out) $\omega$.

Figure 4.20 Contribution of each DSJ actuator placed over blade PS. Amplitude is total $C_{\mu, \tau}$ from all working actuators. $\tau=3 \% C$ and ${F_{C}}^{+}=1.0$.

Figure 4.21 Comparison between DSJ and SDJ (Steady Directed Jet). Holes of both actuators placed over pressure surface of blade. $\tau=3 \%$. DSJ at $F_{C}^{+}=1.0$. 64

Figure 4.22 Ratio of endwall loss reduction to expended flow power of directed jet actuation at $\bar{C}_{\mu, \tau}=0.25 \ldots \ldots .65$

Figure 4.23 Estimated recovery rate of expended flow power: (a) as function of relative angle between jet and main flow; (b) as function of velocity ratio for fixed momentum injection. 67 
Figure 5.1 Frequency contents of velocity measured with hot-wire placed in blade exit plane near casing wall. Data taken without actuation.

Figure 5.2 Forced response measured $5 \% C$ downstream of T.E. plane with NSJ over blade tip. $\tau=3 \% C$ and $C_{\mu, \tau}=$ 0.88 .

Figure 5.3 Reduced frequency of periodic unsteadiness of tip clearance vortex. Open and solid symbols denote different flow conditions as summarized in Table 5.1 73

Figure 5.4 Instability of trailing vortices visualized by smoke [5.4]. View from chase airplane 74

Figure 5.5 Schematic of trailing vortex instability 74

Figure 5.6 Dissipation time vs. forcing frequency of trailing vortices in flight test (data from Chevalier [5.4])..... 75

Figure 5.7 Schematic of tip clearance vortex and its image against casing wall creating a pair of counter-rotating vortices similar to trailing vortices. .75

Figure 5.8 Tip clearance vortex core estimated using Rains [5.6] model and location of maximum loss coefficient. Total pressure loss coefficient contour measured near trailing edge plane 77

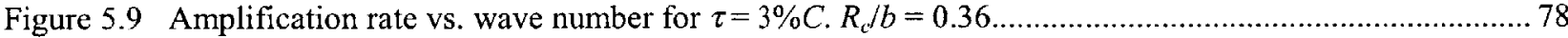

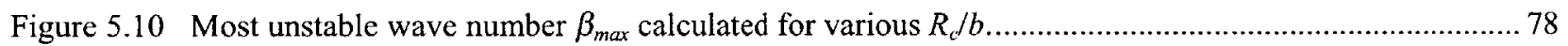

Figure 5.11 Contour plot of most rapidly growing wavelength $\lambda / C$ as function of vortex core radius and separation distance. Symbols are estimations for three tip clearance sizes as labeled. 79

Figure 5.12 Comparison between measured frequency content and estimated frequency based on vortex instability.

Figure 5.13 Vortex core radius $R_{C}$ and separation distance $b$ for two blade chord lengths. Both with $3 \% C$ clearance.

Figure 5.14 Result of thought experiment. Dimensional frequency vs. reduced frequency using blade chord length.

Figure 5.15 Axial view of vortex motion plane. Angle of plane estimated based on trailing vortex instability theory.

Figure 5.16 Variations along chord: (a) variations in $R_{c} / C$ and $b / C$ as function of $\chi / C$; (b) most unstable frequency $F_{C}{ }^{+}$calculated using local approximations. 
Figure 5.17 Time-resolved measurements at various axial locations: (a) locations of hot-wire sensor; (b) power spectral density at each location.

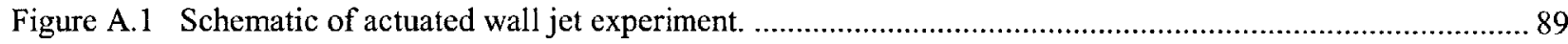

Figure A.2 Hydrodynamic stability calculation for velocity profiles of leakage jet at three downstream locations. 90 Figure A.3 Frequency dependence of mixing enhancement with actuation. Momentum thickness measured at $x=$ $5 \tau$. Actuator slit aligned with pressure surface. Amplitude kept constant at $C_{\mu, \tau}=1.0$. Actuator slit size, $d=$ $1.0 \mathrm{~mm}$

Figure A.4 Amplitude dependence of mixing with actuation. Momentum thickness of shear layer measured at $x=$ $5 \tau$ with actuator slit aligned with suction surface. Non-dimensional forcing frequency, $\beta_{\tau}=0.28$. Tip

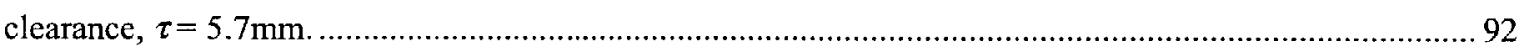




\section{LIST OF TABLES}

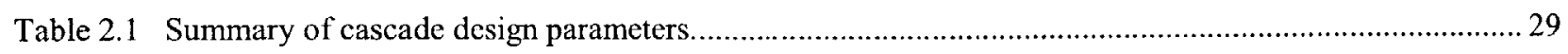

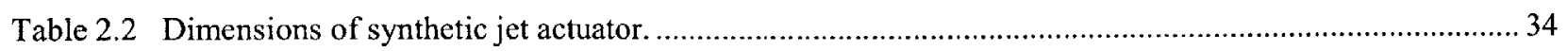

Table 4.1 Effects of each flow process and their combined effects on tip clearance-related blockage and loss explaining observations with NSJ actuator over blade tip.................................................................. 59

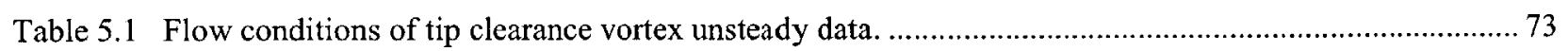




\section{NOMENCLATURE}

\section{SYMBOLS}

\begin{tabular}{|c|c|}
\hline$a$ & Speed of sound or Amplification rate \\
\hline$A_{b}$ & Blocked area due to blockage \\
\hline$A_{b, 2 D}$ & Blocked area related to boundary layers on blade surfaces \\
\hline$A_{b, e w}$ & Endwall blocked area \\
\hline$A_{b, t i p}$ & Tip clearance-related blocked area \\
\hline$A_{e x}$ & Blade passage exit area $=h \times s$ \\
\hline$A_{\text {neck }}$ & Effective neck area \\
\hline$A_{J}$ & Jet area \\
\hline$B$ & Magnitude of magnetic field \\
\hline$b$ & Separation distance between pair of line vortices \\
\hline$C$ & Blade chord \\
\hline$C_{\mu, \tau}$ & Momentum coefficient $=\left(\rho U_{J, \text { peak }}^{2} \delta\right) /\left(\rho U_{L}^{2} \tau\right)$ or $\left(\rho U_{J, p e a k}^{2} A_{J}\right) /\left(\rho U_{\infty}^{2} \tau C\right) \quad$ (in cascade) \\
\hline $\bar{C}_{\mu, \tau}$ & Time-averaged momentum coefficient $=\overline{\left(\rho U_{J}^{2} A_{J}\right)} /\left(\rho U_{\infty}^{2} \tau C\right)$ (in cascade) \\
\hline$C_{D}$ & Discharge coefficient $=Q /(A \sqrt{2 \Delta P / \rho})$ \\
\hline$C_{P}$ & Static pressure coefficient $=\left(P-P_{\infty}\right) / q_{\infty}$ \\
\hline$C_{P, e x}$ & Exit static pressure coefficient measured $5 \% C$ downstream of T.E. plane \\
\hline$d$ & Actuator slit size \\
\hline$f$ & Frequency $(\mathrm{Hz})$ \\
\hline$f_{n}$ & Natural frequency $(\mathrm{Hz})$ \\
\hline$f_{H}$ & Helmholtz frequency $(\mathrm{Hz})$ \\
\hline$F_{C}^{+}$ & Reduced frequency $=f C / U_{\infty}$ (in cascade) \\
\hline$h$ & Span (blade height) \\
\hline
\end{tabular}




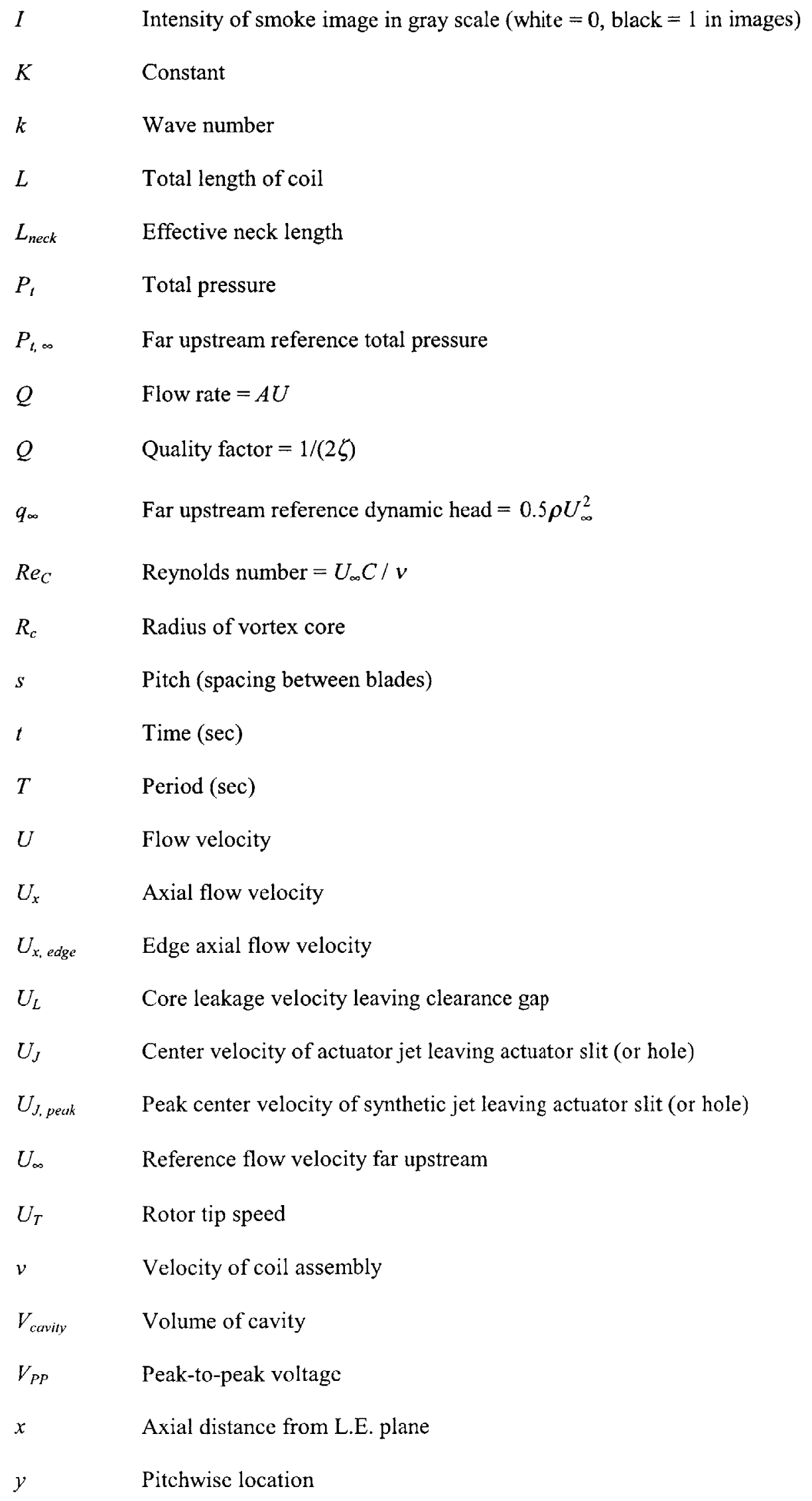




\begin{tabular}{|c|c|}
\hline$\alpha$ & Non-dimensional amplification rate $=a /\left(\Gamma /\left(2 \pi b^{2}\right)\right)$ \\
\hline$\beta_{\text {in }}$ & Cascade inlet flow angle \\
\hline$\beta_{\text {ex }}$ & Cascade exit flow angle \\
\hline$\beta_{\tau}$ & Reduced frequency $=2 \pi f \tau / U_{L}$ or $2 \pi f \tau / U_{\infty}$ (in cascade) \\
\hline$\beta$ & Non-dimensional wave number $=k b$ \\
\hline$\Gamma$ & Circulation \\
\hline$\delta^{*}$ & Displacement thickness \\
\hline$\varepsilon$ & EMF \\
\hline$\zeta$ & Damping ratio \\
\hline$\eta_{J}$ & Ratio of endwall loss reduction to expended flow power \\
\hline$\theta$ & Momentum thickness of shear layer or Inclination angle \\
\hline$\lambda$ & Wavelength \\
\hline$\Pi$ & Flow power \\
\hline$\tau$ & Tip clearance size \\
\hline$\phi$ & Flow coefficient \\
\hline$\chi$ & Chordwise distance from L.E. plane \\
\hline$\psi$ & Pressure coefficient \\
\hline$\omega$ & Total pressure loss coefficient $=\left(P_{t, \infty}-P_{t}\right) / q_{\infty}$ \\
\hline $\bar{\omega}_{e w}^{m}$ & Mass-averaged endwall total pressure loss coefficient \\
\hline $\bar{\omega}_{e w}^{s}$ & Stream thrust-averaged endwall total pressure loss coefficient \\
\hline$\omega_{n}$ & Natural frequency $(\mathrm{rad} / \mathrm{sec})$ \\
\hline$X_{0}$ & Bascline quantity of $X$ (meaning case without actuation) \\
\hline
\end{tabular}




\section{ACRONYMS}

I.D. Inner Diameter

O.D. Outer Diameter

CFD Computational Fluid Dynamics

NSJ Normal Synthetic Jet

DSJ Directed Synthetic Jet

SDJ Steady Directed Jet

L.E. Leading Edge of blade

T.E. Trailing Edge of blade

SLPM Standard Litters Per Minute

FS Full Scale

PS Pressure Surface (or Side)

SS Suction Surface (or Side) 


\section{CHAPTER 1 INTRODUCTION}

\subsection{BACKGROUND}

The relative motion between rotor tips and stationary casing wall in axial compressors requires finite spacing between the two. The most common way to achieve this is to have a small clearance at the rotor tip. The pressure difference across the blade causes a leakage flow through the tip clearance from the pressure surface to the suction surface of the blade as illustrated in Figure 1.1. This tip leakage flow dominates the aero-thermodynamic behavior of the flow in the tip region and has a strong impact on pressure rise capability, compressor efficiency and stability. The influence of tip leakage flow manifests itself in two manners: a blockage that effectively reduces the pressure rise capability and a loss that affects the efficiency of the compressor.

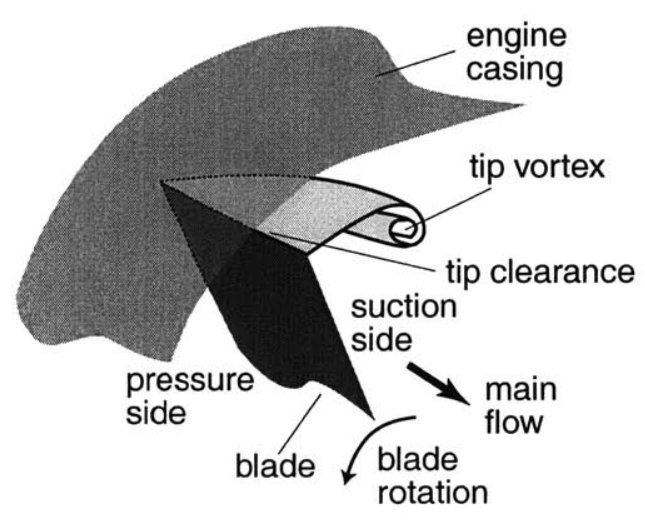

Figure 1.1 Schematic of tip clearance flow.

Measurements from a low-speed multistage (four-stage) compressor with constant casing diameter and annulus height (Wisler [1.1]) illustrate the effects of tip clearance on compressor performance. As shown in Figure 1.2, the tip clearance was increased from the baseline value of $1.38 \%$ to $2.8 \%$ of annulus height, costing $9.7 \%$ in peak pressure rise, $11 \%$ in stalling flow coefficient (flow range), and 1.5 points in peak efficiency.

Smith [1.2] proposed the ratio of the tip clearance to the chord length as a non-dimensional parameter to correlate the effect of tip clearance on the peak pressure rise for axial fans and compressors. As in Figure 1.3, each $1 \%$ increase in clearance/chord results in $4.6 \%$ loss in peak pressure rise. Cumpsty [1.3] describes how in multi- 
stage compressor, not only do the efficiency and pressure ratio deteriorate with increased tip clearance ${ }^{\dagger}$, but the stall line moves so the compressor surges at higher mass flows. All these measured effects are detrimental to the compressor performance.

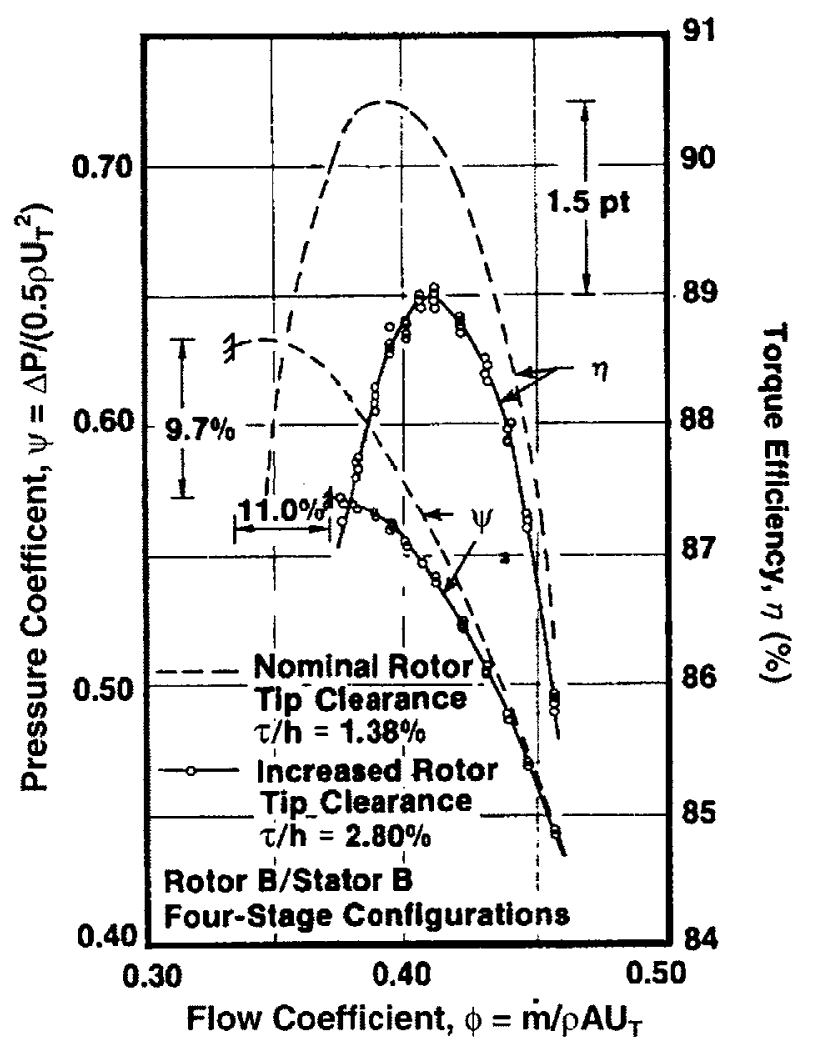

Figure 1.2 Effect of increased tip clearance on overall compressor performance (Wisler [1.1]).

${ }^{\dagger}$ It should be noted that the loss in efficiency and pressure ratio might not be monotonic with increase in tip clearance; for tip clearance below a threshold value, the efficiency and pressure ratio can deteriorate. However, this threshold value is usually smaller than that achieved in practice. 


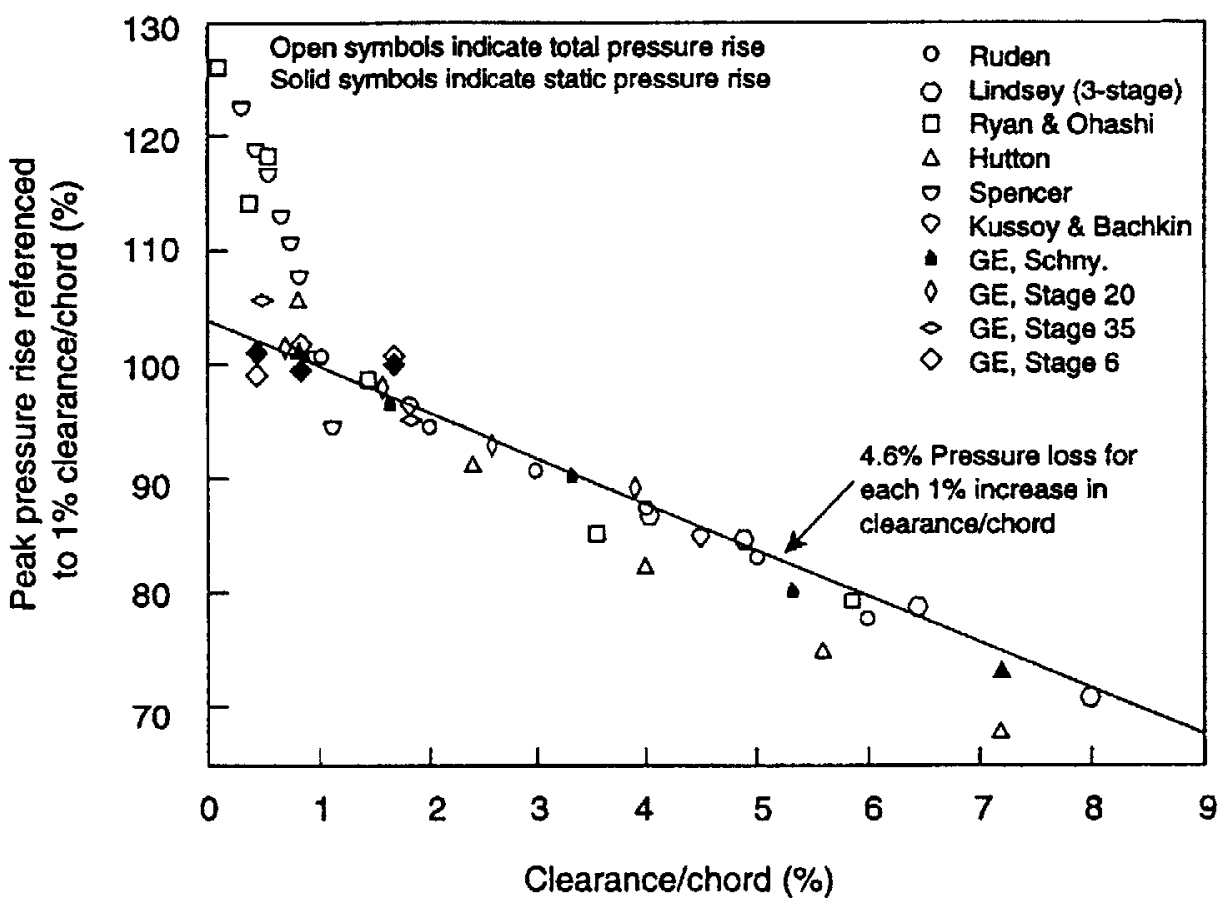

Figure 1.3 Effect of tip clearance on peak pressure rise (Smith [1.2]).

Even though the detrimental effects of large tip clearance are well known, compressors often operate with tip clearances that are larger than aerodynamically desirable due to changes in tip clearance during operations and limitations in manufacturing tolerances. Consequently, there is strong motivation to look for means to relieve the stringent requirement on tight tip clearance and manage the compressor tip clearance flow to minimize its impact on performance. A potential technique for accomplishing this is active flow control and this thesis describes the use of fluidic actuators to beneficially affect the behavior of compressor tip clearance flow.

\subsection{PREVIOUS WORK}

Previous work relevant to this investigation is revicwed in the following. The experimental work of Wisler [1.1], Smith [1.2], Koch [1.4] and others showed the importance of the tip clearance size on the overall compressor performance measure such as the peak pressure rise, stable flow range, and peak efficiency. Other studies such as Inoue et al. [1.5] and Inoue and Kuroumaru [1.6] provided detailed measurements of flow field associated with tip clearance revealing the influence of the clearance size on the endwall flow profiles. 
Much earlier, a pioneering work of Rains [1.7] presented an inviscid model for the leakage flow velocity by relating the tip leakage flow to the blade static pressure difference. He then calculated loss in efficiency associated with tip clearance assuming that the kinetic energy of the leakage flow velocity component normal to the chord cannot be recovered. He also modeled the roll-up process of the tip leakage flow, the motion of the discontinuity surface or the sheet of vorticity as a two-dimensional unsteady flow, as for slender bodies in external aerodynamics.

Storer [1.8] elaborated Rains' model for the tip clearance flow and loss, and constructed a simple model to predict the clearance-related loss using a control volume analysis. In this he viewed the mixing process of the two streams, the leakage jet and the main flow, as the major loss mechanism. He compared the model to the detailed measurements of the tip clearance flow in a low-speed linear cascade complemented by three-dimensional NavierStokes calculations with very satisfactory results.

Chen et al. [1.9] further extended the Rains' view on the tip clearance vortex roll-up process and derived a generalized description of the tip clearance vortex based on a similarity scaling. Good agreement with a wide range of experimental data emphasizes the physical insights that the model provides, i.e. (i) the behavior of the clearance vortex is an inviscid one; (ii) dynamics of the through-flow and the cross-flow associated with the clearance flow field are nearly independent (or decoupled); and (iii) the effect of blade loading is largely determined by the chordwise-averaged loading at the mid-span location and the details of the loading distribution is rather unimportant.

While Rains [1.7], Storer [1.8], and Chen et al. [1.9] focused on understanding of the physical mechanism responsible for the tip clearance-related loss and the roll-up process of the tip clearance vortex, Khalid [1.10] emphasized the importance of the flow blockage in determining the pressure rise capability of axial compressors and developed a method of quantifying it. He constructed a simple model using a description of the growth of a twodimensional turbulent wake in an adverse pressure gradient, which provides insights as to the important processes associated with the growth of the blockage.

Along with the research focused on understanding of the effects and the behavior of the tip clearance flow, there have been efforts secking for a way to alleviate the detrimental effects of the tip clearance flow. Perhaps one of the most successful techniques that mitigates the reductions in the peak pressure rise and the stable flow range associated with large tip clearance is casing treatment; slots or grooves in the casing wall. It has been found however, that its usefulness in improving peak pressure rise and operability is impaired by the reduction in compressor efficiency that it entails. Fujita and Takata [1.11] showed that there is almost a linear relation between 
the stall margin improvement and the rotor maximum efficiency for various types of casing treatments tested. In other words, the general trend is that the more effective a casing treatment configuration is in stall margin improvement, the larger the penalty in compressor efficiency as shown in Figure 1.4. Hitherto there is no data in the open literature containing a treatment configuration that breaks from the curve in Figure 1.4.

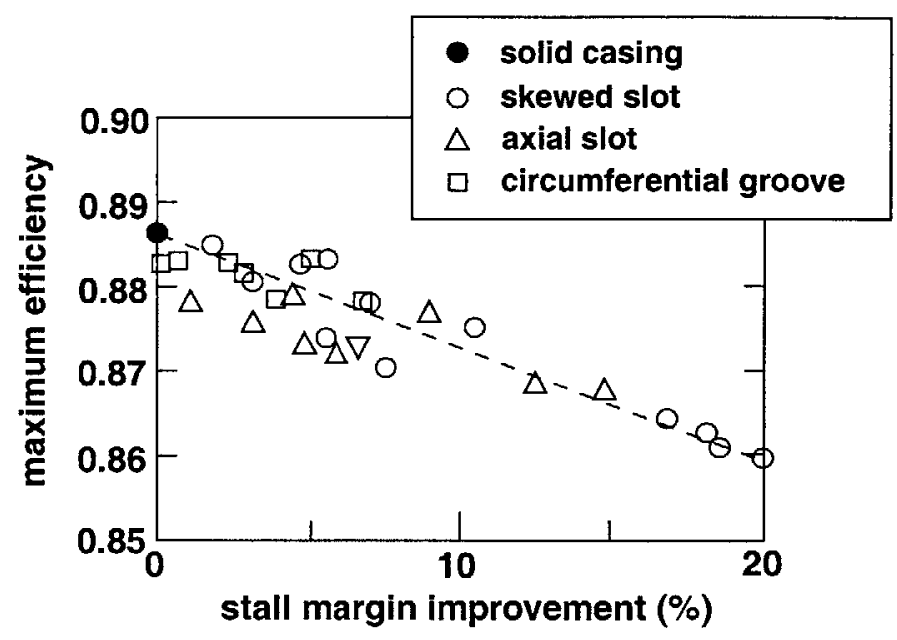

Figure 1.4 Relation between rotor maximum efficiency and stall margin improvement for various casing treatment configurations (Fujita and Takata [1.11]).

A potential technique for accomplishing efficient management of tip clearance flow is active flow control, which has demonstrated its effectiveness in separation control (Seifert et al. [1.12], McCormick [1.13], and Lorber et al. [1.14]). It was found that oscillatory blowing is significantly more effective than steady blowing in controlling separation. For example, Seifert et al. [1.12] observed a total saving of $84 \%$ in momentum coefficient for the same improvement in the airfoil performance as a consequence of the superimposed oscillations. It is conjectured that the entrainment of high momentum fluid to the surface due to large vortical structures, which corresponds to the most unstable mode of the shear layer of separated flow, is responsible for the attachment of the flow. Using a directed synthetic jet actuator (unsteady jet with zero net mass flux, which is directed in the main flow direction), McCormick [1.13] demonstrated an active separation control in a diffuser that achieves about $1600 \%$ return on the input electrical power to the actuator. In other words, the unsteady actuation benefits from great leverage in boundary layer separation control.

The strong impact of the tip clearance on the performance of axial compressors, the limitations of the current techniques in effectively managing the tip clearance flow, and the demonstrated success of active flow control in other applications lead to the technical objectives and approach of this research. 


\subsection{TECHNICAL OBJECTIVES}

The overall goal is to demonstrate the use of fluidic actuators for active tip leakage flow control, which results in improved compressor performance. The specific objectives are:

- Identify aspects of tip clearance flow that can be leveraged through active flow control to benefit compressor performance.

- Design, develop and characterize actuators to provide the desired actuations on tip leakage flow.

- Demonstrate and quantify the effects of developed actuators on the blockage and loss associated with tip leakage flow in a low-speed compressor cascade rig.

- Identify and characterize the change in flow process/processes due to actuations on tip clearance flow.

- Improve understanding of tip clearance flow dynamics using the actuators as diagnostic tools.

\subsection{APPROACH}

The MIT Gas Turbine Laboratory Low-Speed Cascade Rig Facility was used to address the problems. Two sets of experiments have been implemented: the first is baseline experiments for delineating the flow features and performance of the compressor cascade rig with tip clearance changes but no flow control; the second involved use of flow control with different types of flow actuators.

Based on current understanding of how tip clearance flow affects the overall performance of a compressor, the following three measures have been chosen as potential schemes for tip clearance flow control: (i) reduction of the tip leakage flow rate, (ii) mixing enhancement between the defect region of the tip clearance vortcx and the primary stream flow, and (iii) injection of streamwise momentum. Use of three types of fluidic actuators mounted on the casing wall; Normal Synthetic Jet (NSJ; unsteady jet with zero net mass flux normal to the mean flow), Directed Synthetic Jet (DSJ; injection roughly aligned with the mean flow), and Steady Directed Jet (SDJ), is explored to achieve the above mentioned measures. 
The first scheme is to address the problem at the source by reducing the rate of the leakage flow using NSJ as illustrated in Figure 1.5. The objective is to reduce the effective tip clearance by pushing the streamline adjacent to the casing wall using the momentum flux of the NSJ. Because the leakage jet is driven by the pressure difference across the blade, which is largely set by the mid-span loading (Storer [1.8]), reduced clearance size will result in a reduced leakage flow rate.

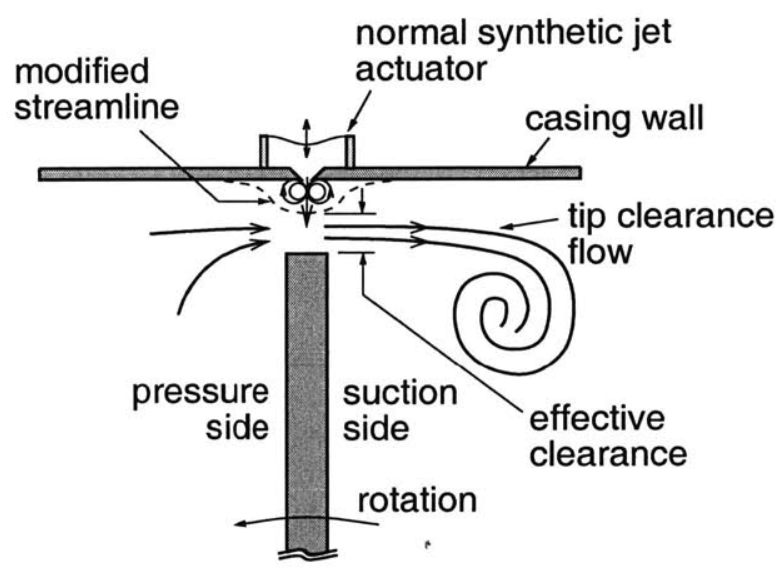

Figure 1.5 Illustration of tip leakage flow rate reduction scheme.

The second scheme, namely mixing enhancement, is to make the wake-like velocity defect region more uniform as illustrated in Figure 1.6, thus reducing the growth of the defect region, which determines the flow blockage in the blade passage. The idea is to exploit a fluid dynamic amplification that may exist to efficiently promote momentum transfer/transport from high momentum main flow to the low momentum flow in the endwall region.

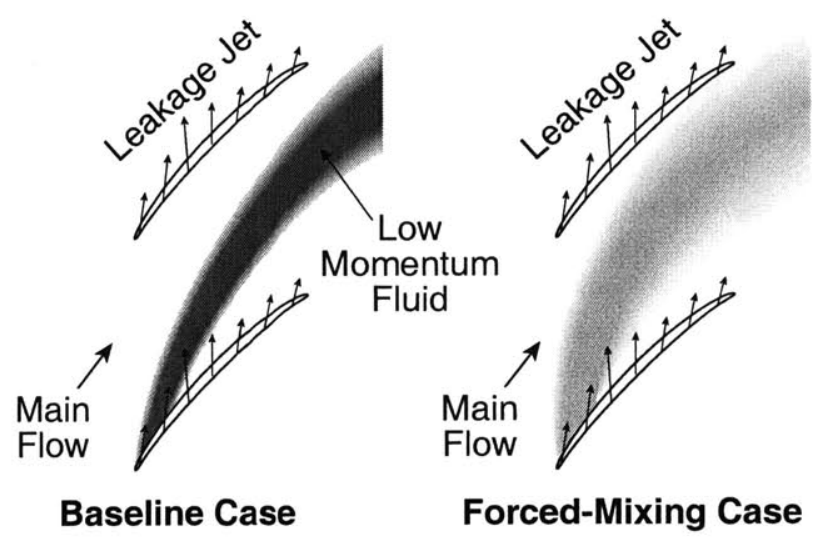

Figure 1.6 Illustration of mixing enhancement scheme. 
The third one (momentum injection) is to energize the retarded flow in the endwall region using momentum flux of the DSJ or SDJ as shown in Figure 1.7. The effects of these three actuation schemes are quantified in the control experiment.

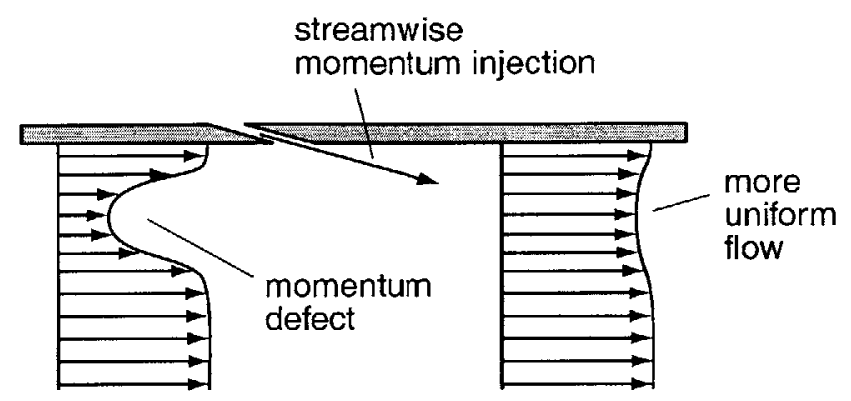

Figure 1.7 Illustration of streamwise momentum injection scheme.

Data consists of flow visualizations using smoke and measurements using pressure as well as hot wire probes. Computations using standard flow solvers and analytical flow modeling are used to help in the design of experiments and interpretation of experimental measurements and observations.

\subsection{CONTRIBUTIONS OF THESIS}

The key contributions of this thesis can be summarized as follows:

- Tip clearance-related blockage reduction using fluidic actuators developed to achieve proposed flow process (or processes) has been experimentally demonstrated in a low-speed compressor cascade rig.

- The effectiveness of each actuator has been quantified and the parametric dependence of the blockage reduction on the actuation amplitude and frequency has been characterized.

- A mechanism that sets the periodic unsteadiness observed in the tip clearance vortex has been proposed and compared with measurements.

- The implications of the experimental results have been described and guidelines for designing future tip clearance flow control schemes have been recommended. 


\subsection{ORGANIZATION OF THESIS}

This thesis is arranged as follows: first Chapter 2 describes the experimental setup, which is followed by a delineation of the baseline performance of the cascade tunnel in Chapter 3. Experimental results to elucidate the time-averaged effects of the actuations and their effectiveness on the behavior of the tip clearance flow are presented next in Chapter 4. Chapter 5 then discusses the time-resolved measurements and the development of a hypothetical flow model to explain the observed periodic flow unsteadiness in the compressor tip leakage flow. Chapter 6 presents overall conclusions and recommendations.

\section{REFERENCES}

[1.1] Wisler, D. C., "Loss Reduction in Axial-Flow Compressors Through Low-Speed Model Testing", ASME J. Eng. Gas Turbines and Power, 1985, vol.107, pp.354-363

[1.2] Smith, L. H., Jr., "The Effect of Tip Clearance on the Peak Pressure Rise of Axial-flow Fans and Compressors", ASME Symposium on Stall, 1958, pp.149-152.

[1.3] Cumpsty, N. A., "Compressor Aerodynamics", Longman Group 1989, pp.345.

[1.4] Koch, C. C., "Stalling Pressure Rise Capability of Axial Flow Compressors", ASME J. Eng. Power, 1981, Vol.103, pp.645-656.

[1.5] Inoue, M., Kuroumaru, M., and Fukuhara, M., "Behavior of Tip Leakage Flow Behind an Axial Compressor Rotor", Journal of Engineering for Gas Turbine and Power, Vol.108, January 1986, pp.7-14.

[1.6] Inoue, M. and Kuroumaru, M., "Structure of Tip Clearance Flow in an Isolated Axial Compressor Rotor", ASME Paper No. 88-GT-251, 1988.

[1.7] Rains, D. A., "Tip Clearance Flows in Axial Flow Compressors and Pumps", California Institute of Technology, Hydrodynamics and Mechanical Engineering Laboratories, Report No. 5. 1954.

[1.8] Storer, J. A., "Tip Clearance Flow in Axial Compressors", Ph.D. dissertation, Department of Engineering, University of Cambridge, January 1991.

[1.9] Chen, G. T., Greitzer, E. M., Tan, C. S., and Marble, F. E., "Similarity Analysis of Compressor Tip Clearance Flow Structure", ASME Paper No. 90-GT-153.

[1.10] Khalid, S. A., "The Effects of Tip Clearance on Axial Compressor Pressure Rise", Ph.D. thesis, MIT, February 1995.

[1.11] Fujita, H. and Takata, H., "A Study on Configuration of Casing Treatment for Axial Flow Compressors", Bulletin of Japanese Society of Mechanical Engineers, Vol.27, No.230, pp.1675-1681, August 1984.

[1.12] Seifert, A., Bachar, T., Koss, D., Shepshelovich, M., and Wygnanski, I., "Oscillatory Blowing: A Tool to Delay Boundary-Layer Separation", AIAA 93-0440, 1993.

[1.13] McCormick, D. C., "Boundary Layer Separation Control with Directed Synthetic Jets", AIAA 2000-0519.

[1.14] Lorber, P. F., McCormick, D. C., Anderson, T. J., Wake, B. E., MacMartin, D. G., Pollack, M. J., Corke, T. C., and Breuer, K. S., "Rotorcraft Retreating Blade Stall Control", AlAA 2000-2475, 2000. 


\section{CHAPTER 2 EXPERIMENTAL SETUP}

\subsection{INTRODUCTION}

To investigate the effect of the proposed actuation schemes on the tip clearance flow in axial compressors, a linear cascade has been designed and fabricated in a low-speed wind tunnel. Because of the cost-effectiveness and experimental simplicity versus rotating rigs, cascade tunnels have been used by many investigators to study tip clearance flows (Storer and Cumpsty [2.1], Heyes et al. [2.2], Bindon [2.3], and Saathoff and Stark [2.4]). One reason for their utility is that the major elements of the problem are the blade loading and the tip clearances. Khalid [2.5] pointed out the dominant role of the tip clearance on endwall flow structure by using a computational method eliminating different physical effects one at a time as shown in Figure 2.1(a) - (d), which show that for this configuration the relative motion of the casing wall or the endwall boundary layer skew results in minor changes in the endwall flow field. In this chapter, we describe the linear cascade facility and its setup for experiments that are designed to assess the utility and effectiveness of actuating the tip clearance flow based on NSJ (Normal Synthetic Jet), DSJ (Directed Synthetic Jet) and SDJ (Steady Directed Jet). 

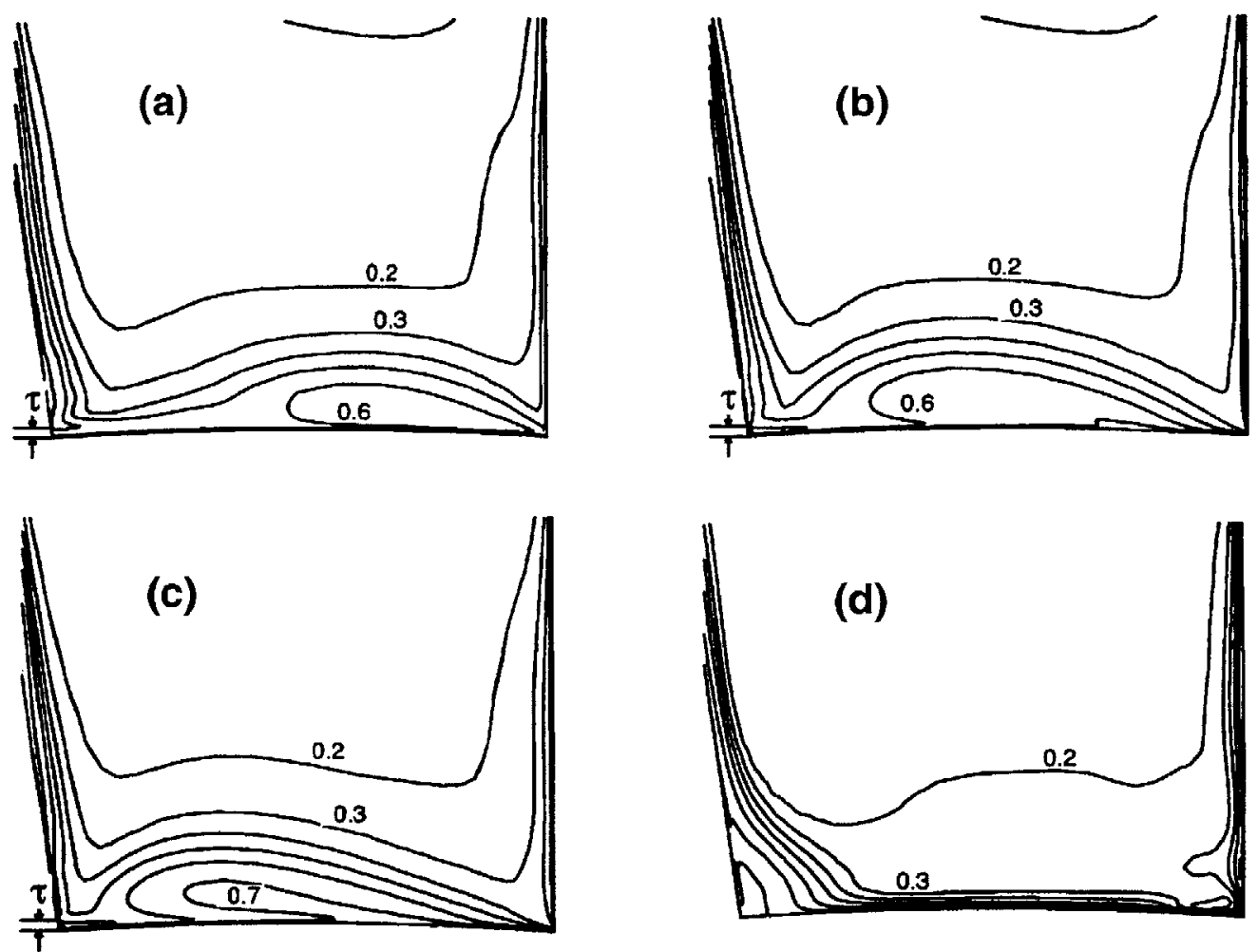

Figure 2.1 Effect of tip clearance on endwall flow structure. Trailing edge total pressure loss coefficient $\omega$ contours of cantilevered stator with (a) moving wall, skewed boundary layer, and clearance; (b) stationary wall, skewed boundary layer, and clearance; (c) stationary wall, collateral boundary layer, and clearance; (d) stationary wall, collateral boundary layer, and no clearance. CFD simulation (Khalid [2.5]).

\subsection{LINEAR CASCADE WIND TUNNEL}

\subsubsection{Test Section}

Table 2.1 summarizes the design parameters of the cascade test section, intended to be geometrically representative of a rotor tip section in a modern aero-engine compressor. The schematic of the cascade test section is shown in Figure 2.2. The inlet of the test section is attached to the $0.30 \mathrm{~m} \times 0.30 \mathrm{~m}$ contraction exit of a wooden wind tunnel settling chamber. The walls of the test section are made out of $9.5 \mathrm{~mm}$ thick Plexiglass $(\mathbb{B}$. There are five blade contour slots on the bottom wall with the blades inserted into the test section from the bottom and held by brackets underneath the bottom wall.

The blades are cast from epoxy in a numerically machined aluminum mold so that hypodermic tubes (with O.D. $0.90 \mathrm{~mm}$ and I.D. $0.66 \mathrm{~mm}$ ) can be embedded for blade surface static pressure measurements. Two blades are 
instrumented with 14 static pressure taps each, one at mid-span and the other near tip (1.5\%C from the blade tip). All the blades are interchangeable and three central blades in the test section are cantilevered at the roots to allow variable tip clearances. A $7 \mathrm{~mm}$ wide transition strip made out of 50-grit sandpaper is glued on the suction surface of each blade at $42 \% \mathrm{C}$ from the leading edge to allow operating at low Reynolds number without laminar separation (see section 3.1). The outlet screen pressurizes the test section so that wall boundary layers can be removed by bleeding to atmospheric pressure through slots. There are endwall bleed slots on the top and bottom walls near the inlet of the test section as shown in the schematic. Periodicity is achieved by adjusting the bleed ports on the sidewalls.

The jet actuator is attached to the endwall as shown in Figure 2.2 and sits in a slot machined through the casing wall. Detailed description of the jet actuator is in section 2.3. The angle of the two exit sidewalls downstream of the blades is set by the mid-span exit flow angle as obtained from the MISES code [2.6].

Table 2.1 Summary of cascade design parameters.

\begin{tabular}{|l|l|}
\hline Airfoil & G.E. $\mathrm{E}^{3}$ rotor B tip section \\
\hline Chord, $C$ & $0.190 \mathrm{~m}$ \\
\hline Pitch, $s$ & $0.177 \mathrm{~m}$ \\
\hline Span, $h$ & $0.305 \mathrm{~m}$ \\
\hline Camber & $30.7^{\circ}$ \\
\hline Stagger & $56.9^{\circ}$ \\
\hline Reynolds number & $\geq 1.0 \times 10^{5}$ \\
\hline Inlet flow angle, $\beta_{i n}$ & $62.7^{\circ}$ \\
\hline Exit flow angle, $\beta_{e x}$ & $51.8^{\circ}$ \\
\hline Diffusion factor & 0.40 \\
\hline
\end{tabular}




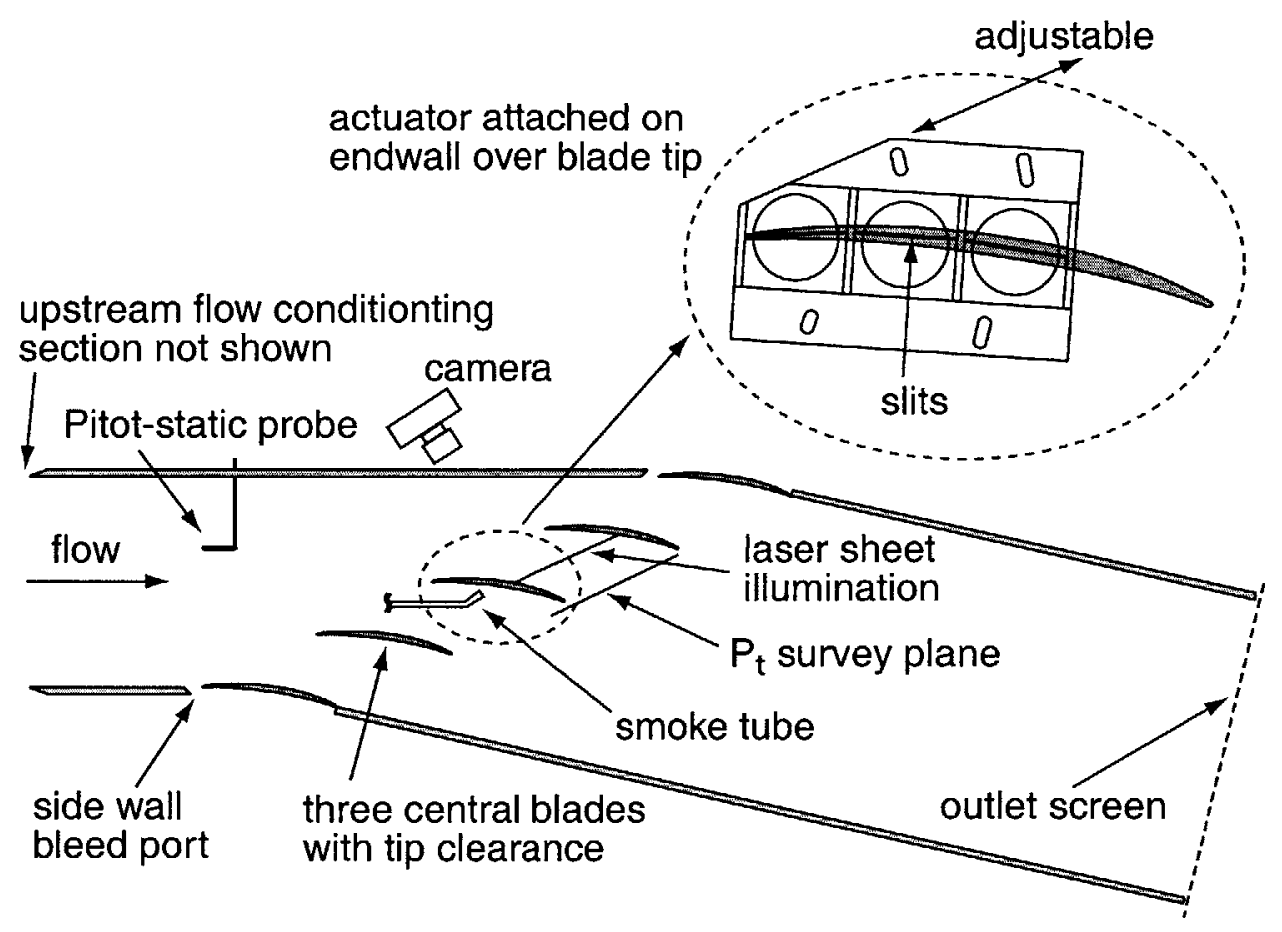

Figure 2.2 Schematic of cascade wind tunnel test section.

\subsubsection{Instrumentation}

All the data are taken using an Intel( Pentium $^{\circledR}$ II $266 \mathrm{MHz}$ PC running on Microsoft( Windows $\mathrm{NT}\left({ }^{\circledR}\right.$ 4.0. The PC is equipped with a data acquisition card (PCI-6071E) manufactured by National Instruments ${ }^{\mathrm{TM}}$. The data acquisition board has $1.25 \mathrm{MS} / \mathrm{sec}$ maximum sampling rate, 12-bit resolution, and 32 differential channels for $\mathrm{A} / \mathrm{D}$ conversion. Uncertainties in each measurement are estimated by small-sample method (Kline and McClintock [2.7]) with odds 20:1 and are presented as error bars in each data plot throughout the document.

The blade surface static pressure distribution is measured using blades instrumented with the static pressure taps described earlier. A $3.2 \mathrm{~mm}$ outer diameter Pitot-static probe manufactured by United Sensor located about $2.1 \mathrm{C}$ upstream of the blade leading edge plane is used to measurc far upstream reference conditions. The static pressure rise across the blade passage is measured by averaging six equally spaced static pressure taps drilled onto the casing endwall along the line $5 \% \mathrm{C}$ downstream of the trailing edge plane of the third blade passage from the bottom in Figure 2.2.

To assess the effects of actuation on the time-averaged performance of the compressor cascade, total pressure is surveyed using a Kiel probe in the survey plane, $5 \% \mathrm{C}$ downstream of the trailing edge plane as shown in Figure 2.2. The Kiel probe with $3.2 \mathrm{~mm}$ outer diameter head manufactured by United Sensor is attached to a three-axis 
TSI® traverse table model 9400 , which has $0.01 \mathrm{~mm}$ position resolution with built-in backlash compensation loop. The Kiel probe measures the total pressure within $1 \%$ of the actual value up to $\pm 48^{\circ}$ yaw angle and $\pm 45^{\circ}$ pitch angle of the flow allowing total pressure measurements without rotating or aligning the probe with the local flow direction. The traversing is programmed and performed automatically over the survey area $(1$ pitch $\times 0.5 \mathrm{span})$ with a mesh size of 19 (pitchwise) $\times 13$ (spanwise).

Two Setra pressure transducers Model 239 with $0-2.5^{\prime \prime} \mathrm{WC}$ (or $623 \mathrm{~Pa}$ ) are used to measure differential pressures. One is dedicated to measure the far upstream reference dynamic head from the Pitot-static probe. The other is used to measure either the total pressure loss coefficient, $\omega$ or the static pressure coefficient, $C_{P}$. The Setra pressure transducers have $\pm 0.14 \% \mathrm{FS}$ accuracy, which is $\pm 2.2 \% q_{\infty}$ at $R e_{C}=100,000$.

Along with time-averaged measurements, unsteady measurements are also taken to examine the mechanism of the actuation. A DANTEC single $5 \mu \mathrm{m}$ thick hot-wire (probe type $55 \mathrm{P} 11$ ) with DANTEC CTA bridge $56 \mathrm{C} 17$ is used to take unsteady measurements. The hot-wire is held by a holder made out of a $1.27 \mathrm{~cm}$ diameter $\mathrm{Al}$ tube, which is attached to the traverse table that positions the hot-wire. The holder extends about $17 \mathrm{~cm}$ upstream to minimize the interference. The signal from the hot-wire anemometer is filtered through an anti-aliasing low-pass filter (Frequency Devices $901 \mathrm{~F}$ ) before sampled by the data acquisition card. The hot-wire anemometer is also used in characterizing the jet actuators as described in subsection 2.3.2.

\subsubsection{Flow Visualization}

Flow visualization has been used to interrogate the changes in the behavior of the tip clearance flow with actuation. The primary interest is the change in mixing level of the leakage jet with main flow due to actuation.

The setup for the flow visualization is shown in Figure 2.2. The interrogation plane is $55 \% \mathrm{C}$ from the leading edge and is parallel to the leading edge plane. A cylindrical lens is used to pan a beam from a He-Ne laser illuminating the plane through the bottom wall of the test section. The laser rated Class III-b is a product of MellesGroit with $15 \mathrm{~mW}$ maximum output at $632.8 \mathrm{~nm}$. The actual output is about $7 \mathrm{~mW}$. Flow is seeded with smoke generated by burning incense sticks in a small separate chamber fed by regulated and metered shop air. The outlet of the smoke chamber is connected to a metal tube (with O.D. of $6.4 \mathrm{~mm}$, labeled as "smoke tube" in Figure 2.2), which releases the smoke in the test section. The flattened exit of the tube is placed near the entrance of the tip clearance on the pressure side and is directed parallel to the laser sheet as shown in Figure 2.2 as the local leakage jet is 
approximately in the same direction. The flow rate of the shop air into the smoke chamber is set such that the velocity at the exit of the tube is the same as the far upstream velocity so as to be more or less iso-kinetic with the wind tunnel flow. By placing the tube on the pressure side of the tip gap, the leakage jet is marked with the smoke. Acceleration near the entrance of the clearance attenuates the disturbance caused by the smoke tube.

A Nikon FM2 35mm camera is placed outside the test section as shown in Figure 2.2. Kodak TMAX P3200 films are used at the shutter speed of $2(0.5 \mathrm{sec}$ exposure) with f-stop of 2.8 , i.e. fully opened aperture. Processed images only provide time-averaged flow structures due to the long exposure time.

\subsection{ACTUATOR}

\subsubsection{Actuator Design and Fabrication}

Since actuator amplitude and the bandwidth requirements for tip clearance flow control are not known in advance, the desire for the jet actuator is to maximize both the amplitude and bandwidth. To maximize amplitude and bandwidth, two design guidelines are set: the first is to allow maximum stroke of the voice coil for a given area ratio and the second is to minimize the cavity volume for given geometrical restrictions to push the Helmholtz resonance frequency (Dowling and Ffowcs Williams [2.8]) as high as possible.

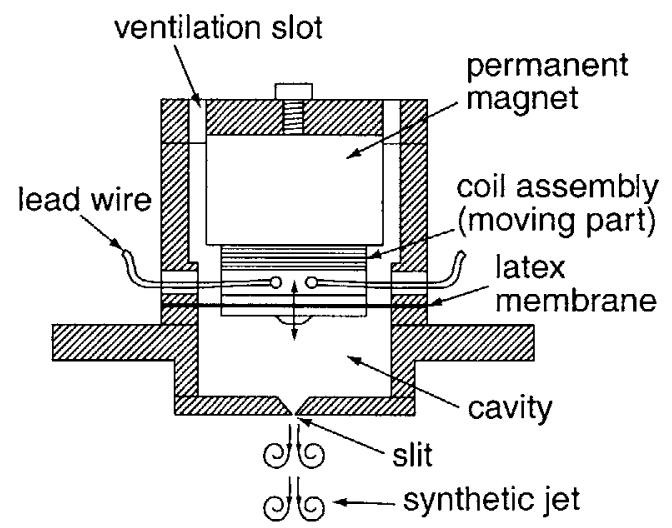

Figure 2.3 Schematic of synthetic jet actuator used in cascade rig. Configuration of Normal Synthetic Jet actuator with slit.

The schematic of the actuator used is shown in Figure 2.3. This actuator is widely known as synthetic jet actuator (Amitay et al. [2.9], Smith et al. [2.10]) and it consists of a vibrating membrane, a cavity, and a slit (in case of Normal Synthetic Jet or NSJ) or holes (in case of Directed Synthetic Jet or DSJ). There are three isolated 
synthetic jet actuators inline in the direction normal to Figure 2.3. The backside of the membrane is open to the atmosphere because the static pressure in the wind tunnel test section is small enough (the difference is about $1 \%$ of atmospheric pressure) so back pressurization is not necessary (See Figure 2.2 and Figure 2.5 for a top view of the installed actuator and Figure 2.4 for a side view).

The Normal Synthetic Jet actuator has three straight slits that are shaped to approximately follow the camberline of the blade (Figure 2.2) while the Directed Synthetic Jet actuator has four tilted holes per actuator (12 holes total) that are directed approximately to the chordwise direction and tilted by $25^{\circ}$ from the casing wall (Figure 2.4 and Figure 2.5). The actuator covers the first $70 \% C$ from the leading edge because most of the benefit associated with actuation is expected to be attained near the leading edge (Khalid et al. [2.11]). To study the dependence on pitchwise location of the actuator, the casing wall with the actuator has been made adjustable in the pitchwise direction as indicated in Figure 2.2 and Figure 2.5. The dimensions of the actuator are summarized in Table 2.2.

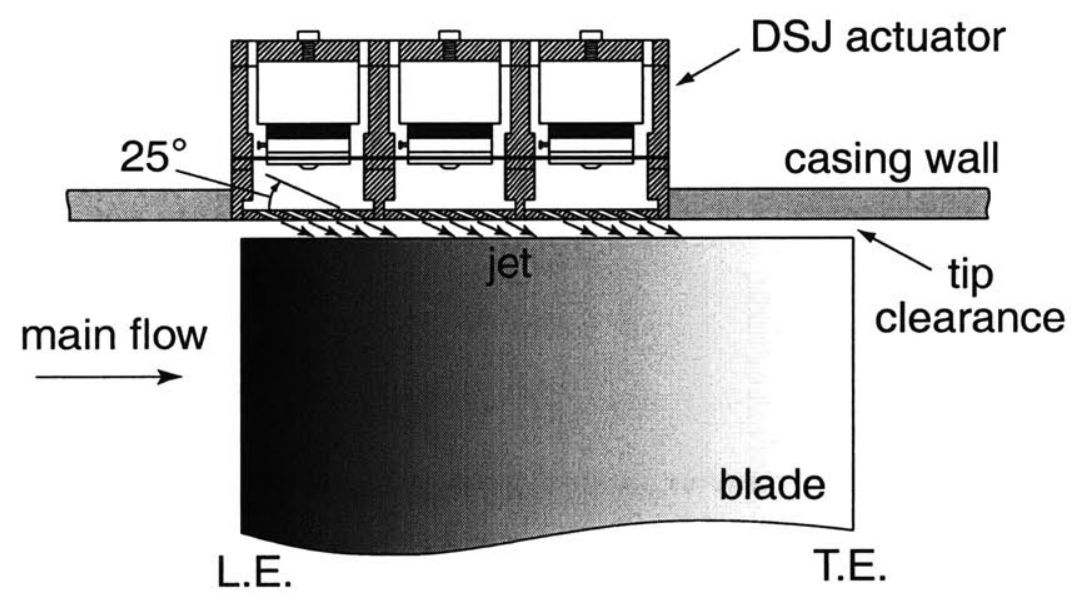

Figure 2.4 Schematic of DSJ actuator mounted on casing wall. 

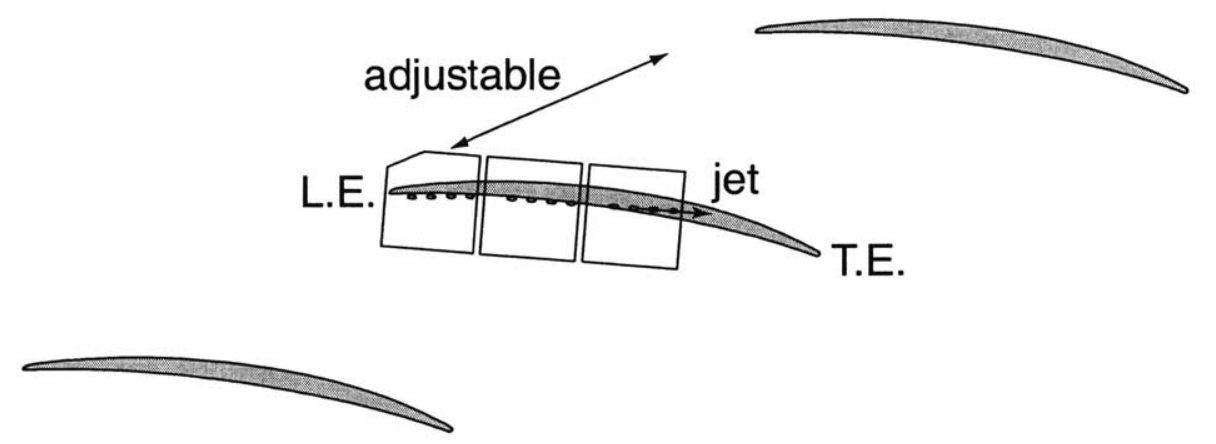

Figure 2.5 Schematic of Directed Synthetic Jet (DSJ) actuator mounted on casing wall showing the direction of the jet.

Because of compact geometry requirements, BEI Kimco voice coil actuators (LA13-12-000A) are used to drive the membrane. The voice coil actuator has $15.5 \mathrm{~N}$ peak force, $\pm 3.18 \mathrm{~mm}$ stroke, and 14 gram coil assembly. To allow for maximum excursion of the voice coil, a $0.51 \mathrm{~mm}$ thick latex sheet is used for the membrane. The latex membrane provides sealing of the cavity and positioning of the coil assembly in the center relative to the permanent magnet. The membrane is pre-stretched around a wooden frame and holes on the membrane for fastening screws are die-cut. It is then sandwiched and fastened to the $\mathrm{Al}$ housing shown in Figure 2.3 with hatches and also to the coil assembly between two disks that are $2.6 \mathrm{~cm}$ in diameter. The membrane is finally freed from the wooden frame. The pre-stretch is necessary to avoid wrinkles on the membrane while tightening screws. A sinusoidal signal generated with a WAVETEK function generator model 29 is amplified with Yorkville AP4040 audio amplifiers and then fed to the voice coil actuator.

Table 2.2 Dimensions of synthetic jet actuator.

\begin{tabular}{|l|l|}
\hline External length & $136.5 \mathrm{~mm}$ \\
\hline External width & $47.6 \mathrm{~mm} / 85.7 \mathrm{~mm}$ (including mounting flanges) \\
\hline External height & $56.1 \mathrm{~mm}$ \\
\hline Cavity volume per actuator & $1.52 \times 10^{4} \mathrm{~mm}^{3}$ \\
\hline Slit/Hole plate thickness & $3.18 \mathrm{~mm}$ \\
\hline Slit width (NSJ) & $0.254 \mathrm{~mm}$ \\
\hline Slit area per actuator (NSJ) & $10.5 \mathrm{~mm}^{2}$ \\
\hline Hole diameter (DSJ) & $1.59 \mathrm{~mm}$ \\
\hline Hole length (DSJ) & $7.51 \mathrm{~mm}$ \\
\hline Hole area per actuator (DSJ) & $7.92 \mathrm{~mm}^{2}$ \\
\hline
\end{tabular}


For Steady Directed Jet (SDJ) actuation, the housing of the voice coil actuator and membrane is replaced with a plenum that supplies regulated shop air to the 12 holes on the plates. The flow rate into the plenum is regulated using a pressure regulator and a needle valve and is measured using a Teledyne Electronic Technologies HFM-201 flow meter with a range of $0-300$ SLPM of air and $0-5 \mathrm{~V}$ output.

\subsubsection{Actuator Performance Characterization}

The amplitude/frequency response of the actuator (NSJ and DSJ) is characterized by measuring the jet velocity at the exit of the slit or the hole with a DANTEC hot-wire anemometer. The measurements are carried out on a separate test bench. The hot-wire is positioned using a manual $\mathrm{X}-\mathrm{Y}-\mathrm{Z}$ axis precision positioning stage isolated from the test bench that holds the actuator. The measured response of the NSJ actuator is shown in Figure 2.6. The peakto-peak driving voltages of the voice coil are labeled in the figure. The peak center jet velocity, $U_{J, \text { peak }}$ on the vertical axis is determined from an ensemble-average of 20 jet velocity cycles measured at the center of the jet. The error bar in Figure 2.6(a) represents an estimated 95\% confidence interval of the velocity measurement. Using a linear interpolation of the measurements, an input voltage for the cascade actuation experiment is calculated for a desired jet velocity $U_{J, \text { peak }}$ at a desired frequency $f$ within the envelope defined by the maximum input voltage to the voice coil. Since the total pressure of the jet created by the actuator is 20 to 30 times the static pressure variation in the cascade test section, it is assumed that the actuator performance remains unchanged during the wind tunnel testing. Post-calibration of the actuator taken after a usage about 50 hours on the cascade experiment showed no significant changes in the actuator performance as the difference in the velocity measurements was only on the order of the measurement uncertainty as shown in Figure 2.6(a). However, the membrane has to be replaced from time to time, because it breaks along the sandwiched edges after being used for more than about 50 hours. There are membrane-to-membrane variations in the response because the amount of pre-stretch has not been controlled precisely and a new amplitude/frequency response is measured every time the membrane is replaced. 

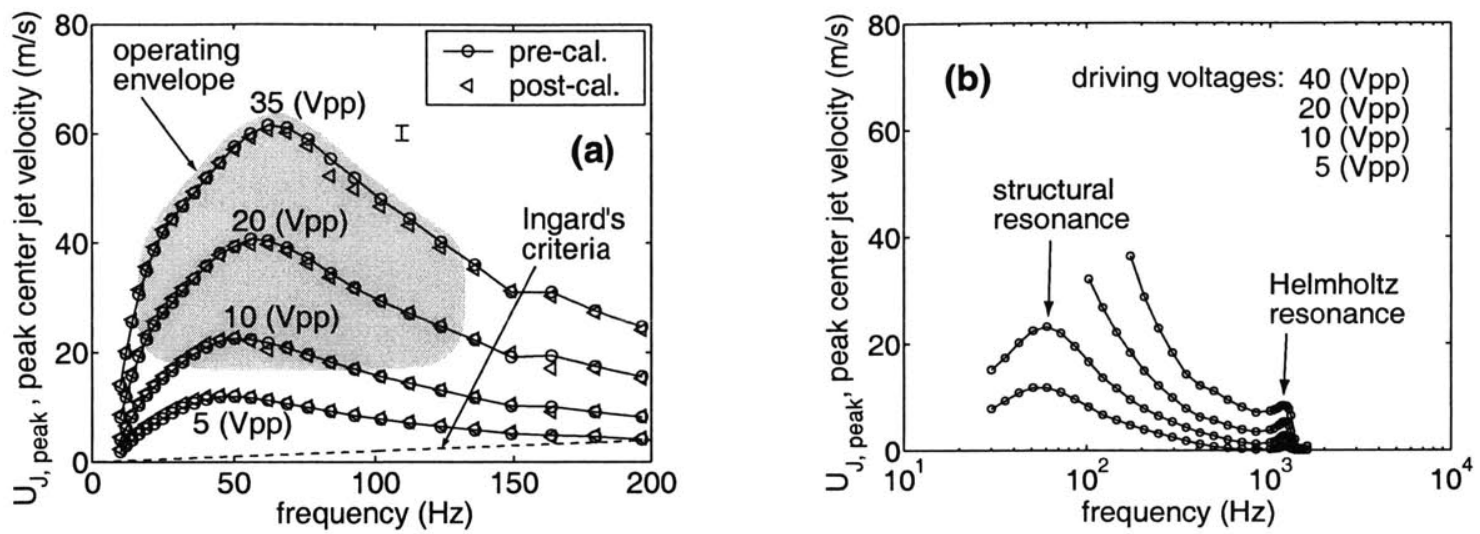

Figure 2.6 Amplitude/frequency response of NSJ actuator: (a) comparing pre- and post-calibration on linear scale abscissa; (b) calibration taken over larger frequency range shown on log scale abscissa. Actuator slit size $d=0.25 \mathrm{~mm}$. Vpp is peak-to-peak driving voltage. Data of two different membranes.

The structural resonance frequency of the voice coil and membrane system was measured using an impulse test without cavity and slit/hole plates. The coil assembly is tapped with a hammer and the back EMF ( $\varepsilon=B L v$ where $B$ is the magnitude of the magnetic field, $L$ is the total length of coil, and $v$ is the velocity of the coil assembly) generated by the movement of the voice coil is recorded. The measured time trace is compared to a simulated time trace based on the standard second-order model:

$$
H(s)=\frac{K}{s^{2}+\frac{\omega_{n}}{Q} s+\omega_{n}^{2}}
$$

The simulated time trace with natural frequency, $f_{n}=\omega_{n} /(2 \pi)=54.7 \mathrm{~Hz}^{\ddagger}$ and the quality factor, $Q=1 /(2 \zeta)=4.4$ matches the measurement well as shown in Figure 2.7. The peak near $55 \mathrm{~Hz}$ in the actuator calibration shown in Figure 2.6(a) and (b) thus corresponds to the structural resonance. The frequency of the peak increases by up to about $20 \mathrm{~Hz}$ as the driving voltage increases. The built-in high-pass filter in the audio amplifier, with cutoff frequency of $20 \mathrm{~Hz}$, results in roll-off of the actuator jet velocity below the cutoff frequency as shown in Figure 2.6(a).

\footnotetext{
$\ddagger$ As mentioned above, the structural resonance frequency $f_{n}$ varies slightly depending on the amount of pre-stretch applied to the membrane.
} 


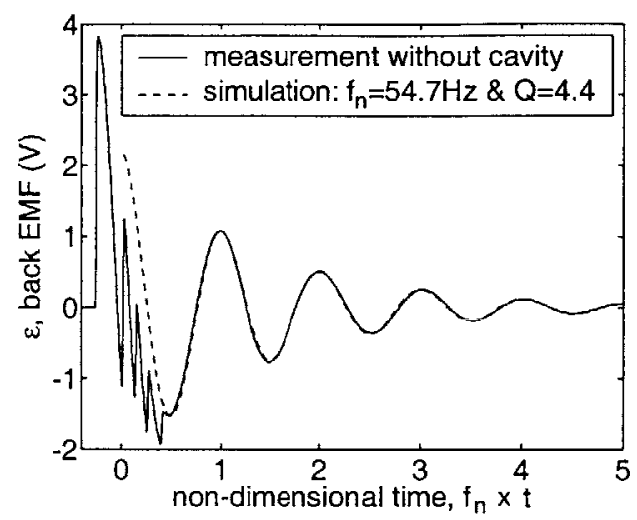

Figure 2.7 Comparison between measured and simulated impulse responses of voice coil and membrane system.

The Helmholtz resonance frequency, $f_{H}[2.8]$ is

$$
f_{H}=\frac{a}{2 \pi} \sqrt{\frac{A_{n e c k}}{L_{n e c k} \cdot V_{\text {cavity }}}}
$$

where $a$ is the speed of the sound, $A_{\text {neck }}$ is the area of the neck (slit or holes), $L_{\text {neck }}$ is the length of the neck, and $V_{\text {cavity }}$ is the volume of the cavity (See Figure 2.3). Assuming the thickness of the slit plate is the effective length of the neck, the predicted Helmholtz frequency, $f_{H}$ is $800 \mathrm{~Hz}$ based on Table 2.2. However, the chamfered slit would reduce the effective length of the neck and consequently increase the Helmholtz frequency. Therefore, the second peak in the measured actuator response at around $1200 \mathrm{~Hz}$ as shown in Figure $2.6(\mathrm{~b})$ is due to the Helmholtz resonance. Above the Helmholtz resonance peak, the actuator response rolls off quickly to zero. It turns out that the Helmholtz resonance frequency is high enough to allow bandwidth necessary for the cascade experiments. The gray shaded area in Figure 2.6(a) is the operating envelope of the actuator used in the cascade experiments.

Ingard [2.12] identified different operating regimes of synthetic jets. At sufficiently high levels of excitation, the amplitude of the particle displacement in the neck exceeds the length of the neck. The dividing line,

$$
U_{J, \text { peak }}=2 \pi f L_{\text {neck }}
$$

is plotted in Figure 2.6(a) as a dashed line. The actuator operates well above the line meaning that it is in the acoustic streaming regime that generates discrete vortices.

Figure 2.8 shows the amplitude/frequency response of DSJ actuator. It is similar to that of NSJ shown above with a peak near the structural resonance frequency. Ensemble-averaged time-traces of DSJ velocity and momentum over a period are shown in Figure 2.9. A directed jet is formed during an injection stroke, while ingestion from all directions is created during a suction stroke (the peak during the suction stoke is smaller than that during the 
injection stroke because of the small distance - less than half of the hole diameter - between the exit of the hole and the hot-wire sensor). Only the injection stroke contributes to the directed momentum and the time-averaged momentum,

$$
\overline{\left(\rho U_{J}^{2} A_{J}\right)}=0.28 \times\left(\rho U_{J, p e a k}^{2} A_{J}\right)
$$

is indicated in Figure 2.9(b). The time-averaged momentum is used to compare DSJ with Steady Directed Jet (SDJ).

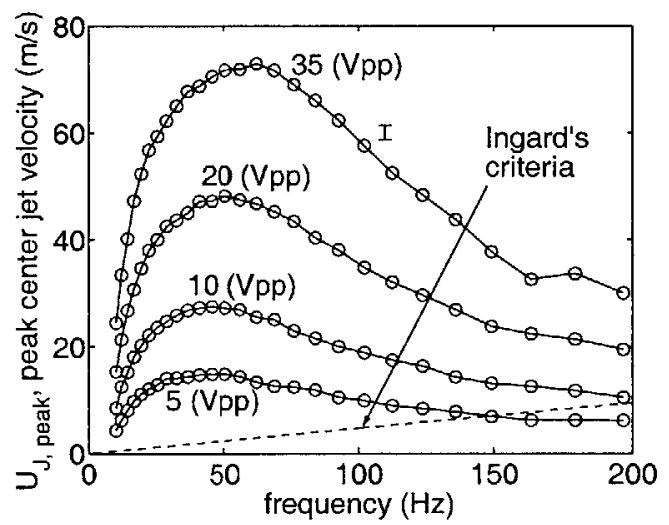

Figure 2.8 Amplitude/frequency response of DSJ actuator.
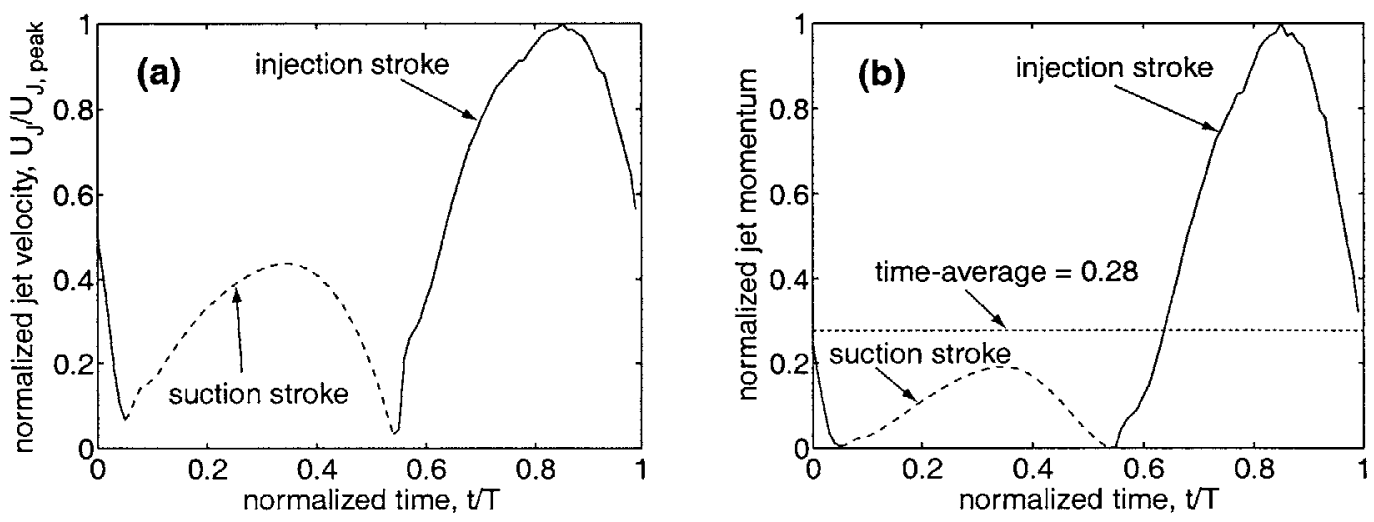

Figure 2.9 Time-trace of DSJ: (a) velocity; (b) momentum. Ensemble-average of 20 cycles.

The calibration of the Steady Directed Jet (SDJ) actuator is shown in Figure 2.10. The jet velocity is again measured with a hot-wire placed at the exit of the actuator hole. In the cascade experiment, the desired jet velocity is achieved by setting the flow rate (or the voltage readout of the Teledyne flow meter) based on the least square fit calibration line in Figure 2.10. 


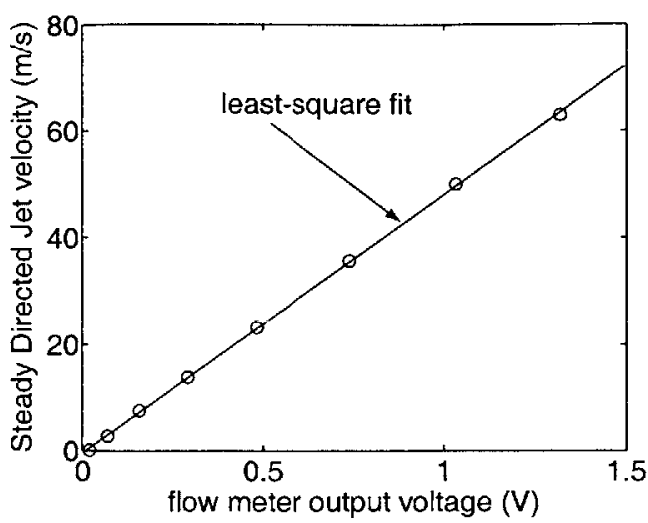

Figure 2.10 Calibration of Steady Directed Jet (SDJ) actuator.

\section{REFERENCES}

[2.1] Storer, J. A. and Cumpsty, N. A., "Tip Leakage Flow in Axial Compressors", ASME Paper No. 90-GT-127, 1990.

[2.2] Heyes, F. J. G., Hodson, H. P., and Dailey, G. M., "The Effect of Blade Tip Geometry on the Tip Leakage Flow in Axial Turbine Cascades", J. of Turbomachinery, July 1992, pp.643-651.

[2.3] Bindon, J. P., "The Measurement and Formation of Tip Clearance Loss", J. of Turbomachinery, July 1989, pp.257-263.

[2.4] Saathoff, H. and Stark, U., "Endwall Boundary Layer Separation in a High-Stagger Compressor Cascade and a Single-stage Axial-Flow Low-Speed Compressor", Forschung im Ingenieurwesen, Vol. 65 Issue 8, 2000, pp. 217-216, Springer.

[2.5] Khalid, S. A., "The Effect of Tip Clearance on Axial Compressor Pressure Rise", Gas Turbine Laboratory, Massachusetts Institute of Technology, Ph.D. Thesis, February 1995.

[2.6] Drela, M. and Youngren, H., "A User's Guide to MISES 2.1", MIT Computational Aerospace Science Laboratory, 1995.

[2.7] Kline, S. J. and McClintock, F. A., "Describing Uncertainties in Single-Sample Experiments", Mechanical Engineering, January 1953, pp.3-8.

[2.8] Dowling, A. P. and Ffowcs Williams, J. E., "Sound and Sources of Sound", Ellis Horwood Limited, 1983.

[2.9] Amitay, M., Honohan, A., Trautmann, M., and Glezer, A., "Modification of the Aerodynamic Characteristics of Bluff Bodies Using Fluidic Actuators", AIAA 97-2004, 1997.

[2.10] Smith, D. R., Amitay, M., Kibens, V., Parekh, D., and Glezer, A., "Modification of Lifting Body Acrodynamics Using Synthetic Jet Actuators", AIAA 98-0209, 1998.

[2.11] Khalid, S. A., Khalsa, A. S., Waitz, I. A., Tan, C. S., Greitzer, E. M., Cumpsty, N. A., Adamczyk, J. J., and Marble, F. E., "Endwall Blockage in Axial Compressors", ASME Paper No. 98-GT-188.

[2.12] Ingard, U., "On the Theory and Design of Acoustic Resonator", J. of the Acoustical Society of America, Vol. 25, No. 6, November 1953. 


\section{CHAPTER 3 BASELINE PERFORMANCE}

The measurements presented in this chapter serve as the baseline results to which the experiments with flow control (discussed in the next chapter) can be compared for assessing the utility of proposed flow control strategies.

\subsection{EFFECT OF REYNOLDS NUMBER}

It is desirable to operate the cascade wind tunnel at a speed as low as feasible so that the amplitude and bandwidth requirement of the actuator is minimized. However, the Reynolds number of the cascade needs to be high enough to ensure well-behaved boundary layers without extensive laminar separation. This is more representative of the flow in an actual compressor. Surveys of the total pressure loss coefficient have been carried out at various Reynolds numbers by varying upstream flow velocity. The contours of the total pressure loss coefficients,

$$
\omega=\frac{P_{t, \infty}-P_{t}}{q_{\infty}}
$$

measured with the Kiel probe $5 \% \mathrm{C}$ downstream of the trailing edge plane are shown in Figure 3.1(a) - (e). The tip clearance is $3 \% \mathrm{C}$ and the survey area is 1 pitch $\times 0.5$ span. The interval between contour lines is $10 \%$ of far upstream reference dynamic head $\left(0.1 q_{\infty}\right)$. The uncertainty in $\omega$ is \pm 0.034 with $95 \%$ confidence. At $R e_{C}=50,000$ (Figure 3.1(a)), the flow on the blade surface is not fully attached due to a laminar separation causing a thick blade wake with large total pressure loss and blockage. The design blade loading (or the pressure difference across the blade) is not established resulting in a tip clearance vortex smaller and closer to the blade suction surface than those at higher $R e_{C}$. As the Reynolds number increases, the boundary layer on the blade surface becomes thinner. The total pressure loss coefficient contours become insensitive to the Reynolds number above 100,000.

The integrated flow blockage is computed ${ }^{\S}$ and plotted against the Reynolds number as shown in Figure 3.2 . The blockage increases rapidly when $R e_{C}$ is reduced below 100,000 , while it is nearly constant when $R e_{C}>100,000$. Therefore, all the quantitative measurements presented in the following have been done above the Reynolds number of 100,000 .

\footnotetext{
${ }^{\S}$ Section 3.4 describes how the blockage is computed.
} 

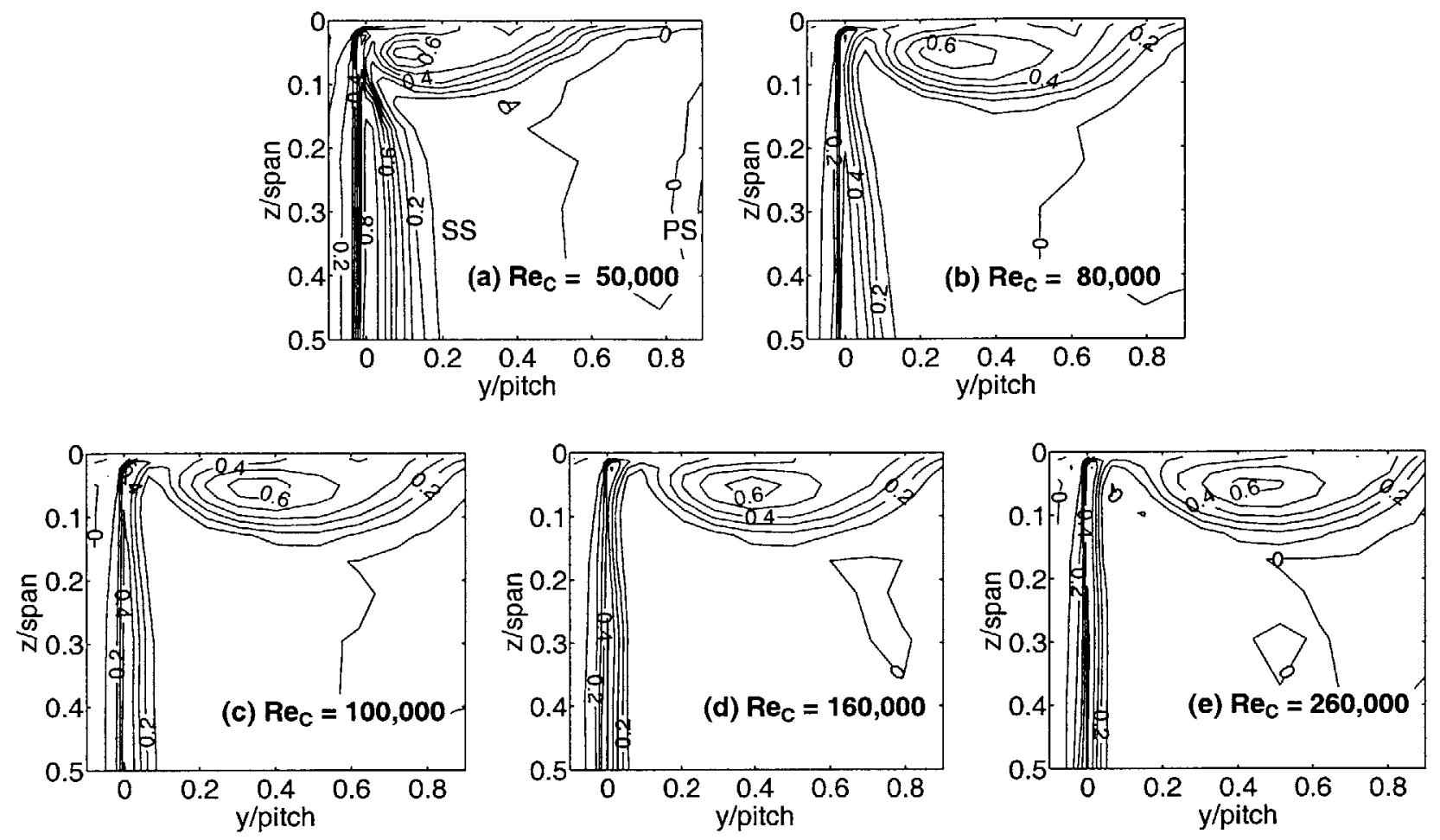

Figure 3.1 Contours of total pressure loss coefficient with various Reynolds numbers. $3 \% \mathrm{C}$ tip clearance.

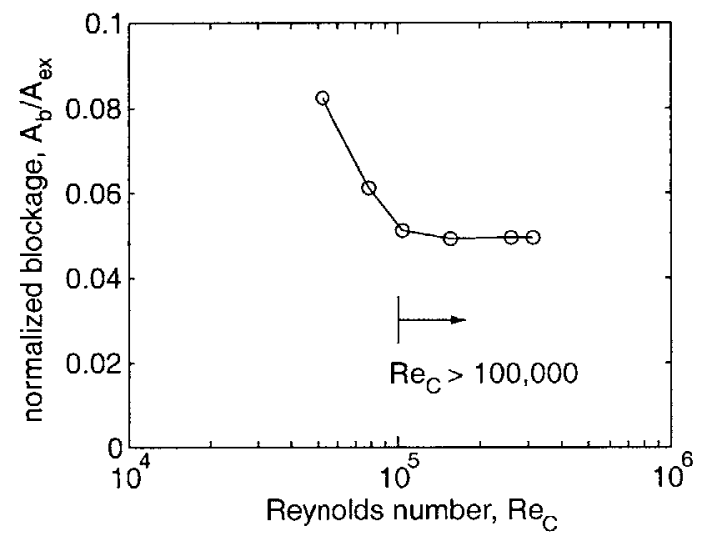

Figure 3.2 Reynolds number dependence of flow blockage. All quantitative measurements are done with $\operatorname{Re}_{C}$ above 100,000 .

\subsection{FLOW NEAR MID-SPAN}

The cascade is tuned by adjusting the upstream sidewall bleed ports and the downstream sidewall angle to achieve near blade-to-blade periodicity and design loading. The measured static pressure along mid-span on the center blade 
is compared with a computed result using MISES [3.1] as shown in Figure 3.3. The error bar in the figure is the uncertainty in $C_{P}$ measurement with $95 \%$ confidence. There is good agreement between measurements and MISES prediction, which was run at $\operatorname{Re}_{C}=350,000$ with turbulent boundary layers, confirming that the cascade has been set up correctly according to the design. The difference in the mid-span static pressure distribution with $0 \% \mathrm{C}$ and $3 \% \mathrm{C}$ clearances is less than the measurement uncertainty.

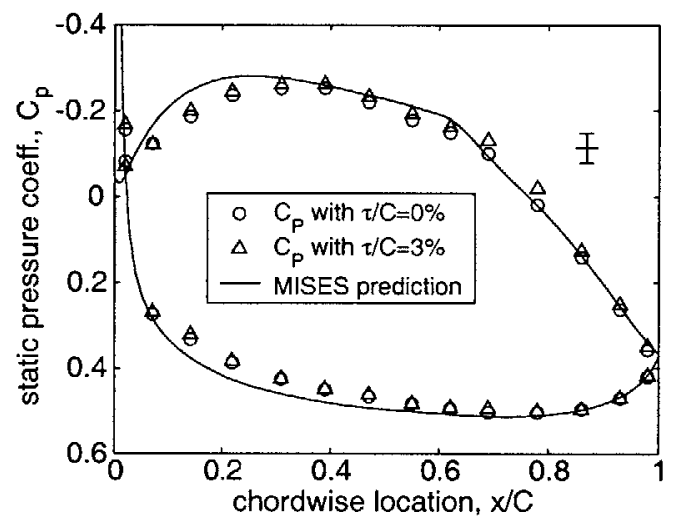

Figure 3.3 Mid-span $C_{P}$ distribution. Comaprison between measurements on center blade and CFD prediction using MISES [3.1].

\subsection{SURVEY OF TOTAL PRESSURE LOSS COEFFICIENT}

Figure 3.4(a) - (e) show the contour plots of the total pressure loss coefficient, $\omega$ for various tip clearance sizes; $\tau=$ $0,1,2,3$, and $4 \% C$ respectively. The contour plot with $0 \% C$ clearance (Figure 3.4(a)) shows the endwall boundary layer remains close to the casing wall. As the tip clearance increases, the area of the low total pressure (or momentum) fluid in the endwall region increases and the low total pressure area extends from the suction surface as well as the endwall. The pitchwise location of the tip clearance vortex core remains approximatcly constant regardless of tip clearance gap as was shown by Chen et al. [3.2]. 

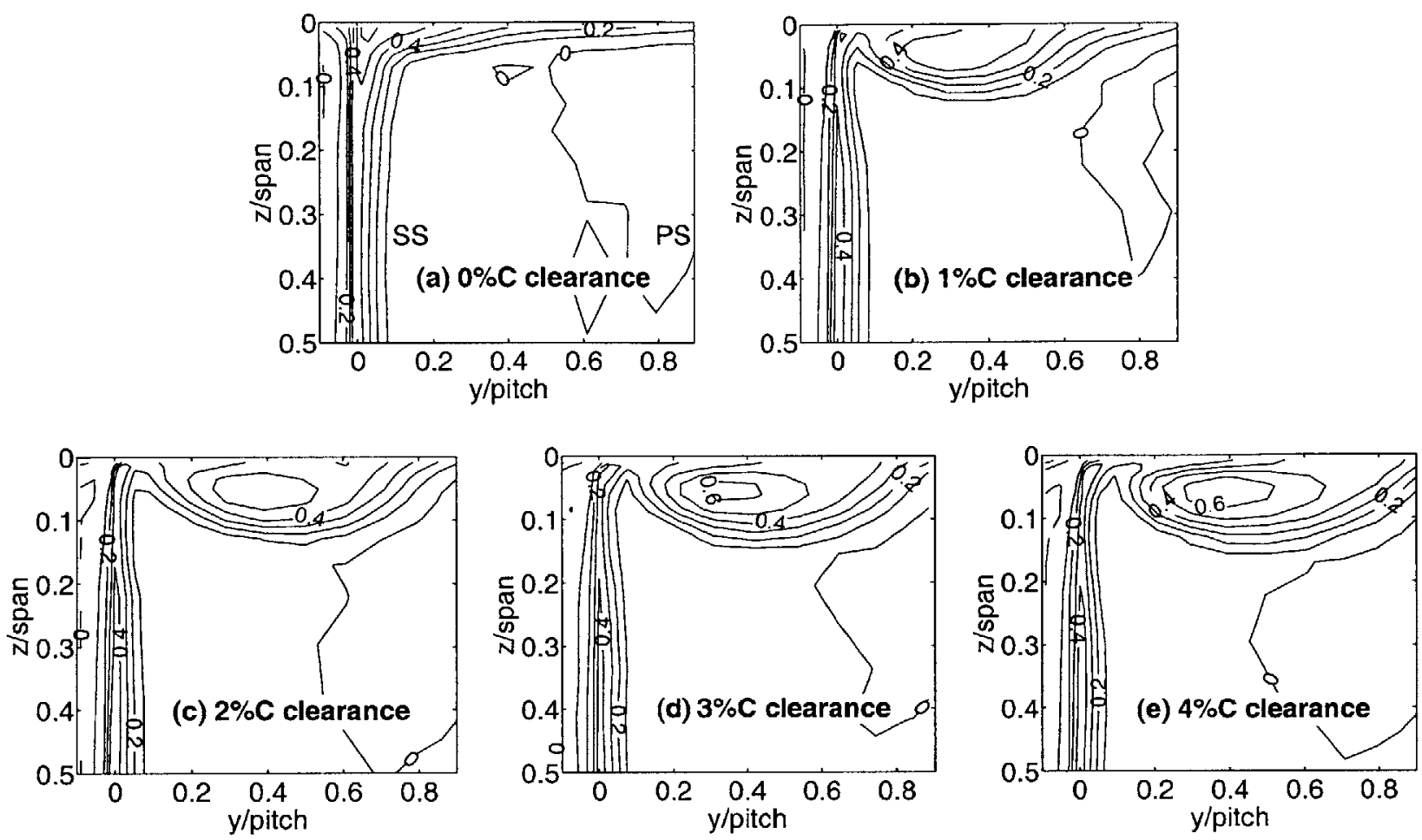

Figure 3.4 Contours of total pressure loss coefficient, $\omega$ measured $5 \% \mathrm{C}$ downstream of trailing edge plane.

\subsection{EFFECT OF TIP CLEARANCE SIZE ON PERFORMANCE}

The effect of tip clearance size on pressure rise and loss is quantified by measuring the endwall blockage, static pressure rise, mass-averaged total pressure loss, and stream thrust-averaged total pressure loss as functions of tip clearance size.

Figure 3.5 shows the endwall flow blockage defined as:

$$
A_{b}=\int\left(1-\frac{U_{x}}{U_{x, \text { edge }}}\right) d A
$$

vs. tip clearance. The integration is done only over the defect region, whose edge is defined as $\omega=0.05$ (slightly above the uncertainty of $\omega$, which is \pm 0.034 ) in this study to eliminate erroneous defect regions from the integration. In computing the blockage from the total pressure loss coefficient survey data in Figure 3.4, it is assumed that the survey plane static pressure is uniform and equal to the exit static pressure measured with the taps on the casing wall and the flow is unidirectional in the direction of the mass-averaged exit flow angle near mid-span predicted by MISES. The axial velocity in the plane is then: 


$$
U_{x}=U_{\infty} \sqrt{\left(1-\omega-C_{P, e x}\right)} \cos \beta_{e x}
$$

The endwall blockage is computed by subtracting the blockage associated with the blade surface boundary layers from the total blockage:

$$
A_{b, \text { ew }}=A_{b}-\left(\delta^{*} \times \frac{h}{2}\right)
$$

where $\delta^{*}$ is the displacement thickness of the blade wake near mid-span and $h$ is the length of the blade span. The endwall blockage shown in Figure 3.5 is normalized by the exit area, $A_{e x}=s \times h$. The uncertainty introduced by the assumption and the measurement error is estimated for 3\%C clearance and the uncertainty bound with $95 \%$ confidence level is shown in Figure 3.5. The solid line is the least-square fit of the data points. The endwall blockage increases approximately linearly with tip clearance (Khalid et al. [3.3]).

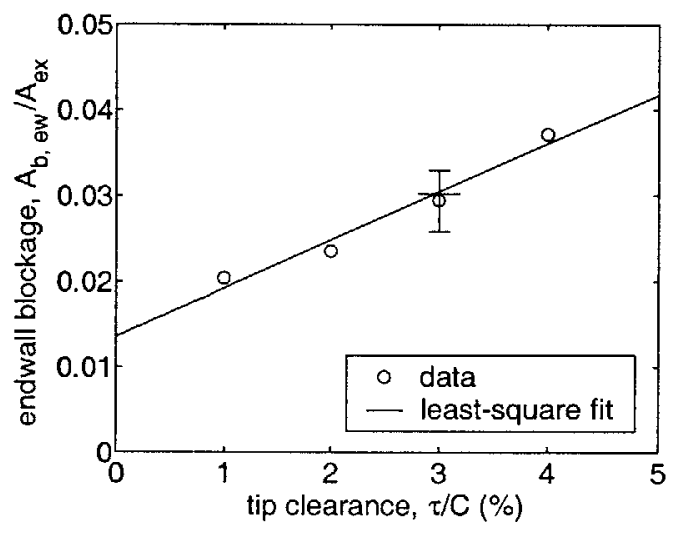

Figure 3.5 Endwall blockage measured 5\%C downstream of trailing edge plane vs. tip clearance size.

Figure 3.6 shows the exit static pressure measured using the six pressure taps on the casing wall $5 \% C$ downstream of the trailing edge plane. Again the solid line is the least-square fit of the data. The dashed line is cstimation based on the Bernoulli equation, which relates the static pressure rise to the effective area ratio determined by the least-square fit of the endwall blockage in Figure 3.5:

$$
C_{P, e x}=1-\left\{1-\left(C_{P, e x}\right)_{\tau=0}\right\} \cdot\left\{1-\left(\frac{A_{b, e w}}{A_{e x}}\right)_{\tau=0}\right\}^{2} /\left(1-\frac{A_{b, e w}}{A_{e x}}\right)^{2}
$$

The slopes of the solid and dashed lines are approximately the same emphasizing the importance of endwall blockage on the static pressure rise. However, the uncertainty in the measurement is larger than the quantities of interest as indicated by the error bar in Figure 3.6. Therefore, the endwall blockage is chosen over the exit static pressure as a measure of pressure rise capability. 


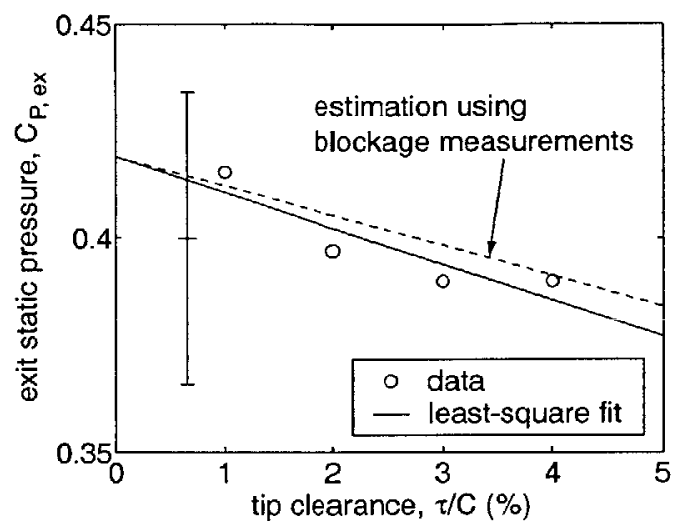

Figure 3.6 Static pressure measured 5\%C downstream of trailing edge plane using six pressure taps on endwall.

The mass-averaged endwall total pressure loss coefficient is computed from the Kiel probe measurements under the same assumptions as in the blockage calculation. Figure 3.7 shows the mass-averaged endwall total pressure loss coefficient, which is a measure of the total pressure loss incurred from the far upstream reference state to the averaging plane, plotted for various tip clearance sizes. The mass-averaged total pressure loss coefficient is defined as follows:

$$
\bar{\omega}^{m}=\frac{\int \omega U_{x} d A}{\int U_{x} d A}
$$

The two-dimensional profile loss associated with blade wake is subtracted from the mass-averaged total pressure loss coefficient, $\bar{\omega}^{m}$ to get the mass-averaged endwall total pressure loss coefficient, $\bar{\omega}_{e w}^{m}$. Again the estimated uncertainty with $95 \%$ confidence level is shown in the figure with an error bar. It is recognized that the loss due to the tip clearance flow increases linearly with tip clearance for a fixed mid-span loading (Storer and Cumpsty [3.4]). The solid line in Figure 3.7 is a least-square fit of the data. 


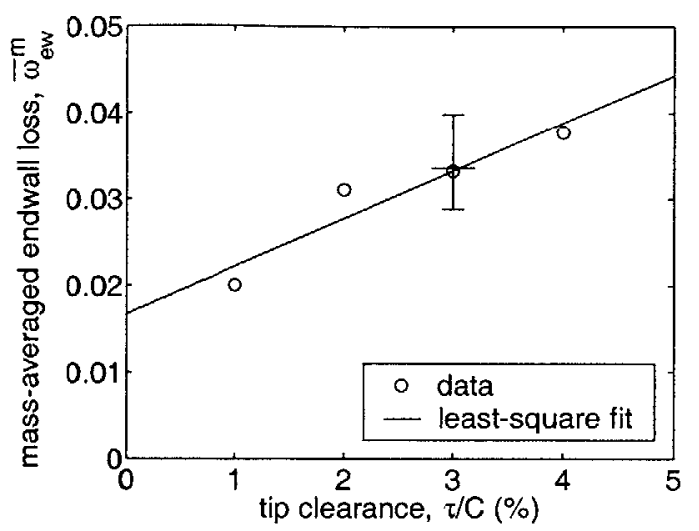

Figure 3.7 Mass-averaged endwall total pressure loss coefficient vs. tip clearance size.

Figure 3.8 shows the stream thrust-averaged (or fully mixed-out) endwall total pressure loss coefficient vs. tip clearance. The stream thrust-averaged endwall loss is calculated by hypothetically mixing out the flow in the surveyed area (excluding the blade wake) to a uniform state in a constant area duct conserving mass and momentum. The flow field is again estimated under the same assumptions made in the blockage calculation. Note that the stream thrust-averaged endwall loss is larger than the mass-averaged endwall loss shown in Figure 3.7 since it includes the additional mixed-out loss from the surveyed plane to the hypothetical uniform state. Storer and Cumpsty [3.4] proposed an analytical model based on a control volume analysis to predict the total pressure loss due to the tip leakage flow. The model is applied to the current cascade and the result is shown in Figure 3.8. Because the model only accounts for the loss due to the tip leakage flow, the intercept of the dashed line with the ordinate is anchored to the intercept of the least-square fit line. Measurement and the calculation are in good agreement justifying the assumptions made in the calculation of the stream thrust-averaged loss from the total pressure measurements.

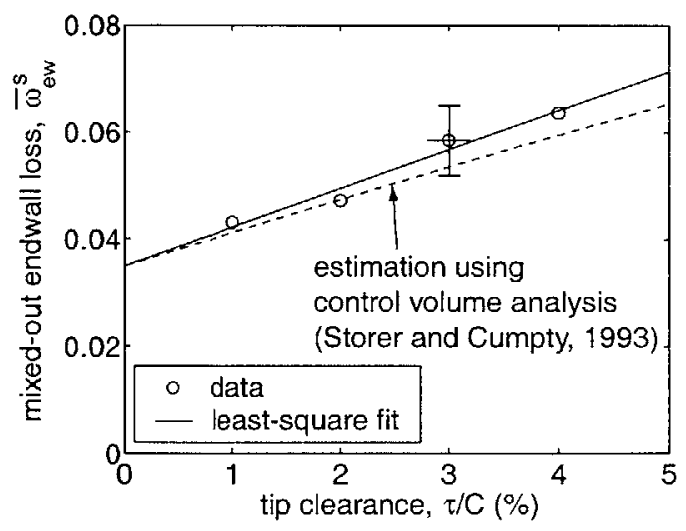

Figure 3.8 Stream thrust-averaged total pressure loss coefficient vs. tip clearance size. 
In summary, tip clearance-related endwall blockage, mass-averaged loss, and stream thrust-averaged (mixed out) loss all increase linearly with tip clearance; for a fixed mid-span loading the leakage flow rate is proportional to the tip clearance size (Storer and Cumpsty [3.4]). Figure 3.5 through Figure 3.8 are baseline results to which those from the flow control experiments can be compared to identify the process responsible for the changes in flow blockage with actuation.

\subsection{EFFECT OF NON-PERIODIC ENDWALL BLOCKAGE}

We only used one set of actuators to affect the endwall flow of the third passage from the bottom in Figure 2.2. To examine the effect of blade-to-blade non-periodicity the tip clearances of the three central blades were set to be different from one another. Non-uniform tip clearances would generate blade-to-blade non-periodicity, as the endwall blockage in each blade passage can be different from the other. Two cases have been tested. In the first case, the tip clearance of the center blade is set to $1 \% C$ and those of two adjacent blades are set to $3 \% C$. Measured blockage and loss of the third blade passage are compared with the case wherein all three central blades have uniform tip clearance of $1 \% C$. In the second case, the tip clearance of the center blade is set to $4 \% C$ and those of two adjacent blades are set to $2 \% C$. Measured blockage and loss of the third blade passage are now compared with the case wherein all three central blades have uniform tip clearance of $4 \% C$. The results of the above two cases are shown in Figure 3.9. Both cases match the uniform tip clearance case within the uncertainty of the measurements. As shown in the following chapter, the difference in the measurements due to non-periodic endwall blockage is negligible compared to the values of interest, i.e. changes that the actuation brings about. In summary, $50 \sim 70 \%$ blade-to-blade variations of the tip clearance size and related blockage and loss do not affect the measurement significantly. The results suggest that the effect of the tip clearance is limited within one blade pitch (or spacing) in the current cascade. 


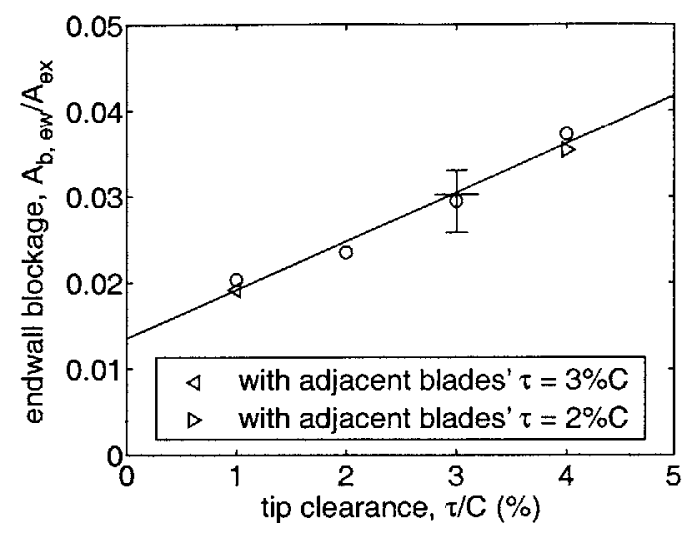

(a)

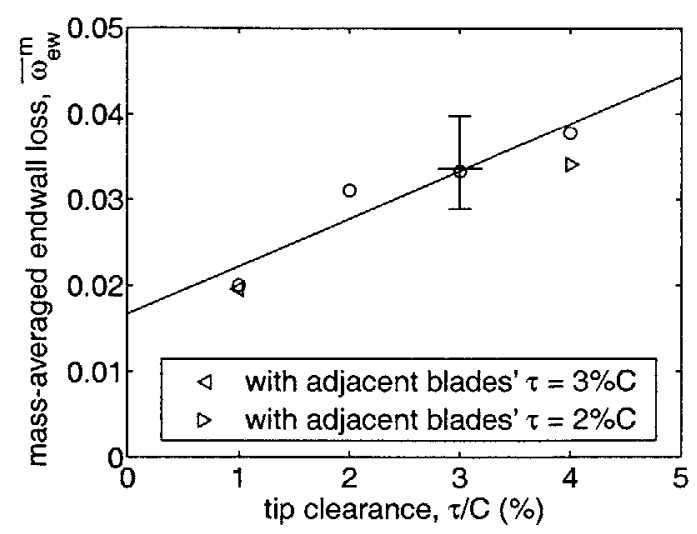

(b)

Figure 3.9 Measurements with non-periodic tip clearances compared with measurements with uniform tip clearances: (a) endwall blockage; (b) mass-averaged endwall loss.

\section{REFERENCES}

[3.1] Drela, M. and Youngren, H., "A User's Guide to MISES 2.1", MIT Computational Aerospace Science Laboratory, 1995.

[3.2] Chen, G. T., Greitzer, E. M., Tan, C. S., and Marble, F. E., "Similarity Analysis of Compressor Tip Clearance Flow Structure", ASME Paper No. 90-GT-153.

[3.3] Khalid, S. A., Khalsa, A. S., Waitz, I. A., Tan, C. S., Greitzer, E. M., Cumpsty, N. A., Adamczyk, J. J., and Marble, F. E., "Endwall Blockage in Axial Compressors", ASME Paper No. 98-GT-188.

[3.4] Storer, J. A. and Cumpsty, N. A., "An Approximate Analysis and Prediction Method for Tip Clearance Loss in Axial Compressors", ASME Paper No. 93-GT-140. 


\section{CHAPTER 4 TIME-AVERAGED EFFECTS OF ACTUATION}

In this chapter, the effects of actuation on the time-averaged change in tip clearance-related blockage and loss are determined. Specifically, we examine the influence of four parameters: actuator type (NSJ, DSJ, or SDJ), pitchwise location of the actuator on the casing relative to blade tip location, amplitude of actuation, and frequency at which the actuation is applied. The parametric dependence of the tip clearance-related blockage and loss on the actuation amplitude and frequency is explored and discussed. The flow processes responsible for the observed change in the blockage and loss are also identified.

\subsection{NORMAL SYNTHETIC JET ACTUATOR}

Figure 4.1(b) and (c) show the contour plots of $\omega$ taken with two pitchwise locations of the NSJ actuator: (b) directly over the blade tip and (c) approximately over the vortex $\operatorname{core}^{* *}$ as indicated by the arrows. There is noticeable change in the contours for both locations compared to the baseline in (a). As shown in Figure 4.2, the exit static pressure measurement indicates an increase in $C_{P,}$ ex with NSJ actuation, although it is only qualitative due to the large uncertainty in the measurement. Quantitative assessments are discussed in the following.

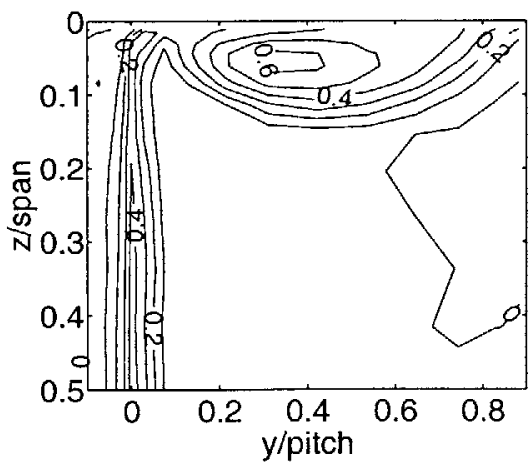

(a) baseline

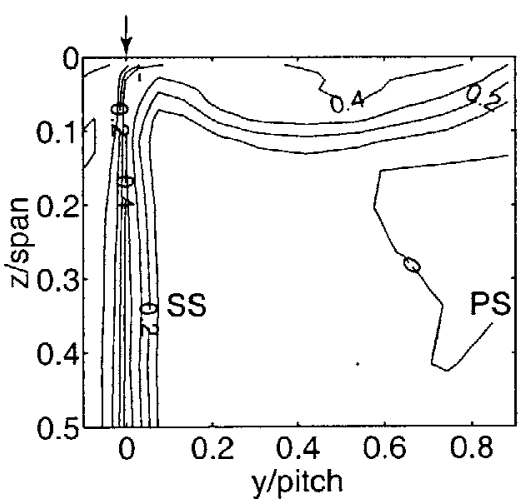

(b) NSJ over blade tip

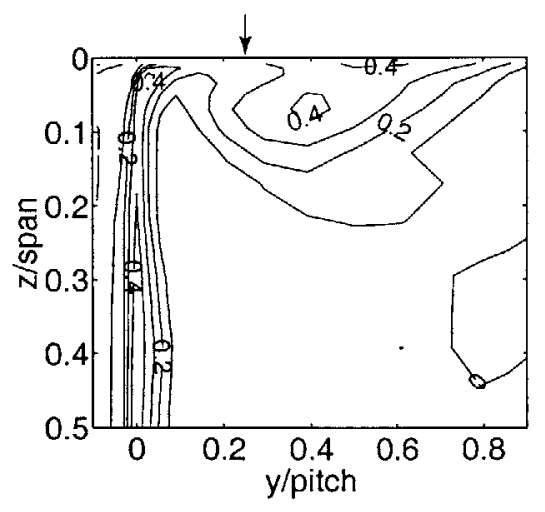

(c) NSJ over vortex core

Figure 4.1 Contours of $\omega$ measured $5 \% C$ downstream of trailing edge plane: (a) baseline without actuation, $\tau=3 \% C$; (b) NSJ directly over blade tip (at $0 \%$ pitch), $C_{\mu_{i}}=0.88, F_{C}^{+}=1.0 ;$ (c) NSJ over vortex core (at $25 \%$ pitch $), C_{\mu, \tau}=0.88, F_{C}^{+}=1.0$. Arrows indicate pitchwise locations of NSJ slits.

\footnotetext{
"* The actuator covers $70 \% C$ from L.E. Therefore, the NSJ slits upstream of the survey plane are approximately over the vortex core, although the arrow projected onto the survey plane appears to be closer to SS than the core.
} 


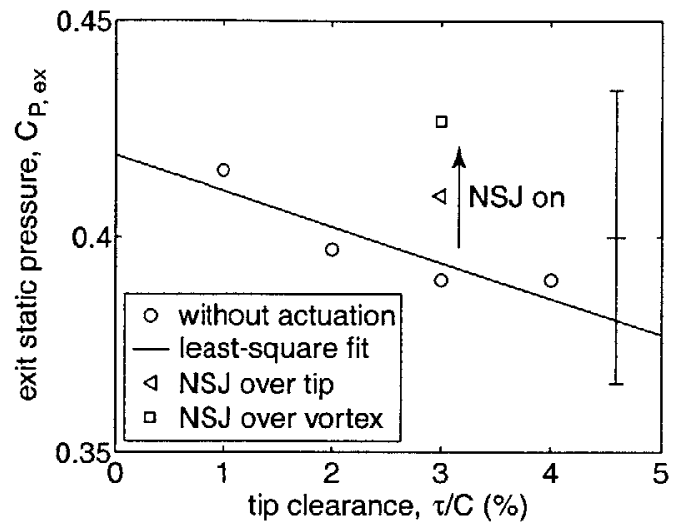

Figure 4.2 Exit static pressure with and without NSJ actuation. $\triangleleft$ : NSJ directly over blade tip (at 0\% pitch), $C_{\mu, \tau}=0.88, F_{C}^{+}=1.0 ; \square$ : NSJ over vortex core (at $25 \%$ pitch), $C_{\mu \tau}=0.88, F_{C}^{+}=1.0$.

\subsubsection{Scaling of NSJ Actuator Amplitude}

The momentum coefficient of the actuator (momentum of the actuator jet normalized by the momentum of the leakage flow) is used as a measure of the actuation amplitude. The leakage flow momentum is approximated based on far upstream flow velocity. Although the magnitude of the leakage jet velocity varies over the blade chord, it scales with $U_{\infty}$ and is well approximated by $U_{\infty}$ for different tip clearance sizes as shown in Figure 4.3. The magnitude of the leakage jet velocity in Figure 4.3 has been estimated from the static pressure measurement near the blade tip using a model given by Rains [4.1]:

$$
U_{L}=\sqrt{\frac{2\left(P_{t, \infty}-P_{S S, t i p}\right)}{\rho}}=U_{\infty} \sqrt{1-C_{P, S S, t i p}}
$$

where $P_{S S, \text { tip }}$ is the static pressure on the suction surface near the blade tip ( $1.5 \% C$ from the tip). This expression is based on the Bernoulli equation assuming ideal flow without loss across the tip. Storer and Cumpsty [4.2] showed that its use is in good agreement with their tip leakage flow measurements. The momentum coefficient is thus defined as:

$$
C_{\mu, \tau}=\frac{\rho U_{J, p e a k}^{2} A_{J}}{\rho U_{\infty}^{2} \tau C} .
$$




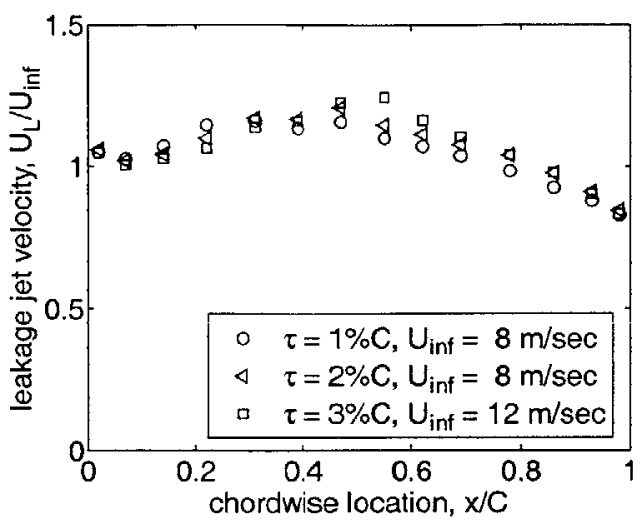

Figure 4.3 Magnitude of leakage jet velocity leaving tip clearance.

Figure 4.4 shows the tip clearance-related blockage versus amplitude of actuation for two tip clearances. The NSJ actuator is placed at a pitchwise location $0.25 \mathrm{~s}(25 \%$ pitch) from the camberline of the center blade so that it is approximately over the vortex core. The blockage (mostly dominated by that associated with tip leakage flow) has been normalized by the baseline blockage of each clearance. The data set collapses onto a single curve with the implication that the actuator momentum scales with the leakage flow momentum as expected. Most of the tip clearance-related blockage can be eliminated using the NSJ actuator at $C_{\mu, \tau}$ of approximately 1.5 .

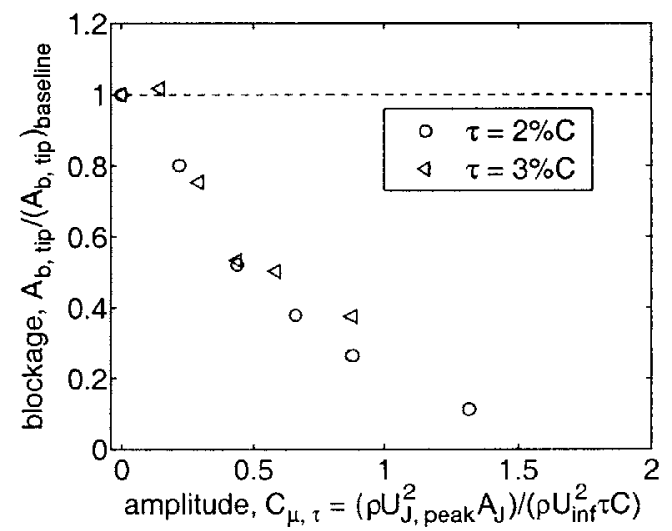

Figure 4.4 Tip clearance-related blockage vs. NSJ actuator amplitude. Actuator near vortex core. $F_{C}^{+}=1.0$.

The $\omega$ contour plots for two data points in Figure 4.4 with $2 \% C$ clearance are shown in Figure $4.5(\mathrm{~b})$ and (c) along with that for the baseline in Figure 4.5(a). In Figure 4.5(b), $C_{\mu, \tau}=0.44$ and in Figure 4.5(c), $C_{\mu, \tau}=1.32$. The size of the region with low total pressure increases while the depth of the $P_{l}$ defect decreases with NSJ actuation, suggesting that the defect region becomes more uniform due to the NSJ actuation. 


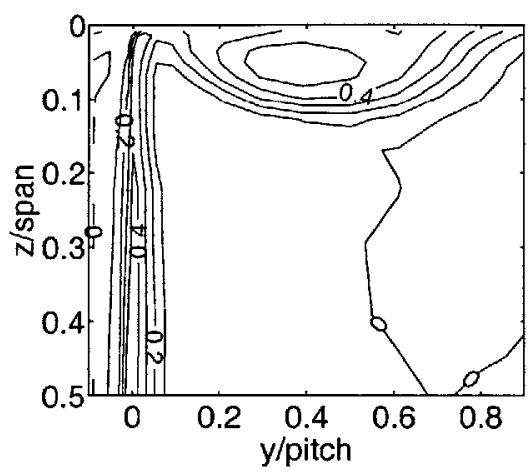

(a) baseline

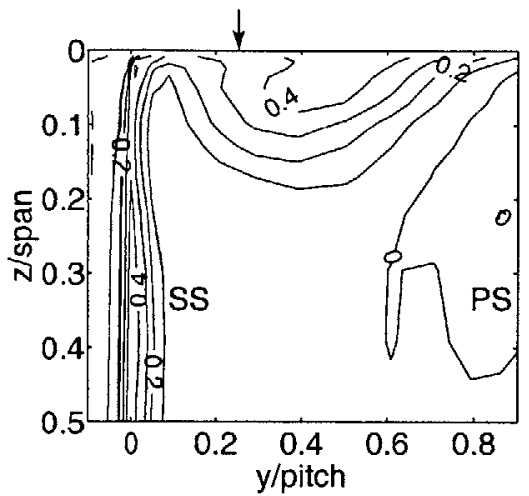

(b) $C_{\mu, \tau}=0.44$

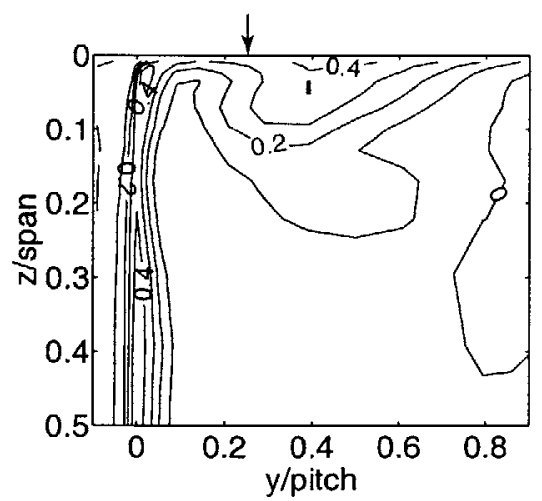

(c) $C_{\mu \tau}=1.32$

Figure 4.5 Contours of $\omega$ measured $5 \% C$ downstream of trailing edge plane: (a) baseline without actuation, $\tau=2 \% C$; (b) NSJ over vortex core (at $25 \%$ pitch), $C_{\mu_{+} \tau}=0.44, F_{C}^{+}=1.0 ;$ (c) $\mathrm{NSJ}$ over vortex core, $C_{\mu, \tau}=1.32$, $\mathrm{F}_{\mathrm{C}}^{+}=\mathbf{1 . 0}$.

The endwall blockages taken with two upstream velocities are plotted against the actuator amplitude as shown in Figure 4.6. The horizontal lines are the baseline blockages without actuation at each clearance determined from Figure 3.5. The two data sets form a single trend when plotted non-dimensioanlly against $C_{\mu, \tau}$ showing that the definition of $C_{\mu, \tau}$ appropriately reflects the dependence of the blockage reduction on the far upstream velocity.

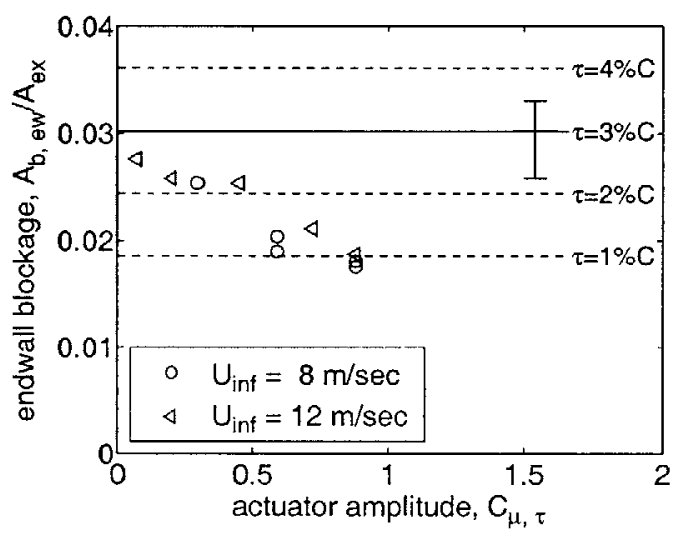

Figure 4.6 Endwall blockage vs. $C_{\mu, \tau}$ of NSJ direcly over blade tip. Data sets taken for two upstream velocities. $\tau=3 \% C, F_{C}{ }^{+}=1.0$.

\subsubsection{Dependence on Forcing Frequency of NSJ}

The blockage reduction achieved with the use of NSJ exhibits considerable sensitivity to the forcing frequency. Thus we need to first identify/define a reduced frequency parameter, which scales this sensitivity. It was suggested that the observed reduction in blockage is associated with the instability of the shear layer developed at the blade tip as in the mixing enhancement of two-dimensional wall bounded jets (Katz et al. [4.3] and Tsuji et al. [4.4]). If that is 
the case, the frequency should scale with the height of the wall jet or the tip clearance size $\tau$. Figure 4.7 shows the frequency response of the endwall blockage taken with NSJ actuator placed at $25 \%$ pitch. There are two data sets with two tip clearance sizes. In Figure 4.7(a), the tip clearance size $\tau$ is used to non-dimensionalize the forcing frequency as follows:

$$
\beta_{\tau}=\frac{2 \pi f \tau}{U_{\infty}}
$$

The blockage is the most responsive when forced at $\beta_{\tau}=0.05 \sim 0.25$. However, the troughs of the endwall blockage for the two data sets do not line up vertically as indicated with the dashed line, implying that the flow mechanism is not related to the shear layer instability.

In Figure 4.7(b), the blade chord length $C$ is used instead of $\tau$ to non-dimensionalize the forcing frequency as follows $^{\text {†t: }}$

$$
F_{C}^{+}=\frac{f C}{U_{\infty}}
$$

The blockage is the most responsive when forced at a reduced frequency $F_{C}{ }^{+}$of about 0.75 and the troughs of the endwall blockage for the two data sets both nearly line up vertically.

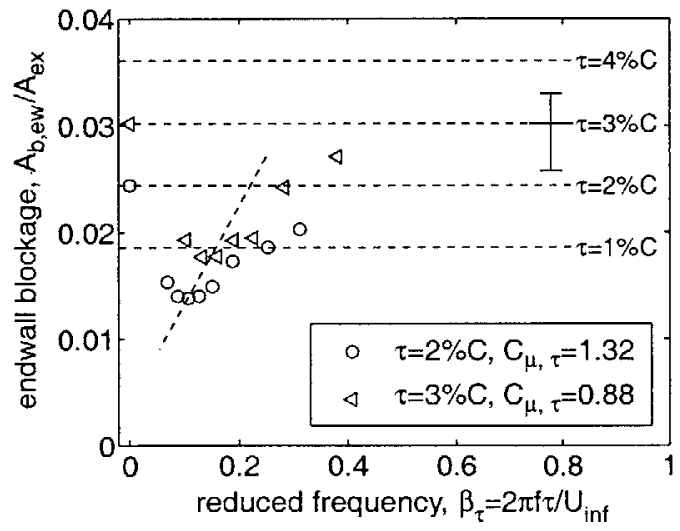

(a)

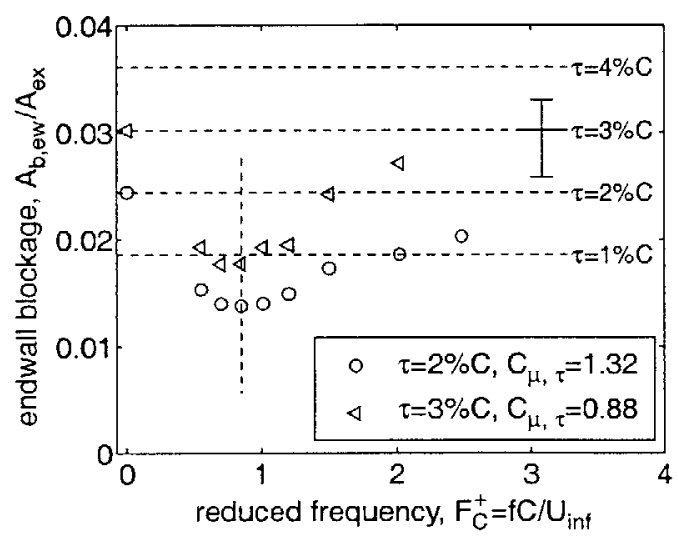

(b)

Figure 4.7 Frequency dependence of blockage reduction with NSJ actuator over vortex core: (a) using clearance size $\tau$; (b) using blade chord $C$ as length scale.

${ }^{\dagger \dagger}$ The factor of $2 \pi$ only appears in the definition of $\beta_{\tau}$. The above definition of $\beta_{\tau}$ is typically used in the wall jet stability community, while that of $F_{C}{ }^{+}$is in the flow separation control community. 
Figure 4.8 shows similar data sets taken with the NSJ actuator acting directly over the blade tip, for three tip clearances. Again, the blockage troughs line up vertically at a reduced frequency $F_{C}{ }^{+}$of about 1.0 when the blade chord length is used to non-dimensionalize the forcing frequency.

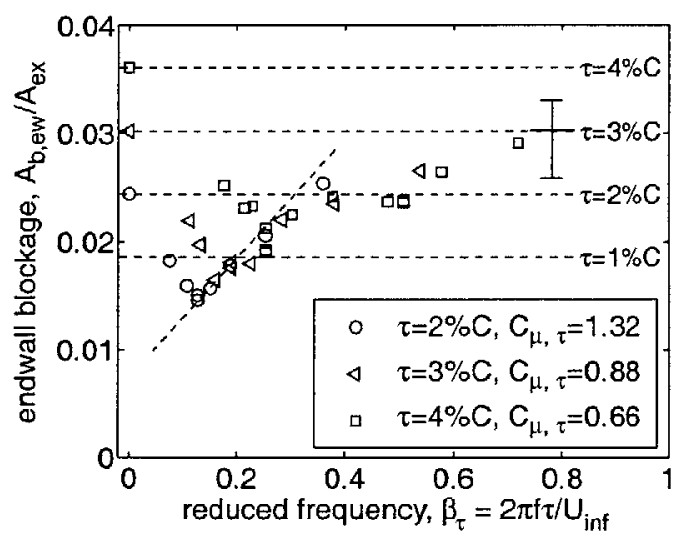

(a)

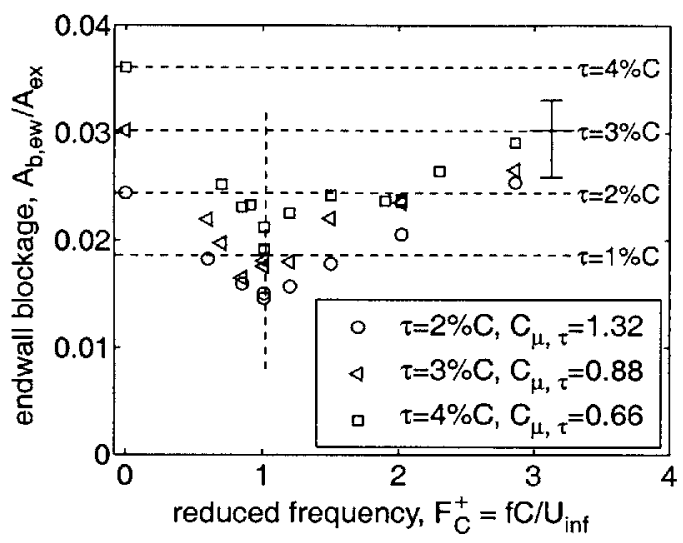

(b)

Figure 4.8 Frequency dependence of blockage reduction with NSJ actuator over blade tip: (a) using clearance size $\tau$; (b) using blade chord $C$ as length scale.

Unlike the tip clearance size, the blade chord length was not varied to determine its relevance in defining $F_{C}{ }^{+}$. As will be presented in the next chapter, a model-based approach is taken to justify the use of blade chord as the length scale and to explain the flow mechanism that results in the blockage reduction. On a tentative basis, blade chord length will be used as the length scale of the problem for the rest of this chapter.

\subsubsection{Dependence on Pitchwise Location of NSJ}

As mentioned in subsection 2.3.1, the casing wall with the actuator is adjustable in the pitchwise direction and the survey of total pressure loss coefficient was carried out with various pitchwise NSJ slit locations. Figure 4.9 shows the dependence of the blockage reduction on the pitchwise location of the NSJ actuator with $3 \% C$ clearance. The baseline blockage without actuation is indicated with a solid horizontal line. The locations of the blades are indicated in the bottom of the figure. There are two local optimum pitchwise locations for the blockage reduction: one right over the blade tip and the other over the vortex core. At these optimum pitchwise locations, the tip clearance-related blockage is reduced by about $66 \%$, which is equivalent to the baseline case with $1 \% C$ clearance. The actuator amplitude and frequency were fixed at $C_{\mu, \tau}=0.88$ and $F_{C}{ }^{+}=1.0$. The bulk flow process responsible for the blockage reduction will be discussed in subsection 4.1.6. 


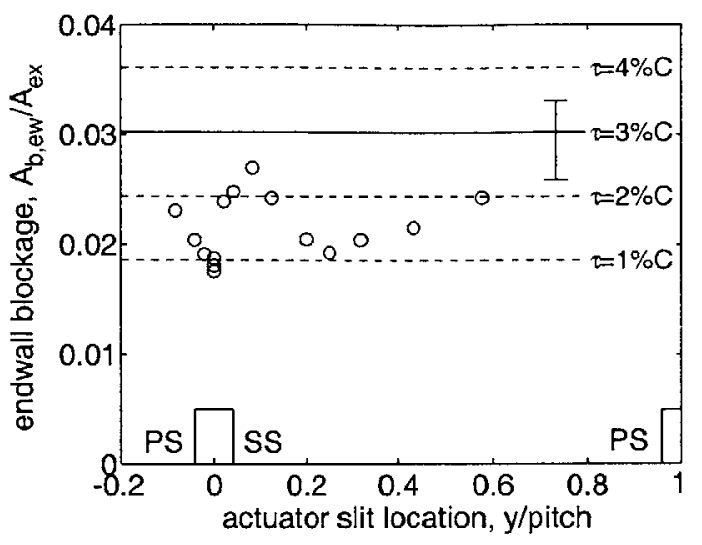

Figure 4.9 Endwall blockage vs. pitchwise location of NSJ actuator. $\tau C=3 \%, C_{\mu, \tau}=0.88$ and $F_{C}{ }^{+}=1.0$.

Total pressure loss coefficient variation with pitchwise location of the NSJ is shown in Figure 4.10. Figure 4.10(a) shows that the mass-averaged loss increases with actuation compared to the baseline (the solid horizontal line) except for the data with the actuator over the blade tip where it remains more or less the same. The massaveraged loss is largest at around 0.25 pitch where the blockage reduction is locally largest as shown in Figure 4.9. Tip clearance-related loss at 0.25 pitch is increased by roughly $83 \%$ to the same level as the extrapolated baseline case with $5.5 \% C$ clearance. Figure 4.10 (b) shows the stream thrust-averaged (i.e. mixed-out) loss vs. the pitchwise location of the NSJ actuator. The stream thrust-averaged loss remains more or less the same as the baseline when the actuator is away from the blade tip. However, when the actuator is right over the blade, the tip clearance-related stream thrust-averaged loss is reduced by about $33 \%$ so that it is the same as the baseline case with $2 \% C$ clearance.

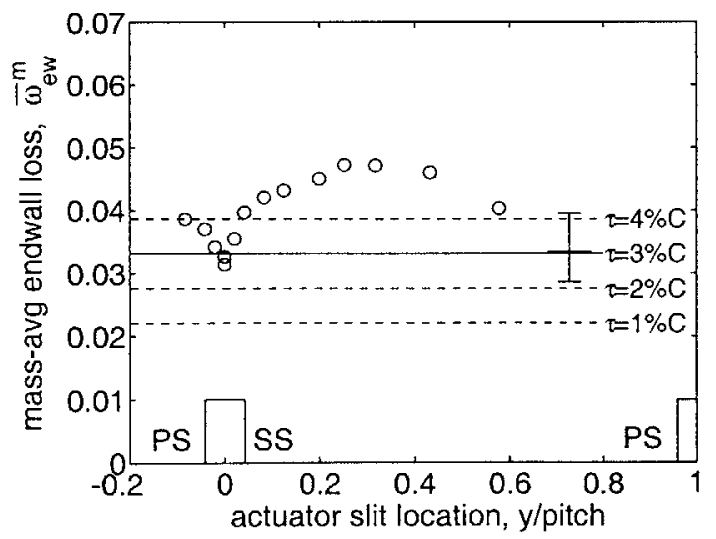

(a)

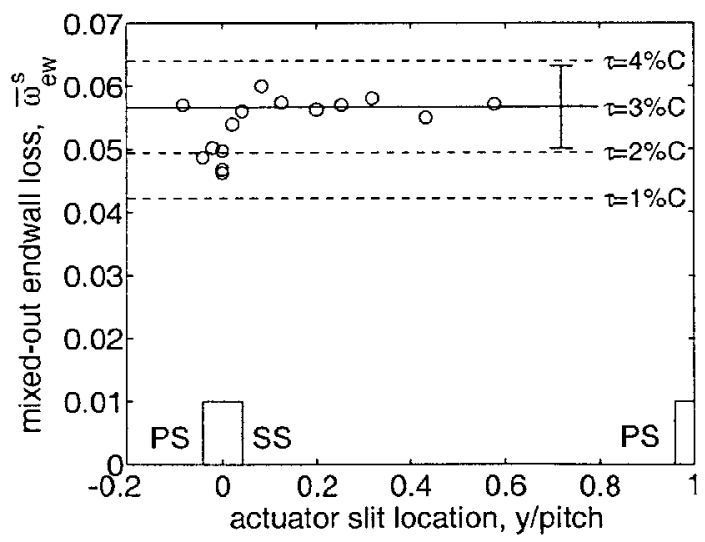

(b)

Figure 4.10 Endwall total pressure loss coefficient vs. pitchwise location of NSJ actuator. $t C=3 \%, C_{\mu} \tau=$ 0.88 and $F_{C}{ }^{+}=1.0$ : (a) mass-averaged $\omega$, (b) stream thrust-averaged (or fully mixed-out) $\omega$ 


\subsubsection{Contribution of Each NSJ Actuator}

As mentioned earlier, there are three independent NSJ actuators inline as shown in Figure 2.2 and Figure 2.4. The endwall blockage has been measured with one NSJ actuator at a time to examine the contribution of each actuator ${ }^{\ddagger \ddagger}$, i.e. to study whether there is an axial location wherein the NSJ actuation is more effective. Figure 4.11 shows the endwall blockage plotted against the actuator amplitude for NSJ placed over the blade tip. Three chordwise locations of the NSJ considered in this study are equally effective in blockage reduction. In other words, the blockage reduction with one actuator is almost the same regardless of the actuator and the amount of blockage reduction is nearly the same as the case with all three actuators at the same total $C_{\mu \tau}$, although the actuation jet velocities are different. For the same total $C_{\mu, \tau}$ of 0.29 , the jet area $A_{J}$ of one actuator case is $1 / 3$ of the case with all three actuators and the peak jet velocity $U_{J, \text { peak }}$ is $3^{1 / 2}$ (or 1.73 ) times that of the case with all three actuators. This result also points out the relevance of $C_{\mu, \tau}$ in the blockage reduction with the use of NSJ.

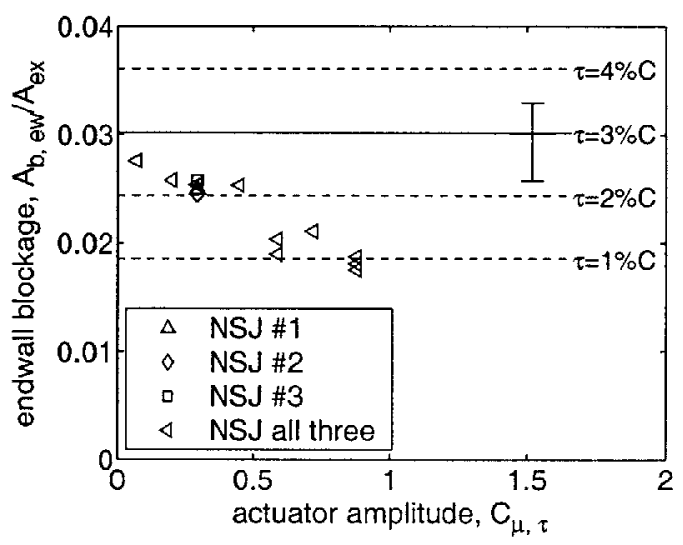

Figure 4.11 Contribution of each NSJ actuator placed directly over blade tip. Amplitude is total $C_{\mu, \tau}$ from all working actuators. All data taken with $F_{C}{ }^{+}=1.0$ and $\tau / C=3 \%$.

\subsubsection{Flow Visualization}

To examine whether there is a change in the level of mixing between the lcakage jet and the main flow with the use of NSJ actuation, flow visualization has been carried out (A detailed description of the visualization setup is in subsection 2.2.3). To capture identifiable image with given settings, the Reynolds number had to be minimized and was set at $R e_{C}=80,000\left(U_{\infty}=6 \mathrm{~m} / \mathrm{s}\right)$. Two of the resulting images are shown in Figure 4.12. The top of each image

\footnotetext{
\# The NSJ near L.E. is NSJ \#1, and the next one downstream is NSJ \#2, and so on.
} 
is the casing wall and the left-hand side is the suction surface of the blade. Each pixel in the digitized image has an intensity $I$ which spans from 0 (white) to 1 (black or smoke). The contour lines of $I$ are computer-processed and plotted on top of the images. Figure 4.12(a) is the baseline image without actuation. The leakage jet comes in from the clearance gap in the upper-left corner rolling up into a vortex. Most of the smoke is collected in the core region of the vortex. Figure 4.12(b) is with actuation over blade tip at $F_{C}{ }^{+}=1.0$ and $C_{\mu, \tau}=0.88$. Note that the forcing frequency is the optimum for the blockage reduction as shown in Figure 4.8(b). The smoke is spread out more uniformly compared to that of the baseline in Figure 4.12(a); this has the implication that NSJ actuation promotes mixing between the smoke-tagged leakage flow (black) and the untagged blade passage flow (white).

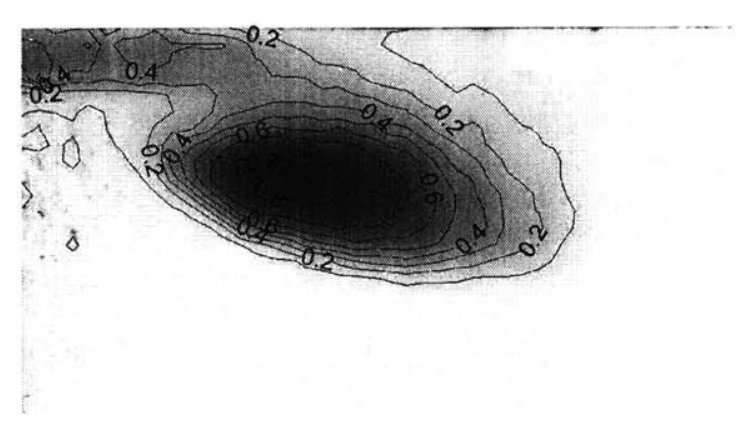

(a)

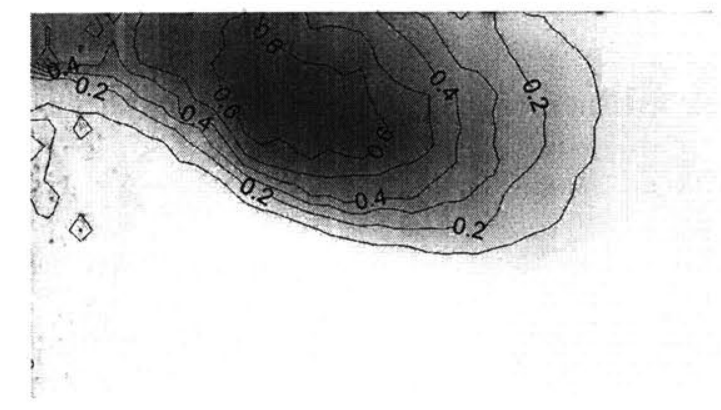

(b)

Figure 4.12 Images from flow visualization and computer-processed intensity contour lines: (a) baseline without actuation $(\tau=3 \% C)$; (b) with NSJ actuation over blade tip $\left(F_{C}{ }^{+}=1.0\right.$ and $\left.C_{\mu, \tau}=0.88\right)$.

The intensity in the image is an indication of the concentration of the smoke particles. Since the mass diffusion per unit area is proportional to the concentration gradient, the average magnitude of the concentration (or the intensity in the image) gradient is a good measure of the level of diffusion and hence the level of mixing. The intensity gradient would become zero, if the smoke (or the leakage flow) were fully mixed with the core flow. To quantitatively compare the relative level of mixing at three forcing frequencies, the gradients of the intensity have been processed and the average of their magnitudes are plotted against the forcing frequency as shown in Figure 4.13. The average magnitude of the gradient $\overline{(|\nabla I|)}$ has a dependence on the forcing frequency with the smallest $\overline{(|\nabla I|)}$ at $F_{C}{ }^{+}=1.0$ among three forcing frequencies tested. Although the average intensity $\bar{I}$ of each image is within $\pm 2 \%$, there exists up to $30 \%$ reduction in $\overline{(|\nabla I|)}$, implying that the mixing is enhanced by actuation and is largest at near ${F_{C}}^{+}=1.0$. Enhanced mixing between the low momentum fluid in the vortex core and the external stream would reduce the streamwise velocity non-uniformity (and blockage). 


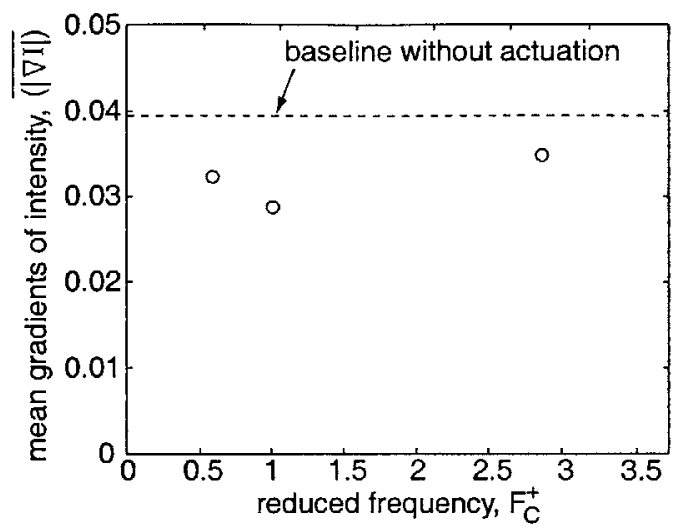

Figure 4.13 Average magnitude of gradients of intensity in flow visualization images indicating mixing level.

\subsubsection{Bulk Flow Process Associated with NSJ Actuation}

The dependence of the blockage reduction on the forcing frequency excludes momentum injection as a source because the same time-averaged momentum results in almost no change in blockage at frequencies far away from the optimum as shown in Figure 4.7 and Figure 4.8. Two possible bulk flow processes associated with the NSJ actuation can be suggested as responsible for the reduction in tip clearance-related blockage: leakage flow reduction and mixing enhancement. Kang et al. [4.5] showed that the NSJ actuation does not change the leakage flow rate when placed away from the blade tip. Blockage reduction with the actuator over the vortex core is thus attributed to the mixing enhancement. Mixing enhancement shortens the distance for mixing and consequently increases the mass-averaged loss, although the stream thrust-averaged loss is not changed (See Figure 4.10 at $y=0.25$ pitch).

The blockage reduction with the NSJ actuator over the blade tip is a combination of the two flow processes. As observed by Kang et al. [4.5] the NSJ actuator can reduce the amount of the leakage flow for a given pressure difference across the blade when it is placed over the blade tip. One can indirectly quantify the reduction in the leakage flow rate in the cascade experiment from the stream thrust-averaged loss measurements shown in Figure 4.10(b). As discussed above the mixing enhancement does not change the stream thrust-averaged loss. The reduction in the stream thrust-averaged loss with NSJ actuator over the blade tip is thus attributed to the reduction in the leakage flow rate. The model of Storer and Cumpsty [4.6] suggests that for fixed mid-span loading tip clearancerelated loss increases linearly with the leakage flow rate. The $33 \%$ reduction in the tip clearance-related stream thrust-averaged loss shown in Figure 4.10(b) leads one to conclude that the leakage flow rate is reduced by about $33 \%$ due to the NSJ actuation over the blade tip. The 33\% reduction in the leakage flow rate results in a $33 \%$ reduction of tip clearance-related blockage and loss, which are proportional to the leakage flow rate, as summarized 
in the second row of Table 4.1. About half of the blockage reduction is thus viewed as due to the leakage flow reduction.

As described in subsection 4.1.5, the NSJ actuator over the blade tip also brings about mixing enhancement and the other half of the blockage reduction is attributed to this effect. The net effects of the two flow processes on the tip clearance-related blockage, mass-averaged, and stream thrust-averaged losses are listed Table 4.1. The net effect on the mass-averaged loss remains the same as a result of the compensating effect of the tip leakage flow reduction and the mixing enhancement. In summary, both leakage flow reduction and mixing enhancement are responsible for the reduction in blockage with NSJ actuator over the blade tip and their contributions are roughly the same.

Table 4.1 Effects of each flow process and their combined effects on tip clearance-related blockage and loss explaining observations with NSJ actuator over blade tip.

\begin{tabular}{|l|ll|ll|ll|}
\hline & \multicolumn{2}{|l|}{ Blockage } & Mass-averaged loss & Fully mixed-out loss \\
\hline Leakage flow reduction & $\downarrow$ & Decrease by 33\% & $\downarrow$ & Decrease by 33\% & $\downarrow$ & Decrease by 33\% \\
\hline Mixing enhancement & $\downarrow$ & Decrease by 33\% & $\uparrow$ & Increase by 33\% & - & Same \\
\hline Net effect & $\downarrow \downarrow$ & Decrease by 66\% & - & Same & $\downarrow$ & Decrease by 33\% \\
\hline
\end{tabular}

\subsection{DIRECTED SYNTHETIC JET ACTUATOR}

Figure 4.14 shows a representative result of the DSJ actuation compared with the baseline. There are substantial changes in the endwall flow. The exit static pressure measurements indicate an increase in $C_{p \text {, ex }}$ with DSJ as shown in Figure 4.15 but due to the large uncertainty in the measurement, the comparison is only qualitative. 


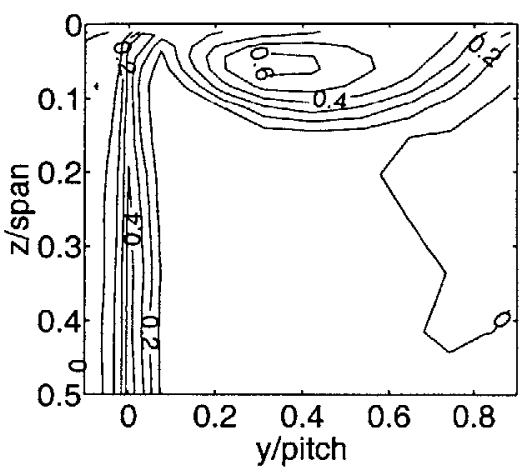

(a) baseline

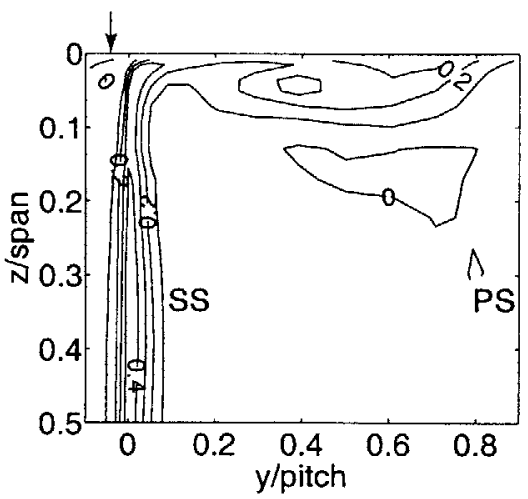

(b) DSJ over blade PS

Figure 4.14 Contours of $\omega$ measured $5 \% C$ downstream of trailing edge plane: (a) baseline without actuation, $\tau=3 \% C$; (b) DSJ directly over blade PS $(y=-0.04$ pitch $), C_{\mu, \tau}=0.88, F_{C}^{+}=1.0$. Arrows indicate locations of DSJ holes.

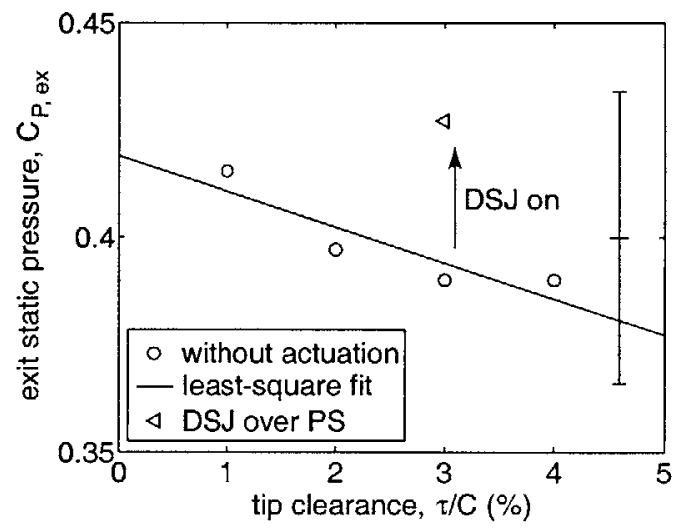

Figure 4.15 Exit static pressure with and without NSJ actuation. $\triangleleft:$ DSJ directly over blade PS (-0.04 pitch), $C_{\mu, \tau}=0.88, F_{C}^{+}=1.0$.

As with the cxperiments on the NSJ, the dependence on the amplitude, frequency, and pitchwise location of the DSJ actuator, and the contribution of each actuator (there are three actuators inline as shown in Figure 2.2 and Figure 2.4) were also determined. We also compare the use of SDJ (Steady Directed Jet) against DSJ to determine if steady injection of momentum with the same time-averaged value as that from DSJ yields similar benefit.

\subsubsection{Dependence on Amplitude of DSJ}

The response of the endwall blockage with the use of DSJ actuation is compared with that with the use of NSJ actuation in Figure 4.16. The endwall blockage is plotted against the actuator amplitude with each actuator placed at one of its most effective pitchwise locations for blockage reduction, i.e. for the case where the DSJ actuator is placed near the pressure side of the blade $(y=-0.04$ pitch $)$ and for the situation where the NSJ actuator is placed near 
the vortex core. The forcing frequency is $F_{C}{ }^{+}=1.0$ for both. The DSJ actuator is about twice as effective as the NSJ actuator in reducing the endwall blockage for a given amplitude $C_{\mu, \tau}$ and eliminates most of the endwall blockage, not only that associated with tip clearance flow but also that associated with the endwall boundary layer at $C_{\mu, \tau}$ of about 1.0.

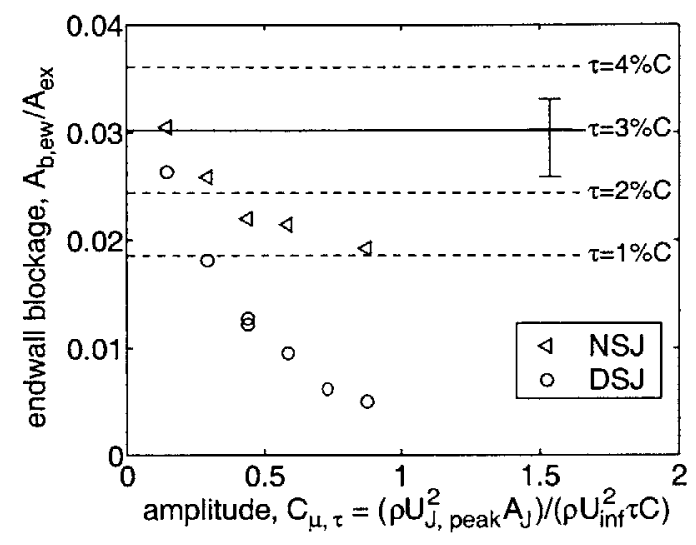

Figure 4.16 Comparison between DSJ (placed near pressure surface of blade) and NSJ (placed near vortex core). $\tau=3 \% C$. Both actuators at $F_{C}^{+}=1.0$.

\subsubsection{Dependence on Forcing Frequency of DSJ}

As shown in Figure 4.17, the blockage reduction with the DSJ is less sensitive to frequency than the NSJ. The variations in the data taken at two Reynolds numbers are less than the measurement uncertainty and no significant trend in the frequency response is observed. This suggests that the change in the flow process due to DSJ is different from that of the NSJ. In particular it appears that the time-averaged momentum is more important than the unsteadiness in reducing the blockage with the use of the DSJ. More details on the flow process will be discussed further in subsection 4.2.5. 


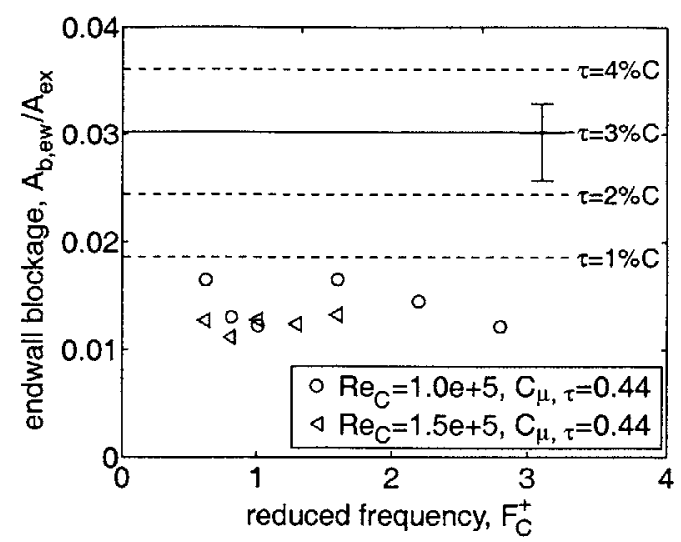

Figure 4.17 Frequency dependence of blockage reduction with DSJ over pressure surface of blade. $\tau=3 \% C$.

\subsubsection{Dependence on Pitchwise Location of DSJ}

Figure 4.18 shows endwall blockage vs. pitchwise location of the DSJ actuator. The actuator frequency and the amplitude are fixed at $F_{C}{ }^{+}=1.0$ and $C_{\mu, \tau}=0.88$. A substantial reduction in the endwall blockage is achieved when the DSJ is located near the pressure side of the blade $(y=-0.04$ pitch $)$ or the tip clearance vortex core.

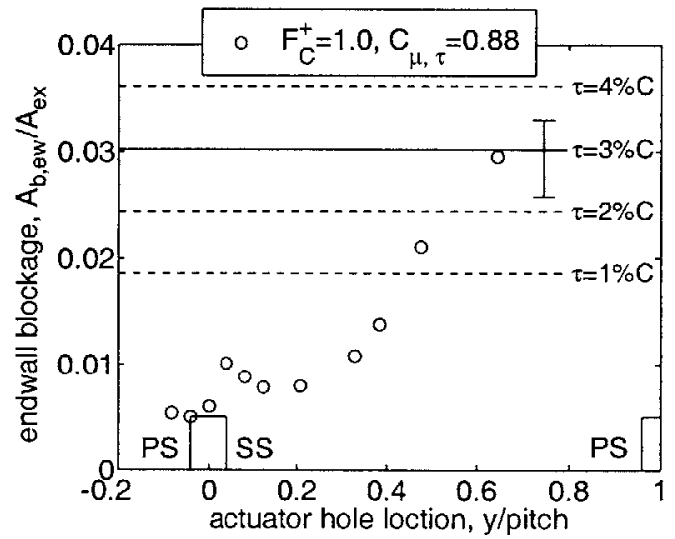

Figure 4.18 Endwall blockage vs. pitchwise location of DSJ actuator. $\tau=3 \% C$.

Figure 4.19 shows mass-averaged and stream thrust-averaged total pressure loss coefficients. Unlike NSJ, the DSJ reduces both of them significantly from the baseline when the DSJ is located from -0.08 pitch to about 0.6 pitch. 


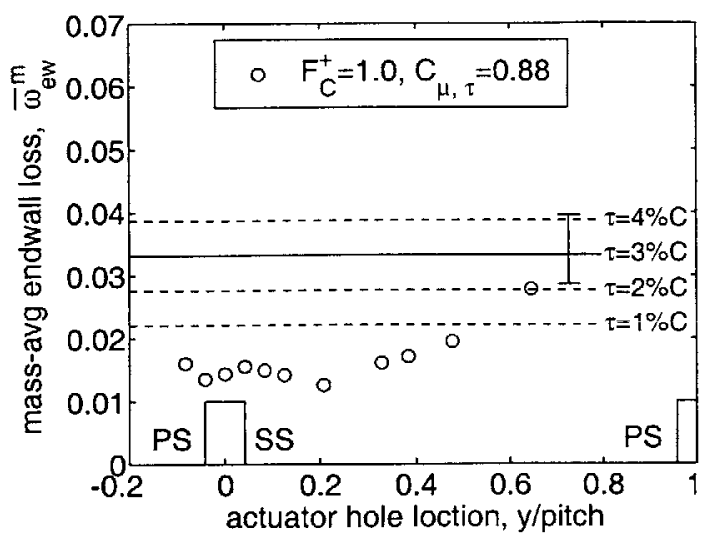

(a)

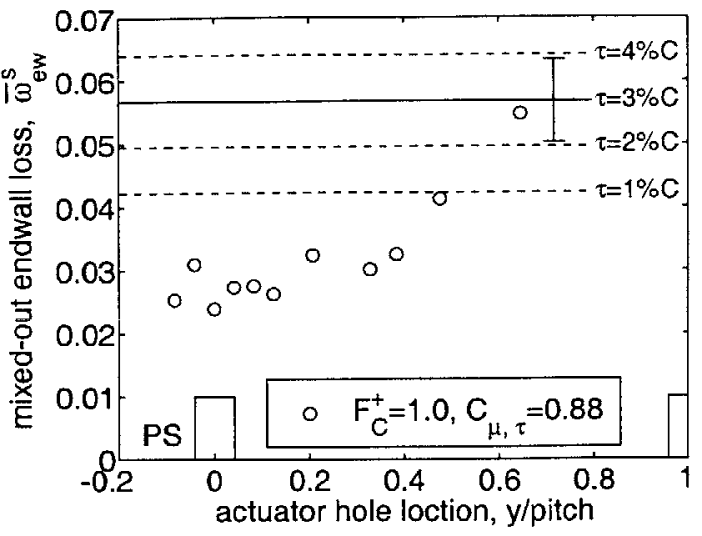

(b)

Figure 4.19 Endwall total pressure loss coefficient vs. pitchwise location of DSJ actuator $(\tau=3 \% C):($ a) mass-averaged $\omega$, (b) stream thrust-averaged (or fully mixed-out) $\omega$.

\subsubsection{Contribution of Each DS.J Actuator}

As with the use of NSJ, the endwall blockage has been measured with one DSJ actuator at a time to examine the contribution of each. Figure 4.20 shows endwall blockage plotted against the actuator amplitude for DSJ placed over the pressure side of the blade. None of the three DSJ actuators dominates and the blockage reduction is almost the same regardless of actuator chordwise location. Similar to the NSJ, three chordwise locations of the DSJ considered in this study are equally effective in blockage reduction.

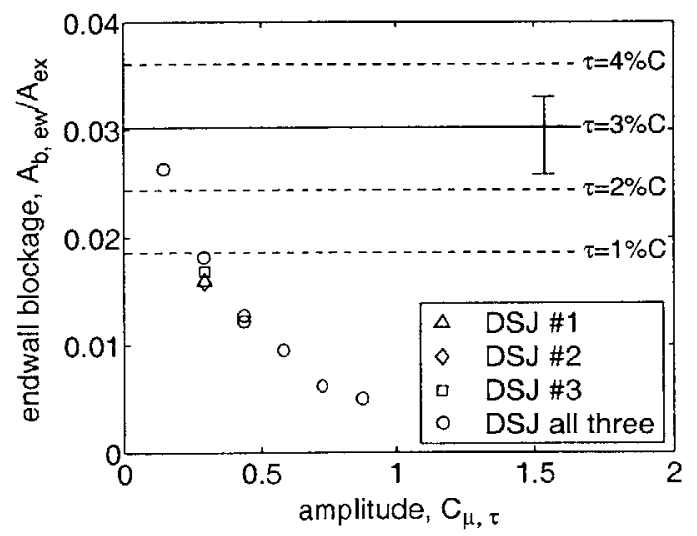

Figure 4.20 Contribution of each DSJ actuator placed over blade PS. Amplitude is total $C_{\mu, \tau}$ from all working actuators. $\tau=3 \% C$ and $F_{C}{ }^{+}=1.0$. 


\subsubsection{Comparison between DSJ and SDJ}

The measurements with the DSJ actuation presented above have revealed two aspects that support the hypothesis that momentum injection is mainly responsible for the observed blockage reduction with the use of DSJ. The first is that the reduction is not sensitive to the actuation frequency. The second is that the DSJ reduces both blockage and loss in the endwall region. SDJ (Steady Directed Jet) experiments have been carried out to examine the hypothesis by establishing the bulk behavior, time-averaged momentum of the directed jet and blockage reduction. Figure 4.21 shows the comparison between DSJ and SDJ. Note that $C_{\mu, \tau}$ of the DSJ has been time-averaged as shown in Figure 2.9(b). The data essentially collapse to a single curve verifying that the time-averaged $C_{\mu, \tau}$ is the important parameter in reducing the blockage with the use of directed jets.

Figure 4.21 shows that the tip clearance-related endwall blockage can be completely eliminated using directed jets with a time-averaged momentum flux that is about 13 to $19 \%$ of the momentum flux of the leakage flow. In other words, endwall blockage can be reduced to the level of the extrapolated baseline with zero clearance (See Figure 3.5) when directed jets are applied at a time-averaged momentum coefficient of roughly 0.16 .

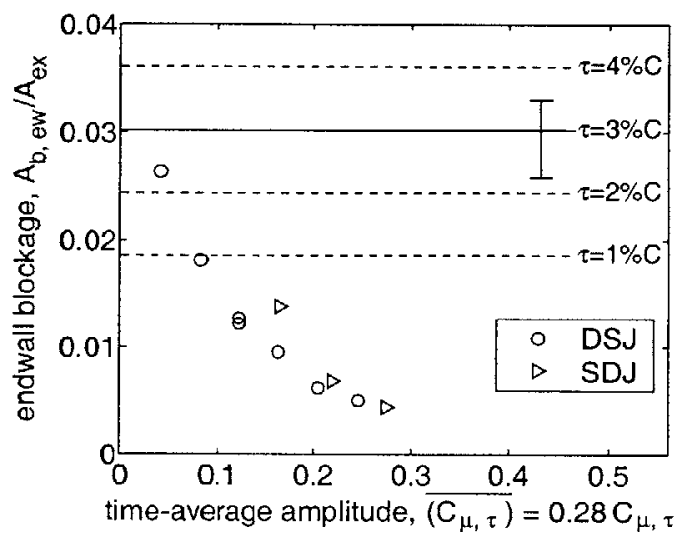

Figure 4.21 Comparison between DSJ and SDJ (Steady Directed Jet). Holes of both actuators placed over pressure surface of blade. $\tau=3 \% C$. DSJ at $F_{C}{ }^{+}=1.0$.

\subsubsection{Effect on Overall Efficiency}

As presented above, all three actuators (NSJ, DSJ, and SDJ) are capable of mitigating the endwall blockage associated with tip leakage flow but another important aspect of the tip leakage flow is the loss incurred. To determine whether the reduction in endwall loss with the use of directed jets results in an improvement of the overall 
efficiency, the reduction in the endwall flow power deficit must be compared with the flow power expended by the actuator. The endwall flow mechanical power deficit is:

$$
\Pi_{\text {deficit }}=\frac{\dot{m}_{i n}}{\rho}\left(P_{t, \infty}-\bar{P}_{t}^{m}\right)=\frac{\dot{m}_{i n} q_{\infty} \bar{\omega}_{e w}^{m}}{\rho}=Q_{i n} q_{\infty} \bar{\omega}_{e w}^{m}=U_{\infty} \cos \beta_{i n} \frac{h s}{2} q_{\infty} \bar{\omega}_{e w}^{m}
$$

The time-averaged flow power expended by the directed jets is:

$$
\Pi_{J}=\overline{\left(Q_{J} q_{J}\right)}=\frac{1}{2} \rho A_{J} \overline{U_{J}^{3}}=\frac{1}{2} \rho A_{J}\left(0.26 \times U_{J, p e a k}^{3}\right) .
$$

The factor of 0.26 in the above equation is from the time-averaging of the DSJ injection cycle (similar to that in Figure 2.9). The ratio of endwall loss reduction to expended flow power, $\eta_{J}$ is thus:

$$
\eta_{J}=\frac{\Delta \Pi_{\text {deficit }}}{\Pi_{J}} \times 100=\frac{\left(\Pi_{\text {deficit }}\right)_{\text {baseline }}-\left(\Pi_{\text {deficit }}\right)_{\text {control }}}{\Pi_{J}} \times 100(\%) .
$$

Figure 4.22 shows the values of $\eta_{J}$ for DSJ and SDJ at $\bar{C}_{\mu, \tau}=0.25$. Only about $16 \%$ of the flow power expended by the DSJ and about $30 \%$ of the flow power expended by the SDJ are regained in increasing the flow power measured $5 \% \mathrm{C}$ downstream of the trailing edge plane (or in reducing the mass-averaged total pressure loss) and the remainder is lost. Consequently, there is a net loss in the flow power with the use of directed jet actuation.

The difference in $\eta_{J}$ of DSJ and SDJ is because the flow power scales with the cube of the jet velocity. The cyclic DSJ has a peak jet velocity about twice the SDJ velocity for the same time-averaged momentum coefficient. The amount of the reduction in $\Pi_{\text {deficit }}$ is the same for both the DSJ and the SDJ for a given time-averaged momentum coefficient. Therefore, a higher $\Pi_{J}$ of DSJ for the same $\Delta \Pi_{\text {deficit }}$ results in a lower $\eta_{J}$ compared to SDJ.

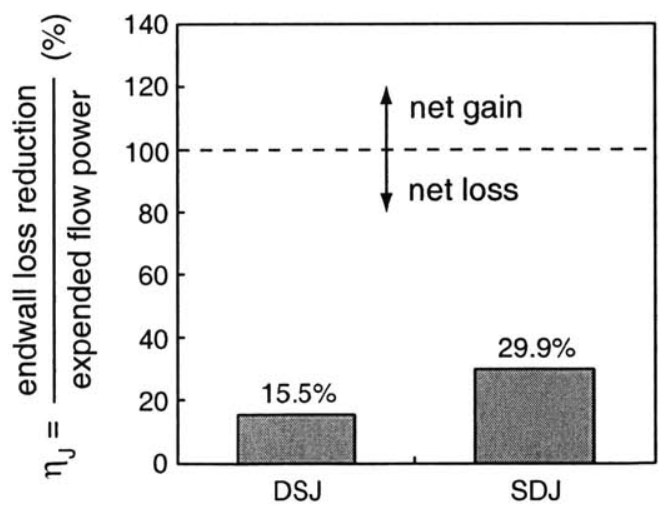

Figure 4.22 Ratio of endwall loss reduction to expended flow power of directed jet actuation at $\bar{C}_{\mu, \tau}=\mathbf{0 . 2 5}$. 
Even for SDJ, $\eta_{J}$ is very low, when compared to the boundary layer separation control wherein active control benefits from great leverage achieving about $1600 \%$ return on the input electrical power to the actuator (McCormick [4.7]). The ratio $\eta_{J}$ that is well below $100 \%$ in the current tip leakage flow control suggests that there is no leverage for loss reduction, which one can take advantage of, at least with the schemes considered in this study. Thus, achieving overall gain in efficiency appears to be hard, although the clearance-related blockage can be eliminated using the directed jet actuators.

There are two reasons for low $\eta_{J}$ with the present directed jet actuation: (i) relative angle between the directed jet and the main flow, and (ii) mixing process of the high velocity jet with the passage flow. We can examine how much loss is attributed to each. To examine effect of the relative flow angle, it is assumed that only the jet flow power associated with the velocity component parallel to the main flow is regained in increasing blade passage flow power. Recovered flow power of the jet decreases with relative angle as shown in Figure 4.23(a). The relative angle in spanwise direction is $25^{\circ}$ as illustrated in Figure 2.4 and corresponding recovery rate is $74 \%$ of the expended flow power. In other words, $26 \%$ of the jet flow power is lost due to the relative angle between the jet and the main flow.

Mixing between the actuator jet and the blade passage flow also incurs loss. $U_{J, \text { peak }}$ of DSJ is about $6.3 U_{\infty}$, and $U_{J}$ of SDJ is about $3.4 U_{\infty}$ at $\bar{C}_{\mu, \tau}=0.25$. Loss due to mixing between two streams with different magnitude of velocities can be estimated using a control volume analysis and the result is shown in Figure 4.23(b) where estimated recovery rate of the expended flow power is plotted against the velocity ratio between the jet and the blade passage flow. The recovery rate of the expended flow power decreases quickly from $100 \%$ as the velocity ratio increases and is $46 \%$ with the velocity ratio of 3.4 (for SDJ). The remaining $54 \%$ of the flow power is lost in the mixing process.

Thus, estimated overall recovery rate of the flow power $\eta_{J}$ considering losses due to relative angle and high jet velocity is $34 \%(=0.74 \times 0.46)$ for the SDJ explaining observed $\eta_{J}$ of $30 \%$ shown in Figure 4.22 . The mixing loss associated with high jet velocity is the dominant loss mechanism of the present directed jet actuators. To increase the efficiency of the actuation while reducing the blockage, the jet must be aligned with the main flow direction and the velocity ratio must be reduced close to unity. In particular, the $\eta_{J}$ of the present DSJ and SDJ can be improved by reducing the jet velocity while keeping the momentum coefficient the same. 


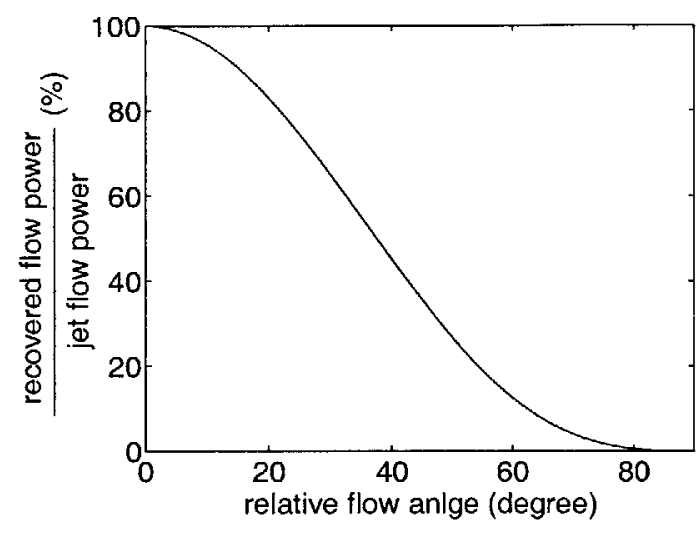

(a)

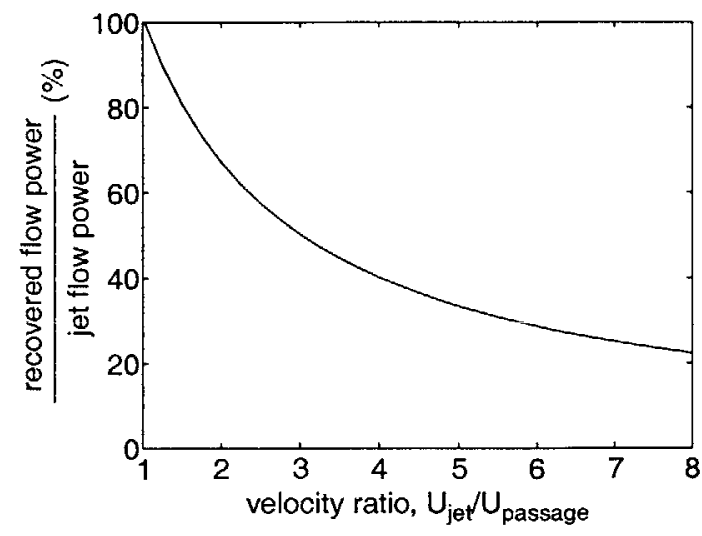

(b)

Figure 4.23 Estimated recovery rate of expended flow power: (a) as function of relative angle between jet and main flow; (b) as function of velocity ratio for fixed momentum injection.

\subsection{SUMMARY}

In this chapter, time-averaged effects of three types of actuators (namely NSJ, DSJ, and SDJ) on the tip clearancerelated blockage and loss have been discussed along with the bulk flow processes responsible for the observed changes in the blockage and loss. The key points made in this chapter are as follows:

- NSJ (Normal Synthetic Jets) at locations directly over the blade tip and tip clearance vortex core are effective in reducing the flow blockage associated with tip clearance flow when actuated at optimum reduced frequency.

The scaling for this frequency will be discussed in Chapter 5 .

- The effect of NSJ on mass-averaged endwall loss measured near the blade trailing edge plane is minimal with the actuation directly above the blade tip region and increases at other pitchwise locations with the largest increase the actuation is over the tip clearance vortex core.

- Based on analyses of blockage and loss measurements and the flow visualization, the reduction in blockage is associated with mixing enhancement and tip leakage flow reduction when the NSJ is over the blade tip, and with only mixing enhancement when the NSJ is over the tip clearance vortex core. 
- DSJ (Directed Synthetic Jets) are effective in reducing not only the tip clearance-related blockage but also the total pressure loss measured near the blade trailing edge plane. The result is much less insensitive to the frequency compared to the NSJ.

- For a given actuation amplitude (time-averaged momentum coefficient), DSJ and SDJ are about twice as effective as NSJ in reducing tip clearance-related blockage. It was demonstrated that the tip clearance-related endwall blockage could be eliminated using directed jets with time-averaged momentum flux that is roughly $16 \%$ of the momentum flux of the leakage flow.

- The reduction of blade exit plane blockage and loss by DSJ is associated with time-averaged streamwise momentum injection. This conclusion is based on the following observations: (1) the blockage reduction is not sensitive to the forcing frequency, (2) the use of SDJ (Steady Directed Jets) demonstrates the same amount of blockage reduction when the SDJ momentum coefficient and the DSJ time-averaged momentum coefficient are the same.

- Achieving overall gain in efficiency appears to be hard; the increase in flow power at blade exit is only about $30 \%$ of the expended flow power from the present SDJ actuator, which is the best among the actuators considered. To improve the efficiency of the directed jet actuation, both the direction and the magnitude of the jet velocity must be made close to the main flow velocity.

Although tip clcarance-related blockage reduction has been demonstrated based on the mixing enhancement and the leakage flow reduction schemes with the use of NSJ, the momentum flux produced with the NSJ actuation is not used as efficiently as in the case with the DSJ or SDJ. Therefore, the use of NSJ is not cost effective and it may not be worthy of any further pursuit. The streamwise momentum injection using the SDJ should be further explored for potential in improving compressor stability while minimizing penalty on efficiency. 


\section{REFERENCES}

[4.1] Rains, D. A., "Tip Clearance Flows in Axial Flow Compressors and Pumps", California Institute of Technology, Hydrodynamics and Mechanical Engineering Laboratories, Report No. 5, 1954.

[4.2] Storer, J. A. and Cumpsty, N. A., "Tip Leakage Flow in Axial Compressors", ASME Paper No. 90-GT-127, 1990.

[4.3] Katz, Y., Horev, E., and Wygnanski, I., "The Forced Turbulent Wall Jet", J. Fluid Mech., vol. 242, pp. 577$609,1992$.

[4.4] Tsuji, Y., Morikawa, Y., Nagatani, T., and Sakou, M., "The Stability of a Two-dimensional Wall Jet", Aeronautical Quarterly, XXVIII, pp. 235-246, November 1977.

[4.5] Kang, E., Breuer, K. S., and Tan, C. S., "Control of Leakage Flows Using Periodic Excitations", AIAA 20002232, 2000.

[4.6] Storer, J. A. and Cumpsty, N. A., "An Approximate Analysis and Prediction Method for Tip Clearance Loss in Axial Compressors", ASME Paper No. 93-GT-140.

[4.7] McCormick, D. C., "Boundary Layer Separation Control with Directed Synthetic Jets", AIAA 2000-0519. 


\section{CHAPTER 5 UNSTEADINESS IN TIP CLEARANCE VORTEX}

\subsection{INTRODUCTION}

As described in Chapter 4, an optimum frequency exists for blockage reduction with NSJ actuation. This optimum scales with upstream velocity, but not with tip clearance, and on a tentative basis, blade chord length was used as the length scale. The bulk flow process measurements identified the promotion of mixing between the low streamwise momentum flow in the vortex core and the high momentum main flow when the NSJ is operated at the optimum frequency. What has not been discussed is why mixing enhancement occurs at that frequency and what sets the frequency.

Increased mixing is a result of increased momentum transport/transfer, which can be attributed to higher levels of unsteadiness. In other words, it is suggested that the sensitivity of the blockage reduction to forcing frequency is because the tip clearance vortex is more receptive to NSJ actuation at certain frequency. If so the implication is that there is a natural frequency of the tip clearance vortex at which it is more receptive to NSJ actuation because of resonance. Time-resolved hot-wire measurements discussed in this chapter are analyzed to test this hypothesis. A simple model is used to address the issue of what sets this observed frequency.

\subsection{TIME-RESOLVED MEASUREMENTS}

\subsubsection{Natural Response}

To examine the existence of a dominant frequency in the tip clearance vortex, time-resolved measurements were taken using a hot-wire sensor placed $5 \% \mathrm{C}$ downstream of blade trailing edge plane. The RMS fluctuation level of the velocity trace measured near the vortex core is about $15 \%$ of time-averaged free-stream velocity, roughly two orders of magnitude larger than that measured near mid-span (about $0.2 \%$ ), even without actuation.

The frequency content of the velocity trace measured near the vortex core exhibits a peak, most identifiable in the data taken with the hot-wire sensor placed between the center of the vortex core and the casing wall. To examine how the peak frequency scales, the frequency contents of eight velocity traces taken with threc clearances, two 
upstream velocities, and two hot-wire orientations are plotted against different normalizations of measured frequency as shown in Figure 5.1(a) - (c). The two upstream velocities create two peaks when plotted against dimensional frequency (as shown in Figure 5.1(a)) indicating they should be scaled with upstream velocity. In Figure 5.1(b), the abscissa is the frequency parameter $\beta_{\tau}=2 \pi f \tau / U_{\infty}$. Using this, the peaks still do not coincide. Figure $5.1(\mathrm{c})$ has ${F_{C}}^{+}=f C / U_{\infty}$ as abscissa. With this choice of parameter the peaks do line up vertically at approximately $F_{C}{ }^{+}=0.75$ showing that there is a periodic unsteadiness in the tip clearance vortex at this frequency.
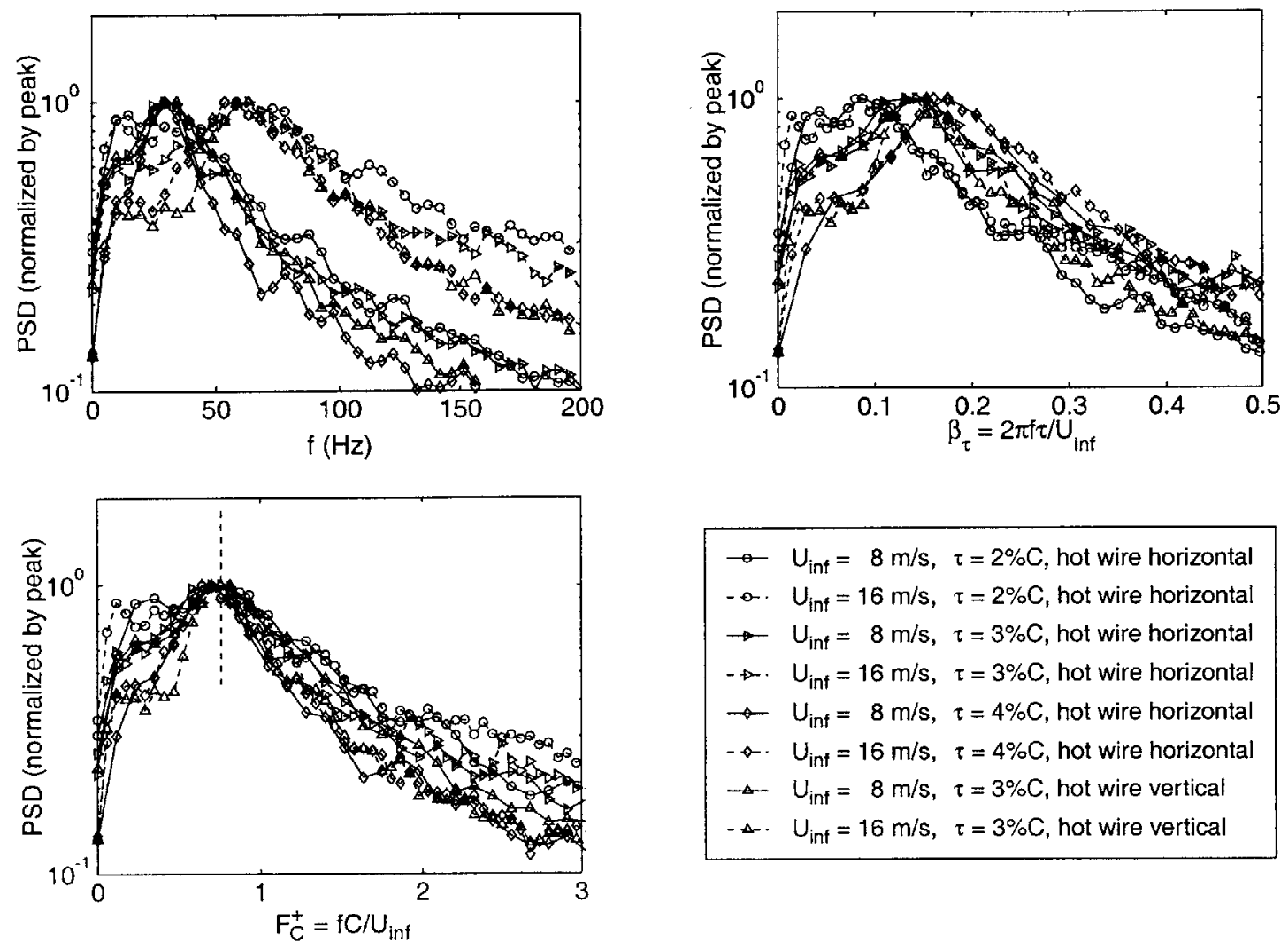

$$
\begin{aligned}
& \rightarrow U_{\text {inf }}=8 \mathrm{~m} / \mathrm{s}, \tau=2 \% \mathrm{C} \text {, hot wire horizontal } \\
& -0 . U_{\text {inf }}=16 \mathrm{~m} / \mathrm{s}, \tau=2 \% \mathrm{C} \text {, hot wire horizontal } \\
& \rightarrow \quad U_{\text {inf }}=8 \mathrm{~m} / \mathrm{s}, \tau=3 \% \mathrm{C} \text {, hot wire horizontal } \\
& \rightarrow \quad U_{\text {inf }}=16 \mathrm{~m} / \mathrm{s}, \tau=3 \% \mathrm{C} \text {, hot wire horizontal } \\
& \rightarrow-U_{\text {inf }}=8 \mathrm{~m} / \mathrm{s}, \tau=4 \% \mathrm{C} \text {, hot wire horizontal } \\
& -\diamond-U_{\text {inf }}=16 \mathrm{~m} / \mathrm{s}, \tau=4 \% \mathrm{C} \text {, hot wire horizontal } \\
& \rightarrow-U_{\text {inf }}=8 \mathrm{~m} / \mathrm{s}, \tau=3 \% \mathrm{C} \text {, hot wire vertical } \\
& -\Delta U_{\text {inf }}=16 \mathrm{~m} / \mathrm{s}, \tau=3 \% \mathrm{C} \text {, hot wire vertical }
\end{aligned}
$$

Figure 5.1 Frequency contents of velocity measured with hot-wire placed in blade exit plane near casing wall. Data taken without actuation.

A hot-wire sensor oriented horizontally is much more sensitive to variations in yaw angle than in pitch angle of the incoming flow and vicc versa for a hot-wire sensor oriented vertically. It is thus inferred that the periodic unsteadiness of the tip clearance vortex involves motion in both horizontal (or pitchwise) and vertical (or spanwise) directions, because measurements with both orientations show the peak at the same frequency. We will revisit motion of the tip clearance vortex in subsection 5.5.4. 


\subsubsection{Forced Response}

To examine the response of the tip clearance vortex to the NSJ actuation, hot-wire measurements have been taken $5 \% C$ downstream of the blade trailing edge plane with the NSJ directly over the blade tip. Figure 5.2 shows the responses in both the endwall region and the mid-span region at seven discrete forcing frequencies. The amplitude in the endwall region is more than two orders of magnitude higher than that in the mid-span region. Although the NSJ is directly over the blade tip, the effect of NSJ actuation is not limited to the tip clearance region and the tip clearance vortex in the blade passage is affected by the actuation. The response in the endwall region has a peak at around $F_{C}^{+}=0.75$ showing that the tip clearance vortex is more receptive at the same frequency that is most effective in reducing the endwall blockage with NSJ.

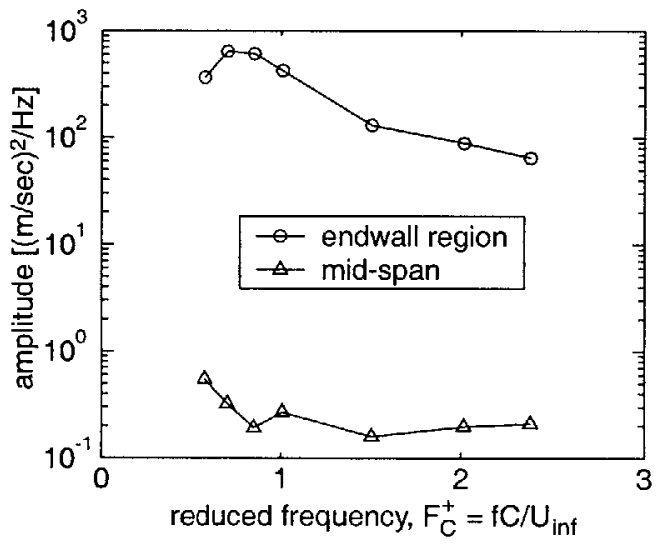

Figure 5.2 Forced response measured $5 \% C$ downstream of T.E. plane with NSJ over blade tip. $\tau=3 \% C$ and $C_{\mu \tau}=\mathbf{0 . 8 8}$.

\subsection{FREQUENCY OF TIP CLEARANCE VORTEX UNSTEADINESS}

Periodic unsteadiness in the tip clearance vortex has also been observed by others. Graf [5.1] (1996) first observed periodicity and motion of the tip clearance vortex in his study based on CFD simulations of the flow through a single stage (rotor followed by stator) compressor utilizing a three-dimensional time-accurate Reynolds-averaged Navier-Stokes solver. The period of the motion appeared to scale with the rotor flow-through time rather than with the stator-passing period. However, a physical mechanism that drives the periodic unsteadiness was not identified despite the attentions and discussions on this observation. 
More recent work by Mailach et al. [5.2] (2001) provides time-resolved data taken in a low-speed compressor cascade with various tip clearances. They used microphones measuring pressure fluctuations at the casing about $20 \% C$ downstream of the blade exit plane and observed a peak in the frequency content similar to those in Figure 5.1 .

The data from the current cascade, Graf [5.1], and Mailach et al. [5.2] are shown in Figure 5.3. Flow conditions of these data points are shown in Table 5.1. Although the dimensional frequencies are different by an order of magnitude between that of the current cascade and that of Mailach et al. [5.2], the periodicities of all three data sets are about the same in terms of the reduced frequency $F_{C}{ }^{+}$and are approximately $F_{C}{ }^{+}=0.6 \sim 0.9$. In sections 5.4 and 5.5, we will present a plausible physical mechanism responsible for this periodic unsteadiness of the tip clearance vortex.

Table 5.1 Flow conditions of tip clearance vortex unsteady data.

\begin{tabular}{|c|l|l|l|l|l|}
\hline Symbol & Data & $R e_{C}$ & $\tau / C(\%)$ & Stagger angle $\left(^{\circ}\right)$ & Inlet flow angle $\left(^{\circ}\right)$ \\
\hline$\circ$ & Current cascade & $1.0 \times 10^{5}$ & 3.0 & 56.9 & 62.7 \\
\hline 0 & Current cascade & $2.0 \times 10^{5}$ & 3.0 & 56.9 & 62.7 \\
\hline$\square$ & Mailach et al. $[5.2](2001)$ & $4.0 \times 10^{5}$ & 4.3 & 49.5 & 62.0 \\
\hline$\square$ & Mailach et al. $[5.2](2001)$ & $4.0 \times 10^{5}$ & 3.0 & 49.5 & 62.0 \\
\hline$\triangleleft$ & Graf [5.1] (1996) & $10.7 \times 10^{5}$ & 1.6 & 57.5 (mid-span) & 62.9 (mid-span) \\
\hline$\triangleleft$ & Graf [5.1] (1996) & $10.7 \times 10^{5}$ & 1.6 & 57.5 (mid-span) & 64.3 (mid-span) \\
\hline
\end{tabular}

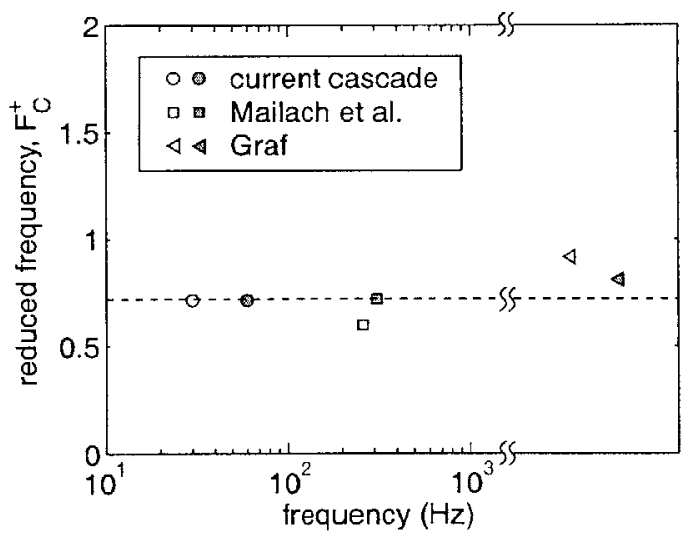

Figure 5.3 Reduced frequency of periodic unsteadiness of tip clearance vortex. Open and solid symbols denote different flow conditions as summarized in Table 5.1. 


\subsection{VORTEX INSTABILITY}

To attempt to explain the source of the unsteadiness in the tip leakage vortex, we examine the topic of Crow instability [5.3] associated with trailing vortices of wings. As shown in Figure 5.4, trailing vortices can exhibit a symmetric and nearly sinusoidal motion.

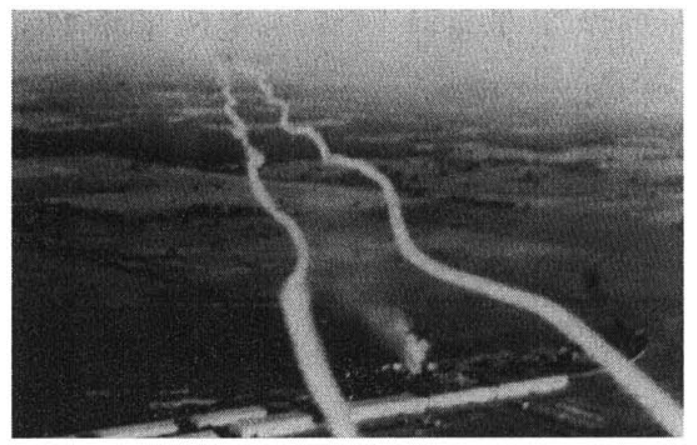

Figure 5.4 Instability of trailing vortices visualized by smoke [5.4]. View from chase airplane.

A theory by Crow [5.3] accounts for the instability during the early stages of the growth and predicts the most unstable wavelength for given vortex core radius, $R_{c}$ and separation distance, $b$ between two parallel counter-rotating line vortices (See Figure 5.5). Assuming solutions of exponential form $\vec{r}(x, t)=\hat{r} \times e^{a t+i k x}$ for a given wave number $k$, one can calculate a growth rate $a$ with the wave number corresponding to the maximum growth rate the most rapidly growing mode. The wavelength $\lambda=2 \pi / k$ depends only on the radius of vortex core, $R_{c}$ and the separation, $b$. Details on the calculation procedure of dispersion relations and consequent wavelength can be found in Crow [5.3].

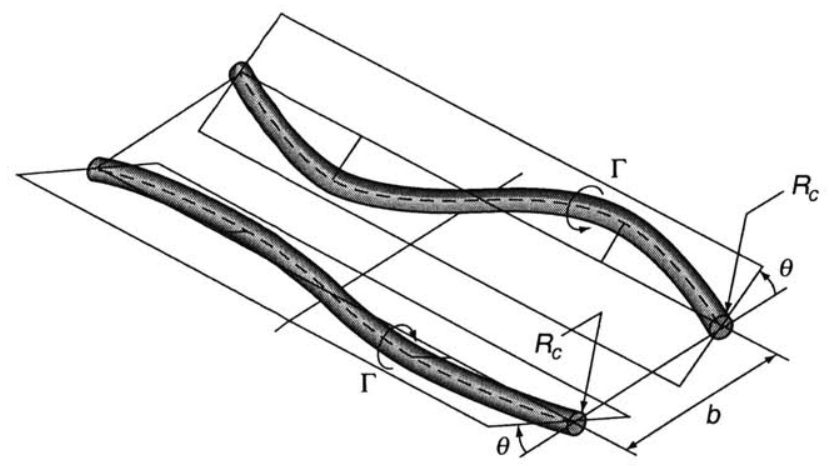

Figure 5.5 Schematic of trailing vortex instability.

Chevalier [5.4] carried out a series of flight tests with tip vortices visualized by smoke generated near wing tips as shown in Figure 5.4. One of his tests was a forced response test of the wing tip vortices by putting the airplane in a pitching motion at a fixed frequency. The optimum frequency to dissipate the trailing vortices was estimated by 
dividing the flight speed by the most unstable wavelength predicted by Crow's theory. The measured time for the trailing vortices to dissipate is plotted against the forcing frequency in Figure 5.6. The dissipation time is reduced by $50 \%$ when forced at the calculated optimum frequency. This experiment shows that mixing of the wing tip trailing vortices can be enhanced substantially by forcing them at the optimum frequency.

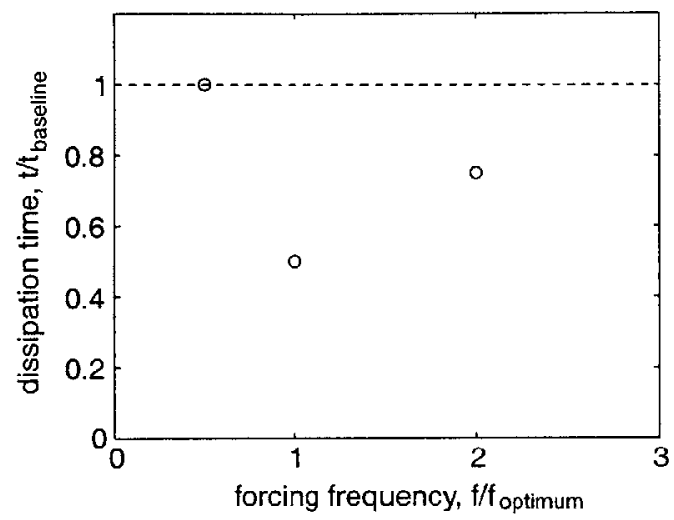

Figure 5.6 Dissipation time vs. forcing frequency of trailing vortices in flight test (data from Chevalier [5.4]).

\subsection{APPLICATION TO TIP CLEARANCE VORTEX}

\subsubsection{Analogy to Trailing Vortices}

As shown in Figure 5.7, the casing wall creates a mirror image of the tip clearance vortex resulting in a configuration similar to trailing vortices. Once the radius of vortex core $R_{c}$ and the separation distance $b$ are estimated, the Crow's instability analysis [5.3] can be applied to this counter-rotating vortex pair to calculate the most rapidly growing wavelength.

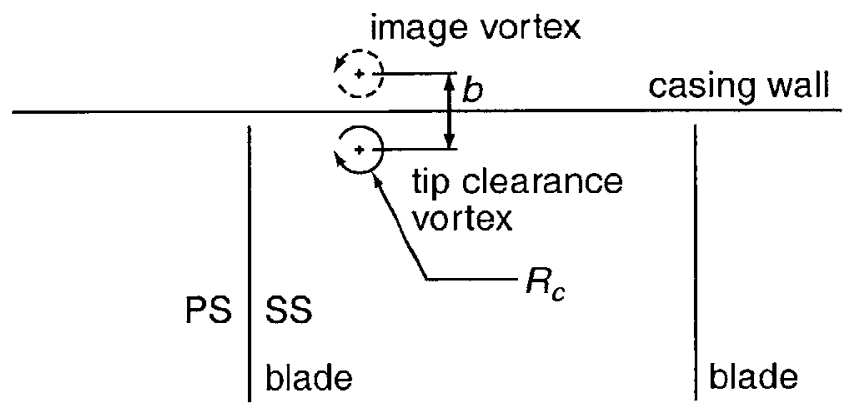

Figure 5.7 Schematic of tip clearance vortex and its image against casing wall creating a pair of counterrotating vortices similar to trailing vortices. 
There also exist other image vortices due to solid surfaces other than the casing wall. However, only the image associated with the casing wall is considered since the separation distance of other image vortices are larger except near the leading edge. As shown later, the instability or the peak in the frequency content does not develop until the vortex reaches near the blade exit plane and the effect of the image associated with the blade surface is thus assumed to be less important than the one associated with the casing wall.

\subsubsection{Vortex Core Radius and Separation Distance}

To apply Crow's theory to the tip clearance vortex system, we need to estimate the radius of vortex core $R_{c}$ and the separation distance $b$. The center of the tip clearance vortex is assumed to be located where the maximum total pressure loss coefficient is, which is marked as + in Figure 5.8. Based on CFD simulations, Crook [5.5] pointed out that the low total pressure center locates the vortex center with an error that is considerably smaller than the distance between the vortex center and the casing wall even with a significant radial non-uniformity in the incoming total pressure profile. The separation distance, $b$ is twice the distance between the center of vortex and the casing wall.

The radius of the vortex core $R_{c}$ is estimated using a formula given by Rains [5.6], who modeled the roll-up process of the tip clearance flow from the point of view of a two-dimensional unsteady flow. Assuming inviscid flow and a constant pressure difference along the chord, he computed the motion of the discontinuity surface or the sheet of vorticity as a function of non-dimensional time, $t^{*}$ :

$$
t^{*}=\frac{\chi}{\tau} \sqrt{\left(\Delta C_{P}\right)}
$$

where $\chi$ is the streamwise distance and $\overline{\left(\Delta C_{P}\right)}$ is the chordwise average loading. The non-dimensional radius of the vortex core is a function of $t^{*}$ only, i.e. $R_{c}{ }^{*}=f\left(t^{*}\right)$. Numerical calculations showed that $f\left(t^{*}\right)$ is well approximated by $0.14\left(t^{*}\right)^{0.85}$ or

$$
\frac{R_{c}}{\tau}=0.14 \times\left(\frac{\chi}{\tau} \sqrt{\overline{\left(\Delta C_{P}\right)}}\right)^{0.85}
$$

Thus the radius of the vortex core becomes

$$
R_{c}=0.14 \times \chi^{0.85} \tau^{0.15}{\overline{\left(\Delta C_{P}\right)}}^{0.425}
$$

To calculate $R_{c}, \chi$ is approximated as distance along the chord and $\overline{\left(\Delta C_{P}\right)}$ is estimated near mid-span. Axial views of the resulting vortex core radii for three clearances are shown in Figure 5.8(a) - (c). The elongation in pitchwise 
direction is due to the relative angle between the vortex trajectory and the total pressure survey plane, which is assumed to be the same as the exit flow angle, $51.8^{\circ}$. The view angle does not skew the vortex core in spanwise direction as illustrated in Figure 5.8(b).

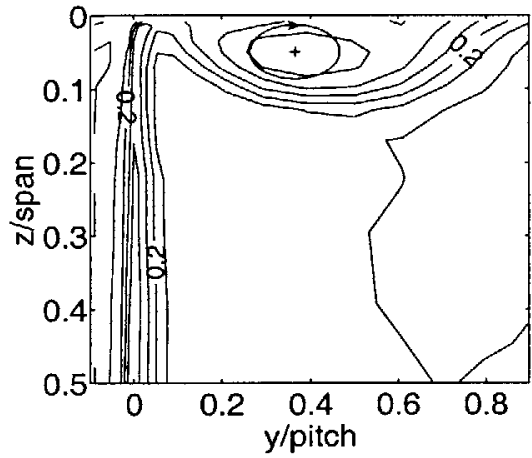

(a) $\tau=2 \% C$

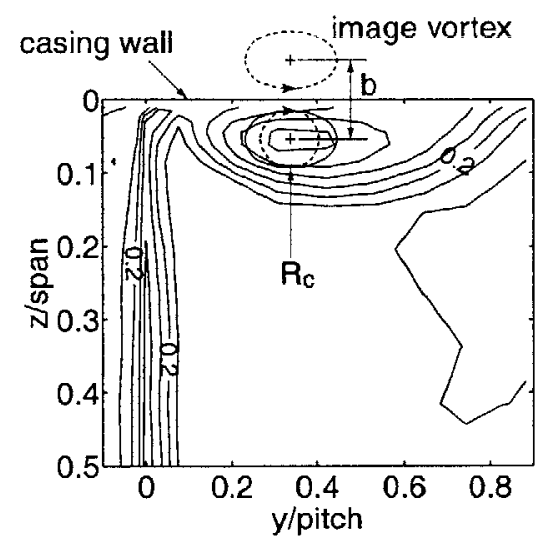

(b) $\tau=3 \% C$

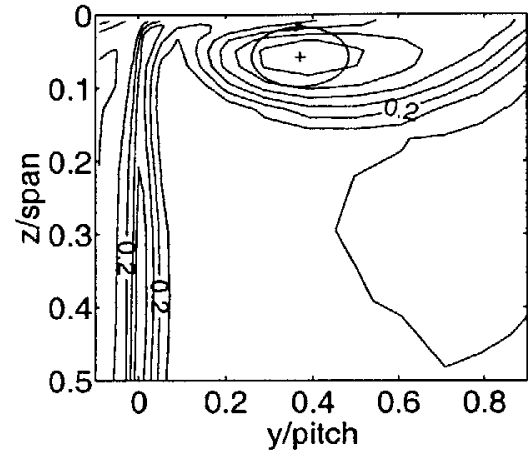

(c) $\tau=4 \% C$

Figure 5.8 Tip clearance vortex core estimated using Rains [5.6] model and location of maximum loss coefficient. Total pressure loss coefficient contour measured near trailing edge plane.

\subsubsection{Results of Vortex Instability Theory}

The Crow instability analysis can be applied to this counter-rotating vortex pair to calculate the most rapidly growing wavelength $\lambda$ using $R_{c}$ and $b$ as inputs to the calculation. A dispersion relation between non-dimensional wave number $\beta$ and amplification rate $\alpha$ is calculated following the procedure in Crow [5.3], and a result ${ }^{\S \S}$ for $\tau=$ $3 \% C$ is shown in Figure 5.9. The most rapidly growing wave number $\beta_{\max }$, which is a function of the ratio $R_{c} / b$ only, is determined as illustrated in Figure 5.9. The calculation is repeated for $R_{c} / b$ ranging from 0 to 0.5 and the resulting relation between the most unstable wave number $\beta_{\max }$ and $R_{c} / b$ is shown in Figure 5.10.

Corresponding to the symmetric long wave mode, which is the physical mode that actually occurs. See Crow [5.3]. 


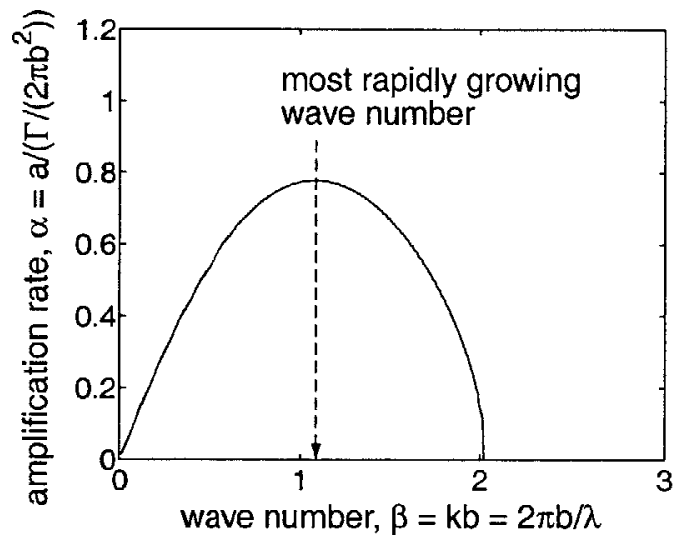

Figure 5.9 Amplification rate vs. wave number for $\tau=3 \% C . R_{c} / b=0.36$.

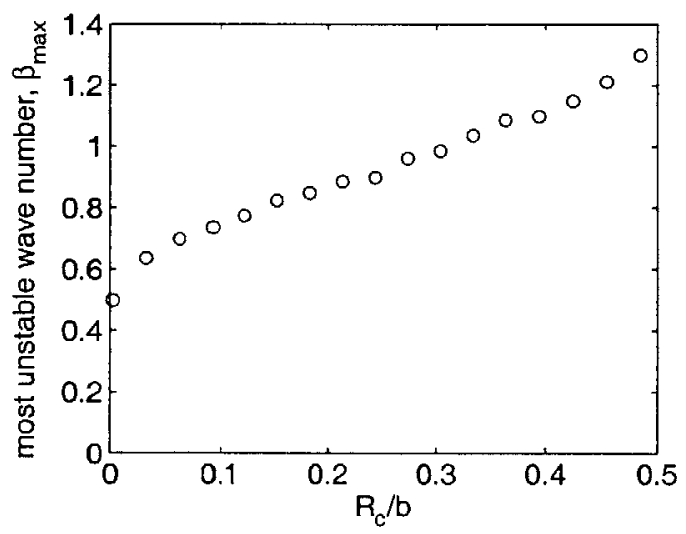

Figure 5.10 Most unstable wave number $\beta_{\max }$ calculated for various $\boldsymbol{R}_{c} / \boldsymbol{b}$.

Figure 5.11 shows estimated $R_{\mathrm{c}} / C$ and $b / C$ for three tip clearance sizes $(2 \%, 3 \%$, and $4 \% C)$. Contours in Figure 5.11 denote the most rapidly growing wavelength $\lambda / C$ for given $R_{c} / C$ and $b / C$. Variables are normalized by $C$, since the endwall flow structures scale with blade chord for a given clearance to chord ratio as pointed out by Storer [5.7]. The most unstable wavelength $\lambda$ is roughly the blade chord length $C$ (within $\pm 10 \%$ ) for three tip clearances. Once the most rapidly growing wavelength is given, the characteristic frequency can be estimated by assuming that the wavy pattern convects downstream at a speed equal to the magnitude of the far upstream flow velocity near midspan. Thus, the most unstable frequency is:

$$
f=U_{\infty} / \lambda
$$

The resulting characteristic frequencies for three clearances are compared with the measurements as shown in Figure 5.12. The calculated frequencies are close to the peaks of the measured power spectral density when plotted in the 
same dimensionless frequency axis, and are insensitive to the tip clearance size, e.g. $F_{C}{ }^{+}$decreases by only about $18 \%$ when $\tau / C$ increases by $100 \%$ (from $2 \% C$ to $4 \% C$ ).

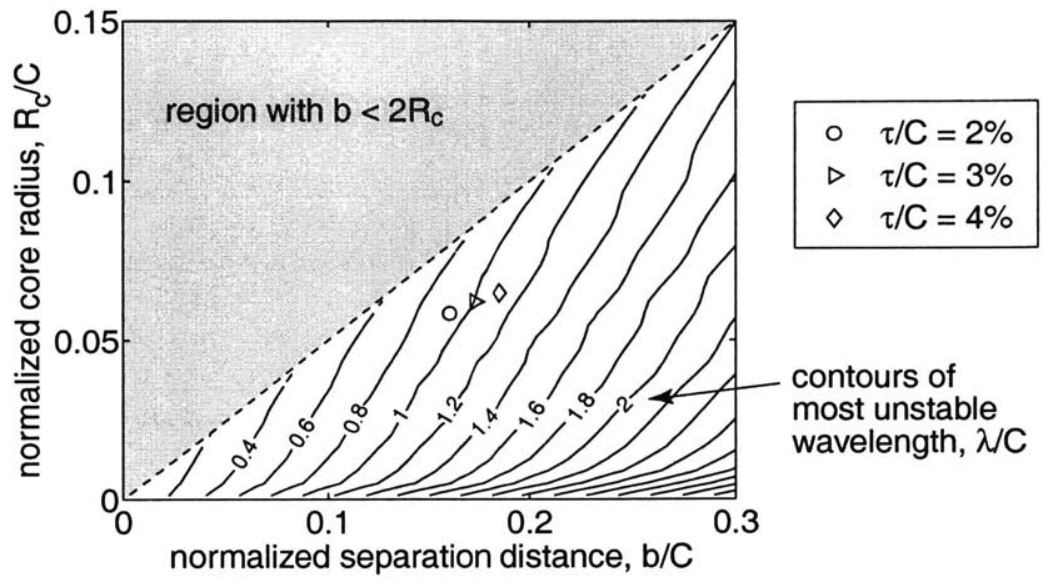

Figure 5.11 Contour plot of most rapidly growing wavelength $\lambda / C$ as function of vortex core radius and separation distance. Symbols are estimations for three tip clearance sizes as labeled.

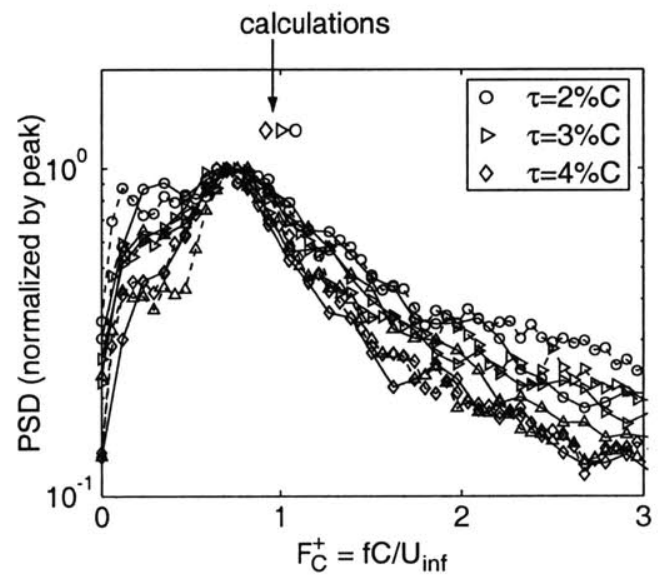

Figure 5.12 Comparison between measured frequency content and estimated frequency based on vortex instability.

\subsubsection{Relevance of Blade Chord: A Thought Experiment}

To examine the relevance of blade chord length as the length scale that sets the frequency of the vortex unsteadiness, a thought experiment is carried out. Figure 5.13 shows the tip clearance vortex and its image vortex for two blade chord lengths: (a) the current cascade with $C=0.190 \mathrm{~m}$ and (b) an imaginary compressor with $C=0.380 \mathrm{~m}$, i.e. with chord length twice that of the current cascade. Since the endwall structure scales with blade chord length for the 
same clearance to chord ratio as pointed out by Storer [5.7], the radius of the vortex core $R_{\mathrm{C}}$ and the separation distance $b$ would scale with $C$ as illustrated in the figure. Therefore, the wavelength $\lambda$ as well as the frequency $f$ scale with $C$.

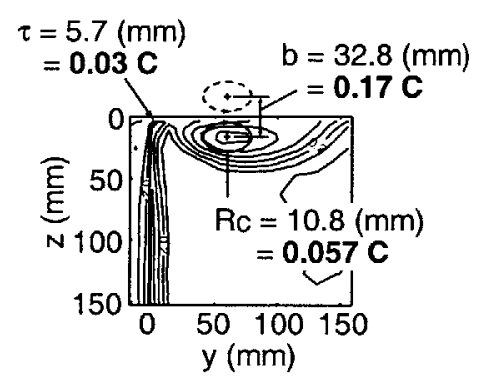

(a) $\mathrm{C}=190(\mathrm{~mm}), \tau=5.7(\mathrm{~mm})$

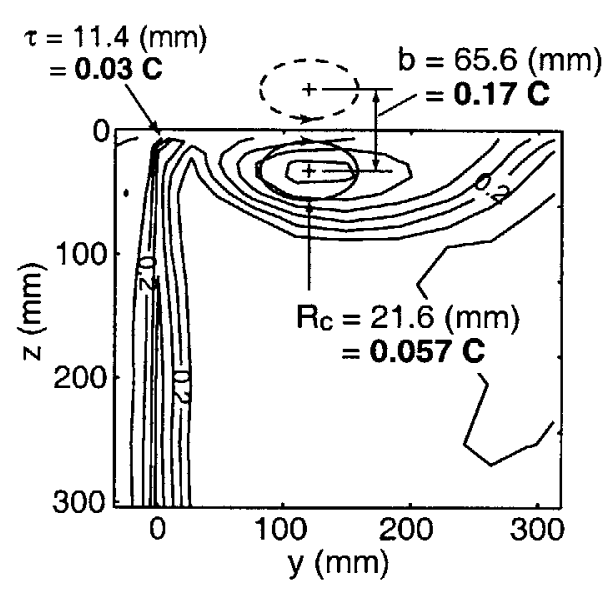

(b) $C=380(\mathrm{~mm}), \tau=11.4(\mathrm{~mm})$

Figure 5.13 Vortex core radius $R_{C}$ and separation distance $b$ for two blade chord lengths. Both with $3 \% C$ clearance.

Figure 5.14 shows the result of the stability calculation carried out for the above two geometries. The far upstream velocity is the same for both cases and is $10 \mathrm{~m} / \mathrm{sec}$. The dimensional frequency $f$ is $50 \%$ smaller for $380 \mathrm{~mm}$-chord. However, the frequency collapses to a single reduced frequency based on $C$ illustrating the relevance of the chord length.

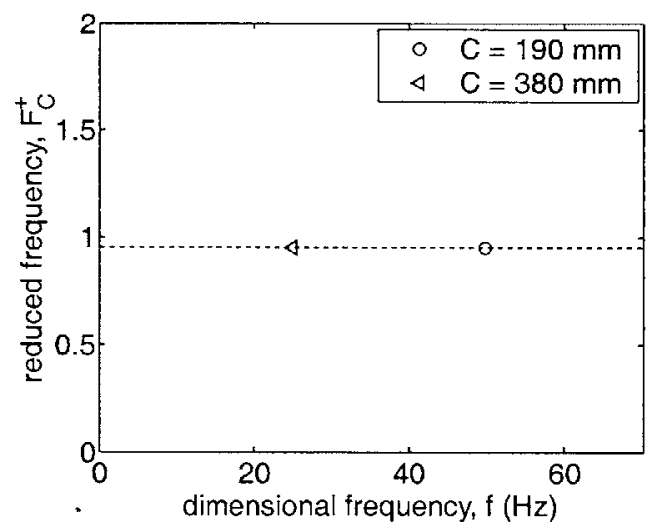

Figure 5.14 Result of thought experiment. Dimensional frequency vs. reduced frequency using blade chord length. 


\subsubsection{Motion of Tip Clearance Vortex}

The Crow's theory [5.3] predicts that the motion of the trailing vortices is confined in inclined planes with angle $\theta$ of roughly $45^{\circ}$ regardless of the wavelength (See Figure 5.5). Figure 5.4 seems to bear out this inclination. Thus, the motion of the tip clearance vortex should also be in a plane inclined by $45^{\circ}$. When viewed in axial direction, the amplitude of the motion in pitchwise direction is elongated due to the relative angle between the vortex and axial axes. Assuming that the relative angle is same as the exit flow angle $\left(51.8^{\circ}\right)$, the apparent angle of the inclined plane of the vortex motion is $58^{\circ}$ as illustrated in Figure 5.15. As described in subsection 5.2.1, the hot-wire measurements indicate that the periodic motion of the tip clearance vortex is in both pitchwise and spanwise directions, which is a necessary condition for a motion in an inclined plane. Zierke et al. [5.8] also observed unsteady wandering of tip clearance vortex in circumferential and radial directions using laser sheet visualization downstream of axial-flow water pump rotors.

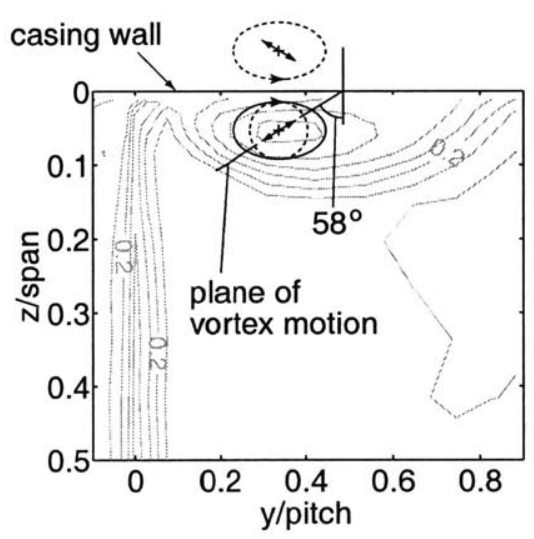

Figure 5.15 Axial view of vortex motion plane. Angle of plane estimated based on trailing vortex instability theory.

Growth rate of the instability, $\alpha$ is 0.79 from Figure 5.9. Time scale for the instability to grow $e^{1}=2.72$ times the initial amplitude of the perturbation is $1 / a$ and corresponding length scale is $U_{\infty} / a=U_{\infty} \times 2 \pi b^{2} /(\alpha \Gamma)$, which is roughly $1.3 C$ for the present cascade. In other words, amplitude of perturbation grows by a factor of 2.72 after convecting about $1.3 C$. Thus the amplitude of the vortex motion at the blade exit for the low-turbulence incoming flow of the current cascade (free-stream turbulence intensity $\sim 0.2 \%$ ) is suggested to be much smaller than the blade chord (or pitch). Time-averaged total pressure survey showing well-defined vortical structure within the blade passage (See Figure 5.15) also supports this scaling argument. 


\subsection{UNSTEADINESS AT VARIOUS AXIAL LOCATIONS}

In applying Crow's analysis in the axial plane near the trailing edge of the blades, we have made an approximation that the most unstable frequency is set by the local tip vortex core size and the local characteristics. The tip vortex core radius and the separation distance increase as one proceeds downstream while Crow's theory applies to the situation where the vortex core radius and the separation distance are invariant with downstream distance. The degree of the variations in the local characteristics along the streamwise location is addressed in this section.

The radius of the vortex core, $R_{c} / C$ (estimated using Rains' model) and the separation distance, $b / C$ (estimated assuming linear increase from $2 \tau / C$ to $b / C$ estimated in Figure 5.8(b)) are plotted against the distance along the chord, $\chi / C$ as shown in Figure 5.16(a). The rate of change, $\partial R_{c} / \partial \chi$ and $\partial b / \partial \chi$ is small (on the order of $10^{-1}$ ) and Crow's theory is applied on a local basis. The most unstable frequency estimated at each location, $\chi / C$ is shown in Figure 5.16(b). The calculated characteristic frequency decreases as one proceeds downstream, e.g. $F_{C}{ }^{+}$drops by about $30 \%$ over last $0.5 C$ (from $\chi=0.5 C$ to $C$ ).

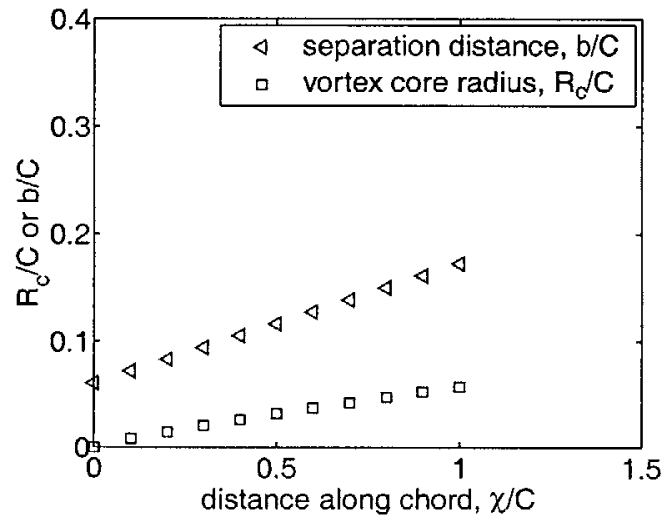

(a)

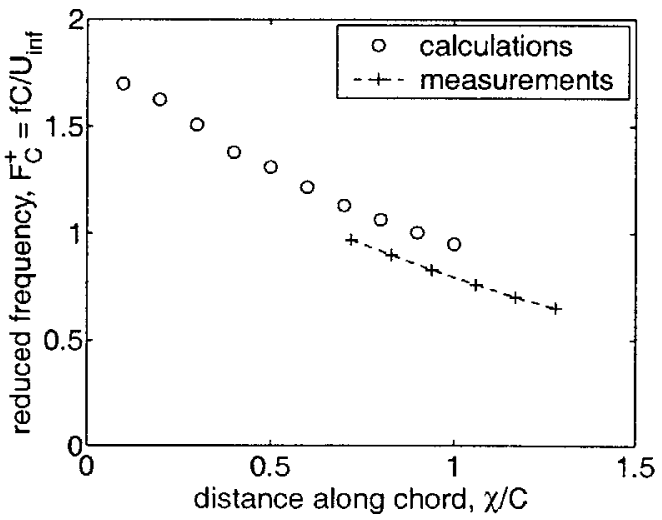

(b)

Figure 5.16 Variations along chord: (a) variations in $R_{c} / C$ and $b / C$ as function of $\chi / C$; (b) most unstable frequency $F_{C}{ }^{+}$calculated using local approximations.

Also plotted in Figure 5.16(b) are the peak frequencies in the measurements at various axial locations shown in Figure 5.17(a) using the hot-wire sensor, which is placed near the casing wall (approximately between the vortex core and the casing wall). The power spectral density at each location is shown in Figure 5.17(b). There is no dominant peak in the frequency content until about $\chi / C=0.94$. The peak grows downstream, which is conjectured to be an evidence of the development of the instability. Figure 5.17 (b) shows that the reduced frequency of the peak 
drops as one proceeds downstream, whose rate of decrease matches that of the calculated results as shown in Figure 5.16(b). Thus, the model applied on a local basis seems to bear out the trend in the measurements.

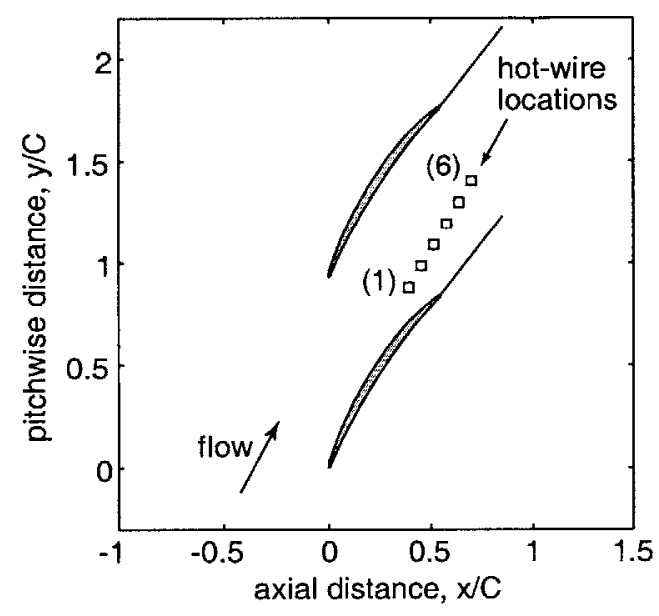

(a)

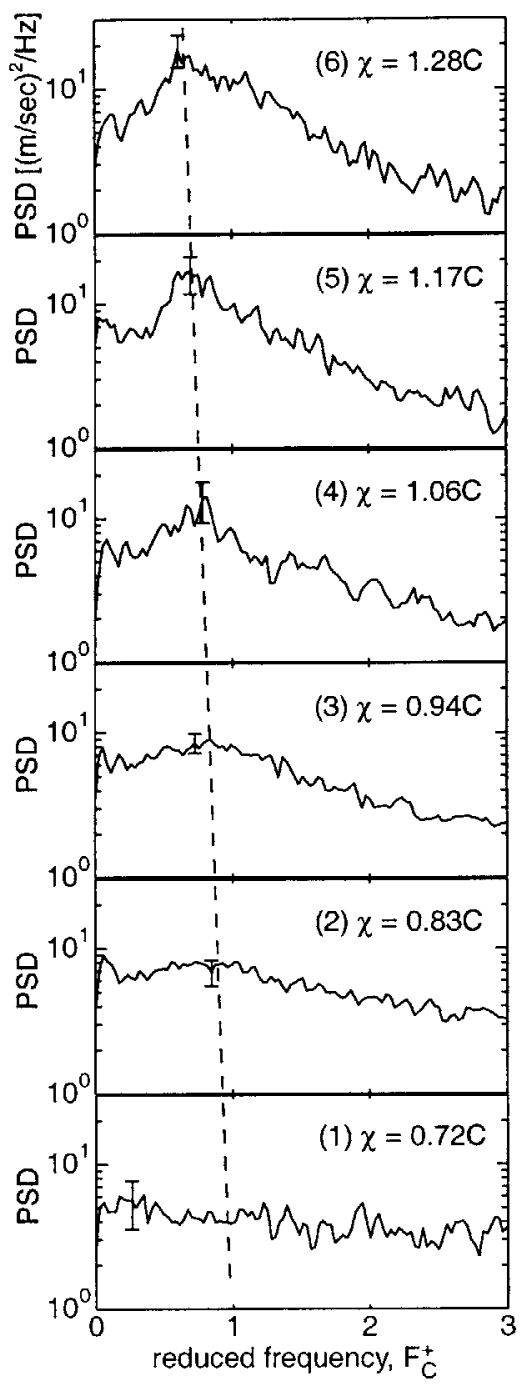

(b)

Figure 5.17 Time-resolved measurements at various axial locations: (a) locations of hot-wire sensor; (b) power spectral density at each location.

\subsection{SUMMARY}

In this chapter, the results of measurements of tip clearance vortex unsteadiness have been presented. The data show there exists a peak in the frequency content, i.e. a periodic unsteadiness, which corresponds to the most effective frequency for blockage reduction using the NSJ. It is suggested that the periodic unsteadiness of the tip clearance 
vortex can be related to the Crow instability [5.3], which predicts the most unstable wavelength of a counter-rotating vortex pair for given radius of vortex core and separation distance. Assuming that the phase velocity of the instability is equal to far upstream flow velocity near mid-span, the most unstable frequency of the vortex pair, which consists of the tip clearance vortex and its image associated with the casing wall, can be estimated.

Application of the theory to the current cascade and observations in other compressor geometries show that the time scale of the periodic unsteadiness and thus of the vortex instability is of the order of the convective time through the blade passage. Furthermore, frequency contents of the measurements at various axial locations show a growing peak, which is conjectured to be an evidence of developing instability.

Therefore, it is hypothesized that the frequency dependence of the blockage reduction with the use of NSJ actuation is because of the natural mode of instability associated with tip clearance flow configuration. When forced by the NSJ actuator near the natural frequency, the mixing between the tip clearance vortex and the main flow is promoted resulting in the observed endwall blockage reduction.

\section{REFERENCES}

[5.1] Graf, M. B., "Effects of Stator Pressure Field on Upstream Rotor Performance", Ph.D. thesis, MIT, June 1996.

[5.2] Mailach, R., Sauer, H., and Vogeler, K., "The Periodic Interaction of the Tip Clearance Flow in the Blade Rows of Axial Compressors", to appear in Proceedings of ASME Turbo Expo, New Orleans, June 2001.

[5.3] Crow, S. C., "Stability Theory for a Pair of Trailing Vortices", AIAA Paper No. 70-53, January 1970.

[5.4] Chevalier, H., "Flight Test Studies of the Formation and Dissipation of Trailing Vortices", J. Aircraft, Vol. 10, No. 1, January 1973.

[5.5] Crook, A. J., "Numerical Investigation of Endwall/Casing Treatment Flow Phenomena", GTL Report \#200, MIT Gas Turbine Laboratory, December 1989.

[5.6] Rains, D. A., "Tip Clearance Flows in Axial Flow Compressors and Pumps", California Institute of Technology, Hydrodynamics and Mechanical Engineering Laboratories, Report No. 5. 1954.

[5.7] Storer, J. A., "Tip Clearance Flow in Axial Compressors", Ph.D. Dissertation, University of Cambridge, January 1991.

[5.8] Zierke, W. C., Farrell, K. J., and Straka, W. A., "Measurements of the Tip Clearance Flow for a HighReynolds-Number Axial-Flow Rotor", ASME Journal of Turbomachinery, Vol. 117, pp. 522-532, October 1995. 


\section{CHAPTER 6 SUMMARY, CONCLUSIONS, AND}

\section{RECOMMENDATIONS}

\subsection{SUMMARY AND CONCLUSIONS}

Control of compressor tip clearance flows has been explored in a linear cascade using three types of fluidic actuators; Normal Synthetic Jet (NSJ; injection normal to the mean flow), Directed Synthetic Jet (DSJ; injection roughly aligned with the mean flow), and Steady Directed Jet (SDJ), mounted on the casing wall. The objective was to affect the following measures: (1) reduction of tip leakage flow rate, (2) mixing enhancement between tip leakage and core flow, and (3) increase in streamwise momentum of the flow in the endwall region. The results are summarized in the following:

(1) The NSJ provides mixing enhancement only, or both mixing enhancement and leakage flow reduction, depending on its pitchwise location. The DSJ and SDJ actuators provide streamwise momentum enhancement. Consequently, all three actuators result in a reduction of clearance-related blockage.

(2) For a given actuation amplitude, DSJ and SDJ are about twice as effective as NSJ in reducing clearance-related blockage. Further the DSJ and SDJ can eliminate clearance-related blockage with a time-averaged momentum flux roughly $16 \%$ of the momentum flux of the leakage flow.

(3) Effective pitchwise locations of actuator jets for blockage reduction are near the blade tip or the clearance vortex core.

(4) Achieving overall gain in efficiency appears to be hard; the decrease in loss is only about $30 \%$ of the expended flow power from the present SDJ actuator, which is the best among the actuators considered. To improve the efficiency of the directed jet actuation, both the direction and the magnitude of the jet velocity must be made close to the main flow velocity.

(5) Time-resolved measurements showed a periodic unsteadiness of tip clearance vortex, whose frequency corresponds to the most effective frequency for blockage reduction using the NSJ. Comparison between 
measurements and calculations based on trailing vortex instability theory implies that the observed periodic unsteadiness can be related to the vortex instability phenomenon.

(6) Application of the theory to the current cascade and observations in other compressor geometries show that the time scale of the periodic unsteadiness and thus of the vortex instability is of the order of the convective time through the blade passage.

The great leverage of the oscillatory blowing in boundary layer separation control could not be resembled in tip clearance flow control using the NSJ. In tip clearance flow control, the mixing enhancement only affects the diffusion of the velocity defect after it is formed as a result of the interaction between the leakage jet and the main flow. In contrast, unsteady forcing in boundary layer separation control prevents separation $a$ priori. Thus the inability of the mixing enhancement to prevent the loss generation mechanism a priori in tip clearance flow application is conjectured to be the reason for the inefficiency compared to the boundary layer separation control.

The results of the present work suggest the following in designing a scheme to effectively manage the tip clearance flow in axial compressors:

- Actuations based on the mixing enhancement and/or leakage flow reduction are not as effective as the one based on the streamwise momentum injection. Therefore, the use of NSJ (Normal Synthetic Jet) is not cost effective and is not worthy of any further pursuit.

- Steady directed jet (SDJ) is effective in reducing tip clearance-related blockage at design and should be further explored for potential on compressor stability.

- Instability of tip clearance vortex may be exploited by matching unsteadiness imposed by stators and the vortex instability frequency. 


\subsection{RECOMMENDATIONS FOR FUTURE WORK}

- Steady directed injection was found to the most effective means of managing the tip clearance flow among the actuations schemes considered in the present study and should be studied using steady numerical simulation.

- The hypothesis that the observed frequency in the tip clearance vortex is related to an instability of a vortex pair consisting of tip leakage vortex and its image associated with the casing wall can be examined by carrying out an experiment in which the radius of the tip clearance vortex core and the separation distance can be varied independently.

- Effect of matching unsteadiness imposed by stators and the vortex instability frequency should be studied using time-accurate numerical simulations. 


\section{APPENDIX A TWO-DIMENSIONAL LEAKAGE JET EXPERIMENT}

\section{A.1 INTRODUCTION}

At the outset of the current research, the initial idea behind the mixing enhancement scheme with the use of NSJ actuation was to take advantage of the shear layer instability (Drazin and Reid [A.1]). In other words, the original intension was to force the shear layer of the leakage jet at its resonance frequency to enhance mixing. A simple twodimensional model experiment was carried out to demonstrate the mixing enhancement using NSJ actuation and to measure the frequency response of the leakage jet in a more manageable environment compared to the tip leakage flow in compressor blade passages. Although it transpired, as presented in Chapter 5, that the roll-up of the tip clearance flow and subsequent formation of the vortex in the compressor blade passage bring about the mechanism (namely, vortex instability) that is different from the shear layer instability, the experimental setup and the results of the two-dimensional actuated wall jet experiment are documented in this appendix.

\section{A.2 EXPERIMENTAL SETUP}

Since the shear layer instability is a frequency-dependent phenomenon (Schober et al. [A.2], Michalke [A.3], and Fiedler et al. [A.4]), it is crucial to find the right frequency range. As shown in the above references, enhanced mixing increases spreading rate of shear layers, which is usually measured by the momentum thickness of the shear layer. Hence, the quantity of interest is the momentum thickness of the leakage jet at various forcing frequencies. A simple model experiment has been designed to measure the frequency response of a two-dimensional leakage jet.

Figure A.1 shows the schematic of the actuated wall jet experiment. A two-dimensional tip clearance flow is generated without the complication of the axial core flow and chordwise variations. It allows one to quantify the effects of the actuation to the leakage jet by measuring quantities such as the mean velocity profile, the flow rate through the clearance, and the pressure difference across the blade. 


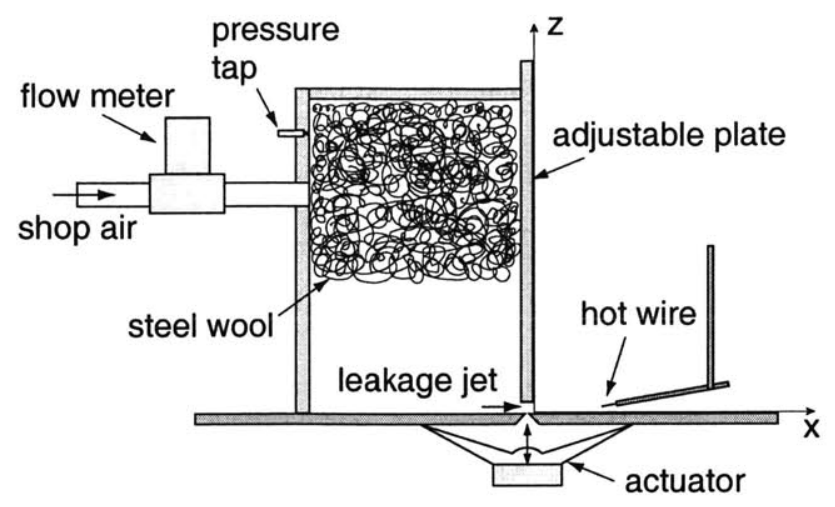

Figure A.1 Schematic of actuated wall jet experiment.

The experiment, which is similar to the one that was used by Kang et al. [A.5], consists of two major components - a pressurized box and a baseplate that accommodates the actuator. The pressurized box has an adjustable faceplate that can be adjusted up and down in the vertical direction so as to allow for variation in the size of the gap, which simulates the compressor tip clearance. The upper half of the pressurized box is filled with steel wool to dampen the inlet shop air and the unsteadiness that may exist with actuation. The leakage jet flows in $\mathrm{x}$ direction along the baseplate as indicated in Figure A.1 and is enclosed by two sidewalls that extend from each side of the pressurized box along the direction of the wall jet. The baseplate has a chamfered slit that can also be adjusted in width. A $30 \mathrm{~W} 20 \mathrm{~cm}$-diameter generic speaker is used in the actuated wall jet experiment where an actuator with a long span of slit is required to achieve two-dimensionality. The hot-wire sensor and traverse table described in subsection 2.2.2 are used to measure velocity profiles.

\section{A.3 HYDRODYNAMIC STABILITY CALCULATION}

The shear layer has inherent inviscid instability (Drazin and Reid [A.1]) and the most unstable frequency can be calculated for a given time-averaged velocity profile using hydrodynamic stability theory. To predict the most unstable frequency for the leakage flow, the hydrodynamic stability calculation has been carried out based on measured velocity profiles at three $x$ locations $(x / \tau=0,5$, and 9), which are shown in Figure A.2. To perform the stability calculation an analytical representation of the velocity profile suggested by Cohen as used in Katz et al. [A.6] is slightly modified as follows:

$$
U^{*}=A \tanh \left(\eta_{1}\right)+Q \frac{\ln \left(\eta_{2}\right)}{\eta_{2}} \quad \text { for } z^{*} \leq z_{m}^{*}
$$




$$
=\left[2\left(\frac{z^{*}}{z_{m}^{*}}\right)^{B}-\left(\frac{z^{*}}{z_{m}^{*}}\right)^{2 B}\right] \exp \left[-D\left(z^{*}-z_{m}{ }^{*}\right)^{C}\right] \quad \text { for } z^{*} \geq z_{m}{ }^{*}
$$

where $U^{*}=U / U_{m}, z^{*}=z / z_{m / 2}, \quad \eta_{1}=K z^{*} /\left(z_{m}{ }^{*}-z^{*}\right)^{0.5}$, and $\eta_{1}=1+(e-1) z^{*} / z_{m}{ }^{*}$. The six parameters; $A, Q, B, D, C$, and $K$ are then determined using a MATLAB ${ }^{\circledR}$ function "nlinfit", which performs a nonlinear least-squares data fitting to determine the coefficients of the nonlinear function. The velocity profiles represented by the fitted functions are shown in Figure A.2 with solid lines. A code written by Prof. Breuer was then used to perform the stability calculation, which solves the eigenvalue problem defined by the Orr-Sommerfeld equation (Drazin and Reid [A.1]) and the boundary conditions. Since the Orr-Sommerfeld equation accounts for viscosity, the code predicts two modes; inviscid mode associated with the shear layer and viscous mode associated with the wall boundary layer. Only the inviscid instability associated with the shear layer is considered here, as the intention is to enhance mixing between the ambient flow (or the main flow in casc of the compressor blade passage) and the leakage jet.
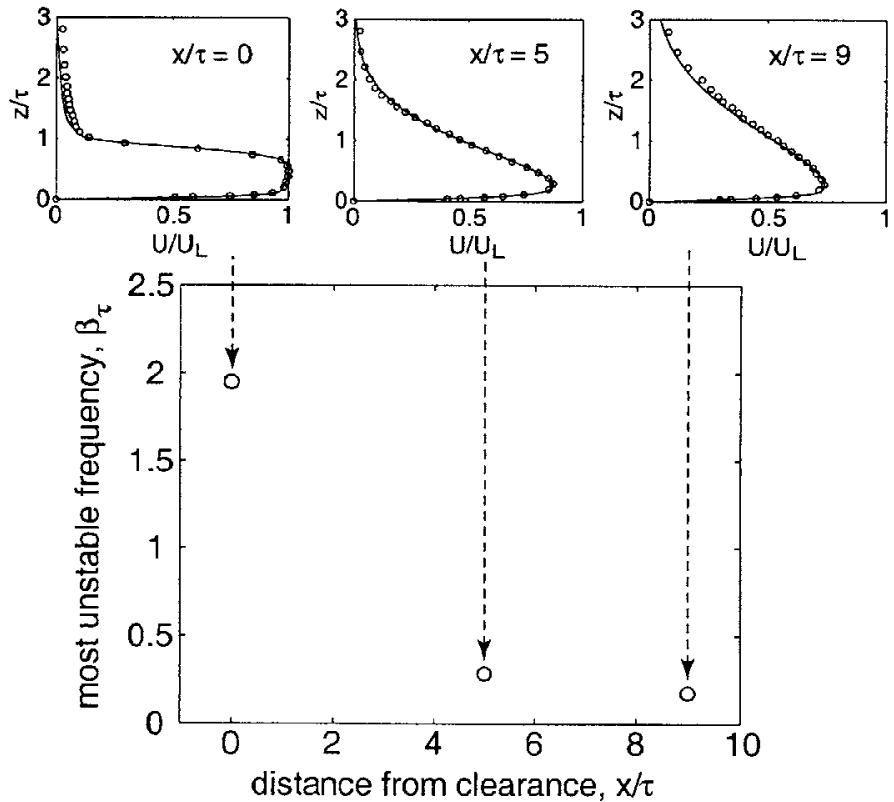

Figure A.2 Hydrodynamic stability calculation for velocity profiles of leakage jet at three downstream locations.

The calculated frequency for each velocity profile is plotted against the distance from the clearance gap in Figure A.2. The most unstable frequency drops quickly with the distance and is an order of magnitude smaller $\left(\beta_{\tau}=0.2\right)$ at $x / \tau=9$ than the initial frequency $\left(\beta_{\tau}=2\right)$ at $x / \tau=0$. 
What is of interest here is the forcing frequency that is expected to be most effective in enhancing the mixing of the shear layer. Observations reported in the literature (Oster and Wygnanski [A.7], and Schober [A.2]) is that the optimum frequency for mixing enhancement is about an order of magnitude lower than the initial instability frequency of the flow. Therefore, the best mixing enhancement is expected to occur at around the forcing frequency, $\beta_{\tau}=0.2$ for the wall jet considered here.

\section{A.4 EXPERIMENTAL RESULTS AND DISCUSSIONS}

The figure of merit for the effect of the actuation on the leakage jet is the mixing rate as reflected in the momentum thickness. As such, the momentum thickness of the shear layer is calculated from the time-averaged velocity profile measured five-clearance downstream of the clearance gap $(x=5 \tau)$ with a hot wire anemometer and is used as a measure of the mixing rate. The momentum thickness $\theta$ is non-dimensionalized by the clearance size $\tau$ as follows:

$$
\theta / \tau=f n_{1}\left(\beta_{\tau}, C_{\mu, \tau}\right)
$$

where the reduced frequency $\beta_{\tau}=2 \pi f \tau / U_{L}$ and the momentum ratio $C_{\mu, \tau}=\left(\rho U_{J, p e a k}^{2} d\right) /\left(\rho U_{L}^{2} \tau\right)$, which are the nondimensional frequency and amplitude of the actuation respectively.

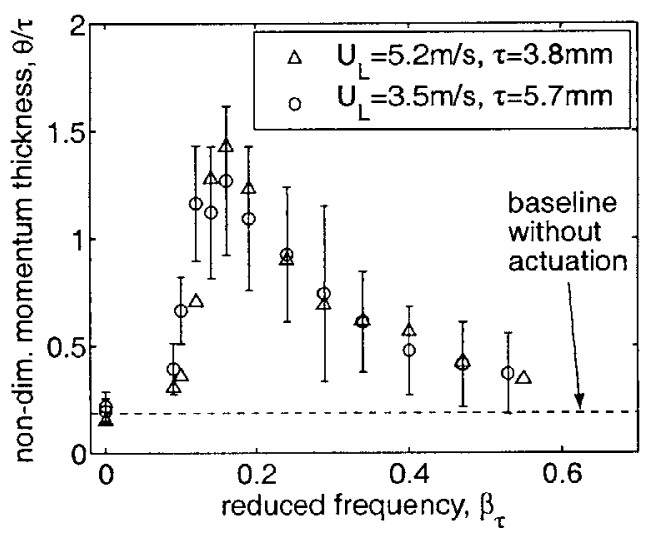

Figure A.3 Frequency dependence of mixing enhancement with actuation. Momentum thickness measured at $x=5 \tau$. Actuator slit aligned with pressure surface. Amplitude kept constant at $C_{\mu \tau}=1.0$. Actuator slit size, $d=1.0 \mathrm{~mm}$.

Figure A.3 shows the frequency-dependence of the momentum thickness of the shear layer. Two data sets measured with two different tip clearances collapse on to a single curve when non-dimensionalized. The actuator slit was aligned with the pressure surface of the faceplate. Note that the resonance frequency lies at $\beta_{\tau}=0.16$. 


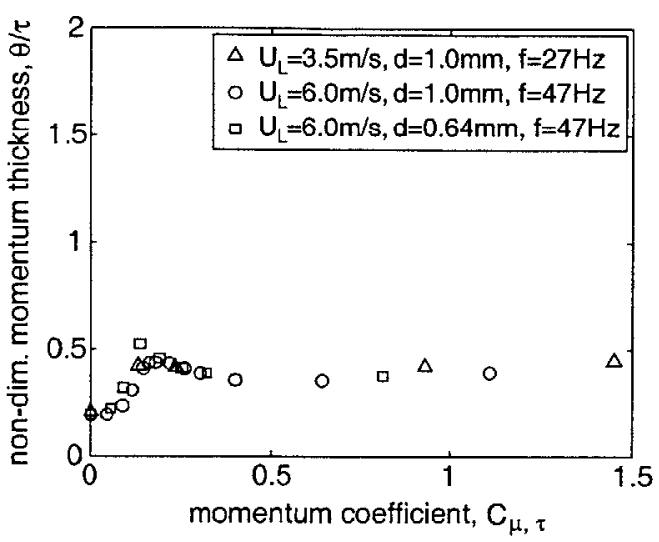

Figure A.4 Amplitude dependence of mixing with actuation. Momentum thickness of shear layer measured at $x=5 \tau$ with actuator slit aligned with suction surface. Non-dimensional forcing frequency, $\beta_{\tau}=0.28$. Tip clearance, $\tau=5.7 \mathrm{~mm}$.

Figure A.4 shows the amplitude-dependence of the momentum thickness. Three data sets with different leakage velocities and slit sizes are plotted in non-dimensional form. The non-dimensional forcing frequency $\beta_{\tau}$ is 0.28 , which is not the optimum for the mixing enhancement. The actuator slit was aligned with the suction surface of the faceplate. Again, the data collapses on to a single curve showing that the mixing enhancement using the synthetic jet actuator is a function of $\beta_{\tau}$ and $C_{\mu, \tau}$.

The actuated wall jet experiment demonstrates that the synthetic jet actuator acting over the clearance region can not only reduce the leakage flow rate as demonstrated by Kang et al. [A.5] but also enhance the mixing of the wall jet increasing the momentum thickness up to about seven times the baseline at $x / \tau=5$. It also shows that there exists a clear frequency dependence of the mixing enhancement and a set of non-dimensional parameters $\left(\beta_{\tau}\right.$ and $C_{\mu, \tau}$ ) characterizing it.

\section{A.5 SUMMARY AND CONCLUSIONS}

The NSJ actuation has been applied to the clearance gap region of the two-dimensional leakage jet to study its effect on the mixing rate of the wall jet. In summary:

- The mixing rate of the wall jet can be promoted significantly - up to seven times increase in the momentum thickness of the shear layer measured five-clearance downstream of the gap - with the use of NSJ acting over the clearance gap region. 
- The mixing enhancement is sensitive to the reduced frequency of the actuation, which scales with the magnitude of the leakage velocity and the tip clearance size.

- The mixing enhancement is also dependent on the momentum coefficient of NSJ.

However, the tip clearance flow in compressor blade passage rolls up into a vortex resulting in a different flow mechanism from the above described two-dimensional wall jet as discussed in Chapter 5 .

\section{REFERENCES}

[A.1] Drazin, P. G. and Reid, W. H., "Hydrodynamic Stability", Cambridge University Press, 1981.

[A.2] Schober, M., Grewe, F., and Fernholz, H. H., "Turbulence Control in Wall Jets: a Visualisation Study", International J. Fluid Dynamics, vol.3, Article l, 1999.

[A.3] Michalke, A., "On Spatially Growing Disturbances in an Inviscid Shear Layer", J. Fluid Mech., 1965, Vol. 23, pp.521-544.

[A.4] Fiedler, H. E. and Mensing, P., "The Plane Turbulent Shear Layer with Periodic Excitation", J. Fluid Mech., 1985, Vol. 150, pp.281-309.

[A.5] Kang, E., Breuer, K. S., and Tan, C. S., "Control of Leakage Flows Using Periodic Excitations", AIAA 2000$2232,2000$.

[A.6] Katz, Y., Horev, E., and Wygnanski, I., "The Forced Turbulent Wall Jet”, J. Fluid Mech., 1992, Vol. 242, pp.577-609.

[A.7] Oster, D. and Wygnanski, I., "The Forced Mixing Layer Between Parallel Streams”, J. Fluid Mech., 1982, Vol. 123, pp.91-130. 